
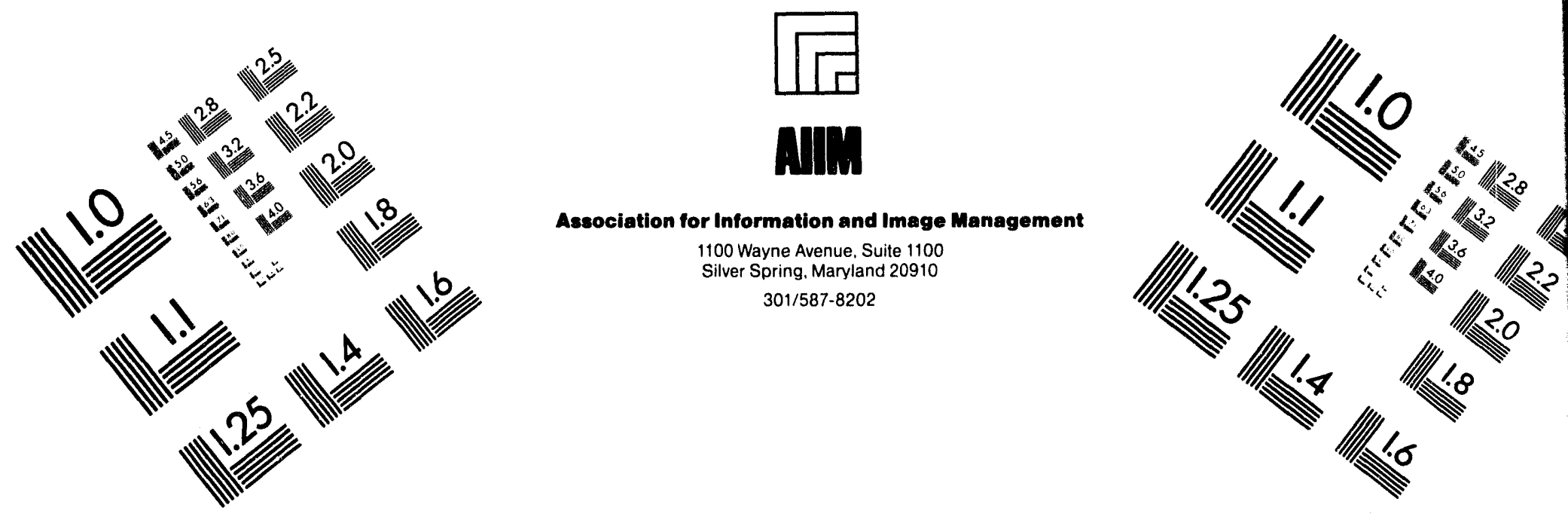

Centimeter

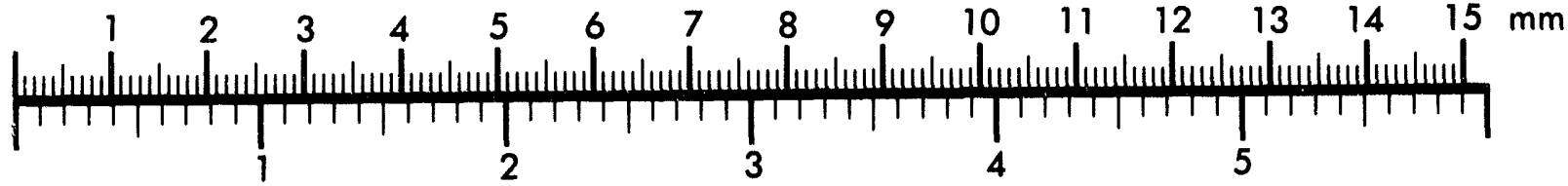
Inches
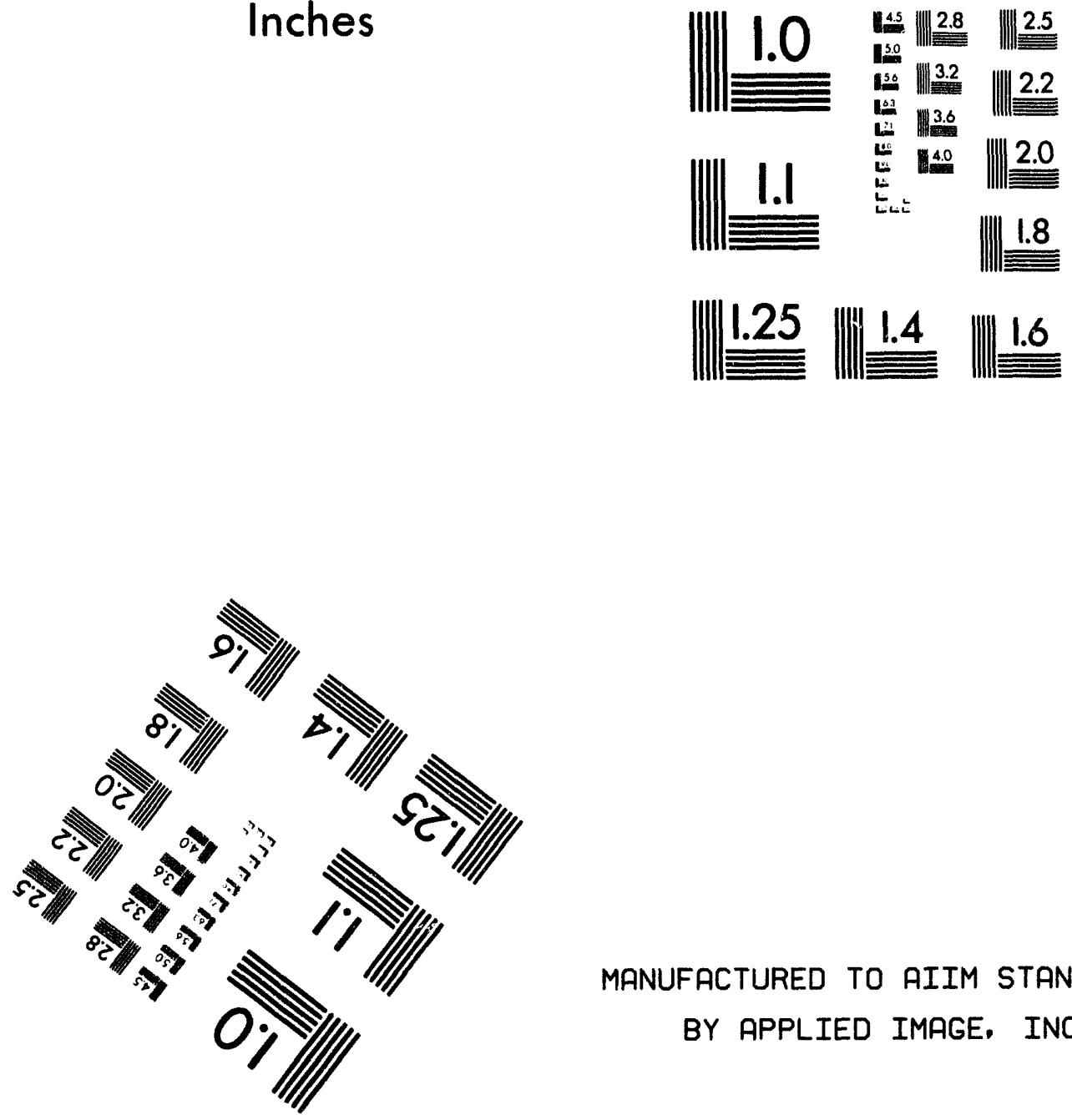

MANUFACTURED TO AIIM STANDARDS

BY APPLIED IMAGE, INC.

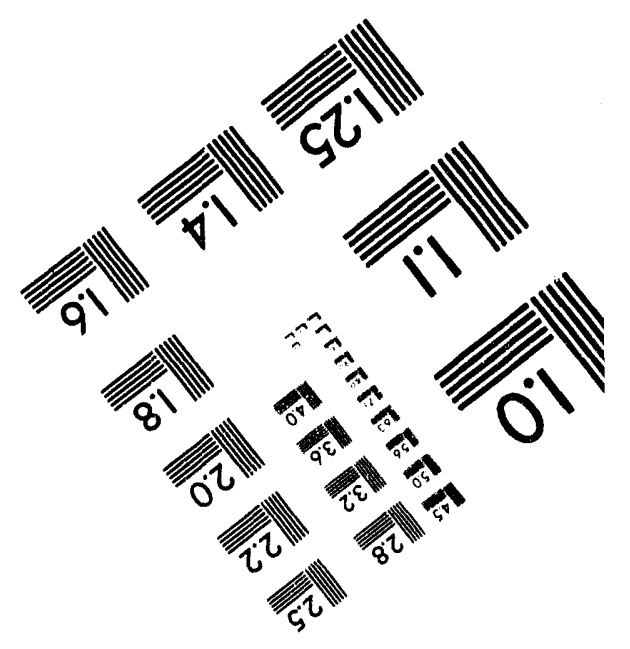



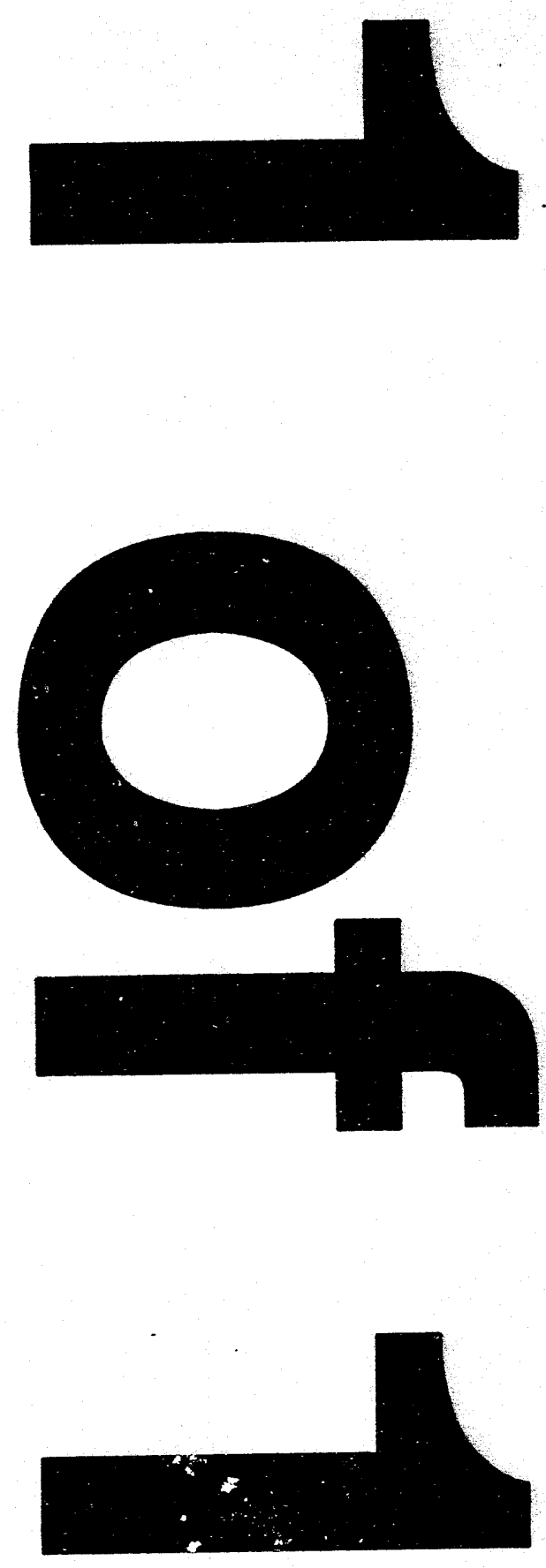


\section{Nuclear Reactors Built, Being Built, or Planned 1993}

Prepared for:

\section{U.S. DEPARTMENT OF ENERGY \\ Director, Office of Nuclear Energy}

Washington, D.C. 20585

Prepared by:

Office of Scientific and

Technical Information 


\section{Preface}

Nuclear Reactors Built, Being Built, or Planned contains unclassified information about facilities built, being built, or planned in the United States for domestic use or export as of December 31, 1993. The Office of Scientific and Technical Information, U.S. Department of Energy, gathers this information annually from Washington headquarters and field offices of DOE; from the U.S. Nuclear Regulatory Commission (NRC); from the U.S. reactor manufacturers who are the principal nuclear contractors for foreign reactor locations; from U.S. and foreign embassies; and from foreign governmental nuclear departments.

The book consists of three divisions, as follows:

- a commercial reactor locator map and tables of the characteristic and statistical data that follow; a table of abbreviations;

- tables of data for reactors operating, being built, or planned; and

- tables of data for reactors that have been shut down permanently or dismantled.

The reactors are subdivided into the following parts: Civilian, Production, Military, Export, and Critical Assembly. Export reactor refers to a reactor for which the principal nuclear contractor is a U.S. company-working either independently or in cooperation with a foreign company (Part IV). Critical assembly refers to an assembly of fuel and moderator that requires an external source of neutrons to initiate and maintain fission. A critical assembly is used for experimental measurements (Part V).

Various classes of reactors within these parts are defined as follows:

Central-Station Electric Power Plant: A nuclear power facility designed and constructed for operation on a utility system (Part I, Section 1A).

Dual-Purpose Plant: A nuclear power facility designed, constructed, and operated for more than one primary purpose, for example, the production of nuclear materials and the generation of electricity or the use of reactor thermal energy for electrical generation and process-heat applications including desalting (Part I, Section 1B).

Experimental Power Reactor: A facility designed, engineered, constructed, and operated to test the technical feasibility of a concept or to provide the technical basis for a similar type nuclear power plant in a larger size. Design flexibility permits changes to prove various aspects of reactor technology including fuel, components, and configurations. Power-conversion equipment may or may not be included as part of the facility (Part I, Section 2).

General Irradiation Test Reactor: A reactor having (1) a thermal power level exceeding $10 \mathrm{MW}$; (2) test loops or experimental facilities within, or in proximity to, the core; and (3) the use of nuclear radiation for testing the life or performance of reactor components as its major function (Part I, Section 3A; Part IV, Section 2A).

High-Power Research and Test Reactor: A reactor having a relatively high thermal power level (5MW or greater) but not classed as a general irradiation test reactor (Part I, Section 3B).

Safety-Research and Test Reactor: A reactor associated with a nuclear safety research or engineering-scale test program conducted for the purpose of developing basic design information or demonstrating safety characteristics of terrestrial and aerospace nuclear reactor systems (Part I, Section 3C).

General Research Reactor: A reactor-excluding that located at a university - whose nuclear radiations are used primarily as a research tool for basic or applied research and whose thermal power level is $10 \mathrm{MW}$ or less. It may include facilities for testing reactor materials (Part I, Section 3D; Part IV, Section 2B). Also included are Research Reactors (Part III, Section 3B).

University Research and Teaching Reactor: A reactor located at a university and usually operated for the primary purpose of training in the operation and utilization of reactors and for instruction in reactor theory and performance (Part I, Section 3E; Part IV, Section 2C).

Reactors are further grouped according to status:

Reactors are listed as operable under the following circumstances:

1. Reactors regulated by the NRC

- when an operating license is issued.

- when a reactor is temporarily shut down because of technical reasons, modifications, or refueling.

2. Federal Government reactors

- when criticality is achieved.

- when a reactor is temporarily shut down for safety improvements.

3. Reactors for export

- when criticality is achieved.

Reactors are listed as being built under the following circumstances:

1. Reactors regulated by NRC

- when a construction permit is issued.

- when limited work authorization is issued.

2. Federal Government reactors

- when ground is broken.

- when components are ordered.

- when a construction contract is awarded. 
3. Reactors for export

- when an application for an export license is received by NRC.

- when reliable information is received relating to the fabrication of reactor components.

Reactors are listed as planned und r the following circumstances:

1. Reactors regulated by NRC

- when a public announcement that includes the principal vendor supplier is made by the sponsoring organization.

- when an application for a construction permit is received by NRC.

2. Federal Government reactors

- when a public announcement is made by the agency involved.

- when the project is otherwise appropriately authorized.

3. Reactors for export

- when a public announcement that includes principal contractor and reactor type is made.

- when NRC receives information that a U.S. reactor manufacturer is proceeding with preconstruction design and development on the basis of a letter of intent.

Reactors are considered to be shutdown or dismantled under the following circumstances:

1. Reactors regulated by NRC

- when the licensee has applied to the Commission for authority to surrender a license voluntarily and to dismantle the facility and dispose of its component parts. A reactor shut down because of technical problems, modifications, or refueling, continues to be listed as operable.

2. Federal Government reactors

- when the facility has ceased operation and the agency has declared officially that the agency does not intend to operate the reactor further. However, within this category, a few reactors are identified as being in standby mode, the condition in which documentary authorization exists to maintain the reactor for possible future operation.
3. Reactors for export

- when the plant is officially declared shut down by the owner and taken out of operation permanently.

Table 2 presents a statistical summary of reactors, other than critical assemblies, in each class and status. Shutdown and dismantled reactors are included since such facilities have made significant contributions to reactor technology.

The reactor tables have the following column headings:

- Location. The city and state or country where located originally. For a portable facility or one that has been relocated, the most recent location is given.

- Principal nuclear contractor, operator, designer, shipbuilder. The abbreviations used in this column are spelled out in Table 3, which appears just before the reactor tables.

- Type. Entries in this column are based on coolant, moderator, and neutron energy.

- Power. MDcapacity [MW(e)] is the maximum dependable capacity (net electrical output to grid) for plants having an operating history. Otherwise, it is the design capacity. Licensed power and authorized power are given where appropriate.

- Designation. The common name, abbreviation or acronym used for the facility. For the naval reactors, it is the hull number.

- Date columns. The initial criticality date, year of operation, and year of shutdown are given as appropriate.

Nuclear Reactors Built, Being Built, or Planned (DOE/ OSTI--8200-R57) is sponsored by the DOE Office of Nuclear Energy, LaRue E. Moxley, Program Officer.

The participation and assistance of many individuals, agencies, and companies in providing data and updating the entries in this revision are gratefully acknowledged. Comments and suggestions about this publication are welcome. To ensure that the wide range of information included in this publication will continue to be timely and accurate, please direct any information related to updating the items to William F. Simpson, Jr., Office of Scientific and Technical Information, Scientific and Technical Publications Branch, P.O. Box 62, Oak Ridge, TN 37831; (615) 576-1228. Questions of a technical nature should be addressed to Lamar Cason at the same address. 


\section{CONTENTS}

Preface

Map: Commercial Nuclear Power Reactors in the United States

viii

Table 1. Commercial Nuclear Power Reactors in the United States as of 31 Dec 1993

ix

Table 2. Statistical Summary of Nuclear Reactors as of 31 Dec $1993 \quad$ XV

Table 3. Abbreviations of Contractors, Designers, Shipbuiiders, and Facility Operators

xvi

\section{REACTORS AND FACILITIES}

\section{OPERABLE, BEING BUILT, OR PLANNED}

\section{Part I. Civilian Reactors (Domestic)}

1. Power Reactors

A. Central-Station Electric Power Plants

B. Dual-Purpose Plants (No reactors currently in this category)

C. Propulsion (Maritime) (No reactors currently in this category)

2. Experimental Power-Reactor Systems
A. Electric-Power Systems
B. Space Nuclear Auxiliary Power (SNAP) (No reactors currently in this category)

C. Space Propulsion (No reactors currently in this category)

3. Test, Research, and University Reactors
A. General Irradiation Test
B. High-Power Research and Test
C. Safety Research and Test
D. General Rescarch
E. University Research and Teaching

Part II. Production Reactors

1. Materials Production

2. Process Development (No reactors currently in this category) 


\section{CONTENTS (Continued)}

Part III. Military Reactors

1. Defense Power-Reactor Applications

A. Remote Installations (No reactors currently in this category) 12

B. Propulsion (Naval) 12

2. Developmental Power 15

A. Electric-Power Experiments and Prototypes (No reactors currently in this calegory)

B. Propulsion Experiments and Prototypes 15

3. Test and Rescarch

A. Test (No reactors currently in this category)

B. Rescarch

\section{Part IV. Export Reactors}

1. Power Reactors 16

A. Central-Station Electric Power Plants 16

B. Propulsion (No reactors currently in this category) $\quad 20$

2. Test, Rescarch, and Teaching 20

A. General Irradiation Test $\quad 20$

B. General Research $\quad 20$

C. University Research and Teaching 22

Part V. Critical Assemblies

1. Civilian 23

2. Military 24

\section{REACTORS AND FACILITIES}

SHUTDOWN OR DISMANTLED

Part I. Civillan Reactors (Domestic)

1. Power Reactors

A. Central-Station Electric Power Plants 25

B. Dual-Purpose Plants 26

C. Propulsion (Maritime) 27

2. Experimental Power-Reactor Systems $\quad 27$

A. Electric-Power Systems $\quad 27$

B. Space Nuclear Auxiliary Power (SNAP) 29

C. Space Propulsion $\quad 30$

3. Test, Research, and University Reactors 31

A. General Irradiation Test 31

B. High-Power Research and Test

C. Safcty Research and Test 32

D. General Research 33

E. University Research and Teaching 37 


\section{CONTENTS (Continued)}

Part II. Production Reactors

1. Materials Production

2. Process Development

Part III. Miltary Reactors

1. Defense Power-Reactor Applications $\quad 40$

A. Remote Installations $\quad 40$

B. Propulsion (Naval)

2. Developmental Power $\quad 43$

A. Electric-Power Experiments and Prototypes $\quad 43$

B. Propulsion Experiments and Prototypes 43

3. Test and Research
A. Test

B. Research

\section{Part IV. Export Reactors}

1. Power Reactors

A. Central-Station Electric Power Plants 44

B. Propulsion $\quad 44$

2. Test, Research, and Teaching 45

A. General Irradiation Test (No reactors currently in this category)

B. General Research 45

C. University Research and Teaching 45

Part V. Critical Assemblles

$\begin{array}{ll}\text { 1. Civilian } & 46\end{array}$

2. Military 46

REACTOR INDEX 


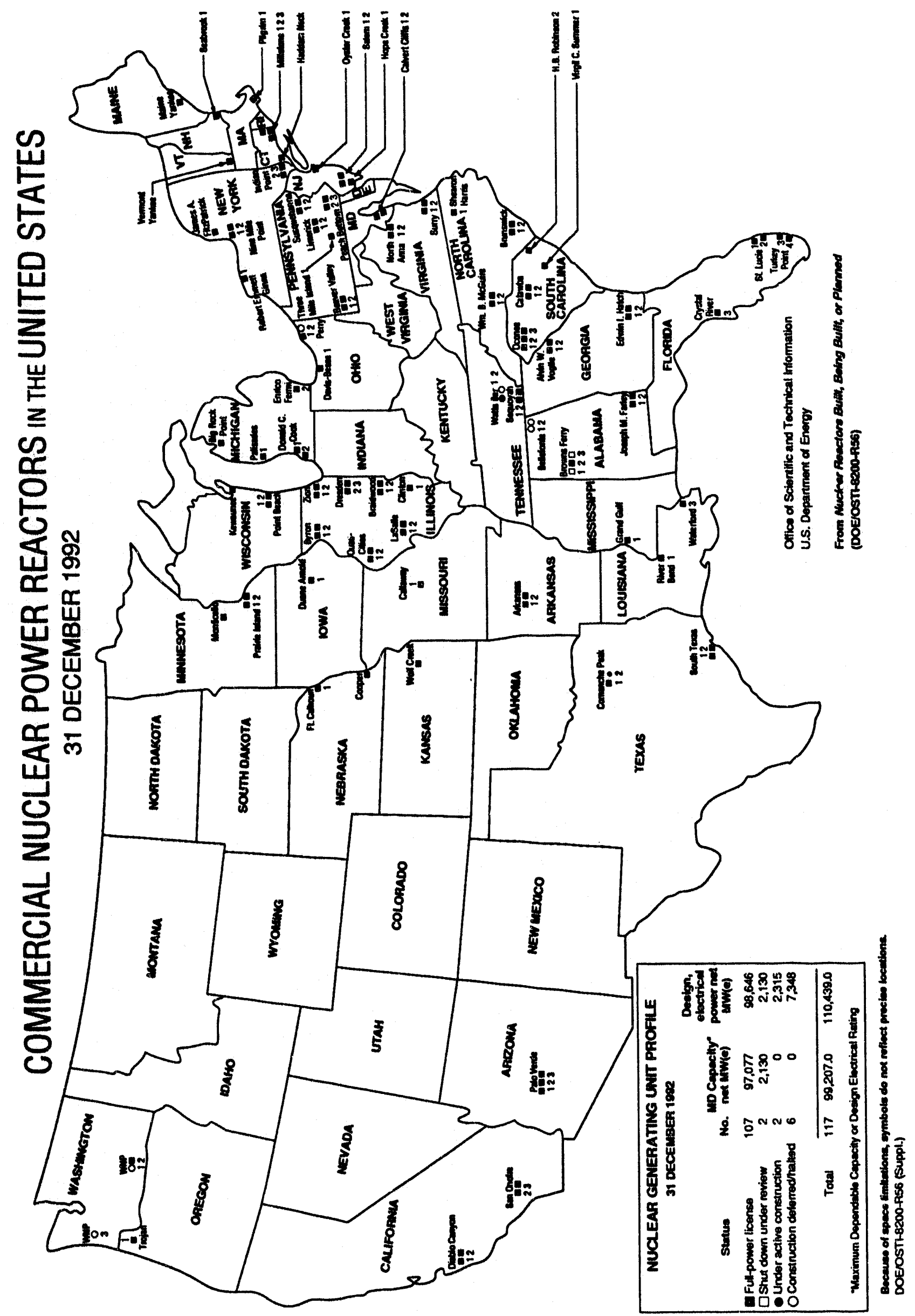

viii 
TABLE 1

COMMERCIAL NUCLEAR POWER REACTORS IN THE UNITEC STATES AS OF 31 DEC 1993

SITE

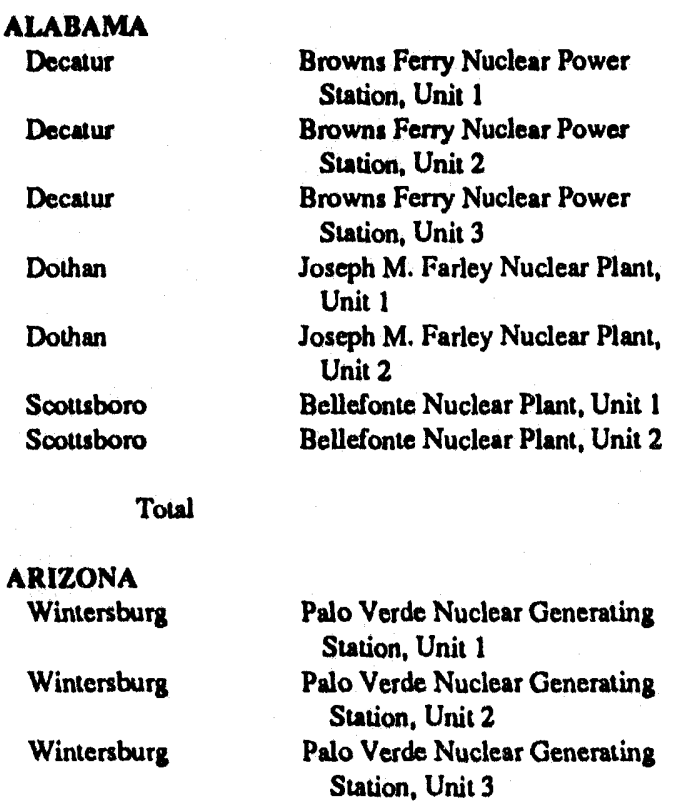

Total

ARKANSAS

Russellville

Russellville

Total

\section{CALIFORNIA}

Diablo Canyon

Diablo Canyon

San Clemente

San Clemente

Total

CONNECTICUT

Haddam Neck

Waterford

Waterford

Waterford

Total
Arkansas Nuclear One, Unit 1 Arkansas Nuclear One, Unit 2

Diablo Canyon Nuclear Power Plant, Unit 1

Diablo Canyon Nuclear Power Plant, Unit 2

San Onofre Nuclear Generating Stution, Unit 2

San Onofre Nuclear Generating Station, Unit 3

FPL

1,221

1,270

FPL

1.221

1,270

FPL

1,304

1,270

3,746

3,810

FPL

\begin{tabular}{cr}
836 & 850 \\
858 & 912 \\
\hline 1,694 & 1,762
\end{tabular}

FPL $\quad 1,073$

1,086

FPL $\quad 1,087$

1,119

FPL $\quad 1,070$

1,070

FPL

1,080

1,080

4,310

4,355

Haddam Neck Plant

Millstone Nuclear Power

Station, Unit 1

Millstone Nuclear Power

Station, Unit 2

Millstone Nuclear Power

Station. Unil 3

$\begin{array}{lrr}\text { FPL } & 560 & 582 \\ \text { FPL } & 641 & 660 \\ \text { FPL } & 873 & 870 \\ \text { FPL } & 1,137 & 1,154 \\ & 3,211 & 3,266\end{array}$

LICENSEA

STARTUP

7308

7407

7608

7708

8105

Indof.

Indef.
Arizona Public Service Co. $\quad 8505$

Arizona Public Service Co. $\quad 8604$

Arizona Public Service Co. $\quad 8710$

Entergy Operations Inc.
Connecticut Yankee Atomic $\quad 6707$ Power Co.

Northeast Nuclear Energy Co. $\quad 7010$

Northeast Nuclear Energy Co. $\quad 7510$

Northeast Nuclear Energy Co. $\quad 8601$ 
TABLE 1 (Contlnued)

SITE

plant name

CAPACITY,

DESIGN

ELECTRICAL

RATING,

LORIDA

Florida City

Florida City

Fon Pierce

Fon Pierce

Red Level

Turkey Point Plant, Unit 3

Turkey Point Plant, Unit 4

St. Lucie Plant, Unit 1

St. Lucie Plant, Unit 2

Crystal River Nuclear Plant, Unit 3

STATUS

NET MW(e)

NET MW(e)

LICENSEE

STARTUP

$\begin{array}{lll}\text { FPL } & 666 & 693 \\ \text { FPL } & 666 & 693 \\ \text { FPL } & 839 & 830 \\ \text { FPL } & 839 & 830 \\ \text { FPL } & 821 & 825\end{array}$

Florida Power \& Light Co.

GEORGIA

Baxlcy

Edwin I. Hatch Nuclear Plant,

FPL

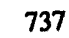

776

Georgia Power Co.

7409

Unit 1

Baxlcy

Edwin I. Hatch Nuclear Plant.

FPL

757

784

Georgia Power Co.

7807

Alvin W. Vogule Nuclear Plant,

FPL

1,158

1,101

Georgia Power Co.

8703

Unit 1

Waynesboro

Alvin W. Vogule Nuclear Plant, Unit 2

FPL

1,157

1,101

Georgia Power Co.

8903

Total

3,809

3,762

\section{ILLINOIS}

Braidwood

Braidwood

Byron

Byron

Clinton

Cordova

Cordova

Morris

Morris

Sineca

Seneca

Zion

Zion

Braidwood Station, Unit !

Braidwood Station, Unit 2

Byron Station, Unit 1

Byron Station, Unit 2

Clinton Power Station, Unit 1

Quad-Cities Station, Unit I

Quad-Cities Station. Unit 2

Dresden Nuclear Power Station, Unit 2

Dresden Nuclear Power Station, Unit 3

La Salle County Station, Unit I

La Salle County Station, Unit 2

Zion Nuclear Plant, Unit 1

Zion Nuclcar Planı, Unit 2

FPL
FPL
FPL
FPL
FPL
FPL
FPL
FPL
FPL
FPL
FPL
FPL
FPL

Total

IOWA

Palo

Total

KANSAS

Burlington

Total

LOUISIANA

St. Francisville

Taft

Wolf Creck Generating Station

\section{Duane Amold Energy Center,} Unit 1

FPL.

1,134

1,170

1.134

1,170

River Bend Station, Unit 1 Waterford Generating Station, Unit 3

515

538

515

538

FPL

FPL

936

\begin{tabular}{rr}
1,120 & 1,120 \\
1,120 & 1,120 \\
1,105 & 1,120 \\
1,105 & 1,120 \\
930 & 933 \\
769 & 789 \\
769 & 789 \\
772 & 794 \\
773 & 794 \\
1,036 & 1,078 \\
1,036 & 1,078 \\
1,040 & 1,040 \\
1,040 & 1,040 \\
\hline 12,615 & 12,815
\end{tabular}

Commonwealth Edison Co. Commonwealth Edison Co. Commonwealth Edison $\mathrm{Co}$. Commonwealth Edison $\mathrm{Co}$. Illinois Power Co.

Commonwealth Edison Co.

Cummonwealth Edison $\mathrm{Co}$.

Cummonwealth Edison Co.

Commonwealth Edison Co.

Commonwealth Edison Co.

Commonwealth Edison Co.

Commonwealth Edison Co.

Commonwealth Edison Co.

8705

8803

8502

8701

8702

7110

7204

7001

7101

8206

8403

7306

7312

Iowa Electric Light \&

Power Co.

Wolf Creck Nuclear Operating Corp.

8505

Gulf States Utilities Co.

Entergy Operations Inc.

8510 


\section{TABLE 1 (Continued)}

SITE

Total

\section{MARYLAND}

Lusby

Lusby

Total

MASSACHUSETTS

Plymouth

Total

MICHIGAN

Big Rock Point

Bridgman

Bridgman

Newport

South Haven

Total

\section{MINNESOTA}

Monticello

Red Wing

Red Wing

Total

\section{MISSISSIPPI}

Pont Gibson

Total

\section{MISSOURI}

Fulton

Tolal

\section{NEBRASKA}

Brownville

Fon Calhoun

Total

Big Rock Point Nuclear Plant Plant, Unit 1 Plant, Unit 2

Enrico Fermi Alomic Power

Plant, Unit 2 Plant$$
\text { Plant, Unit } 1
$$
Plant, Unit 2

Grand Gulf Nuclear Station, Unit 1

Callaway Plant, Unit 1 For Calhoun Station, Unit 1
Plant Name

Maine Yankee Alomic Power Plant

\section{Calven Cliffs Nuclear Power Plant, Unit 1 \\ Calvet Cliffs Nuclear Power Plant, Unit 2}

Pilgrim Nuclear Power Station, Unit 1

FPL CAPACITY, DESIGN ELECTRICAL RATING, STATUS NET MW(e) NET MW(e) LICENSEE

STARTUP

FPL

\begin{tabular}{ll}
860 & 870 \\
\hline 860 & 870
\end{tabular}

FPL

830

845

830

845

FPL

1,660

1,690

670

655

Boston Edison Co.

7206

Maine Yankee Alomic Power Co.

Baltimore Gas \& Electric Co.

7410

Baltimore Gas \& Electric Co.

7611

$670 \quad 655$

FPL. 67 1,000

72 Donald C. Cook Nuclear Power

Donald C. Cook Nuclear Power

Palisades Nuclear Plant, Unit 1

FPL

1,060

1,020

FPL

1,060

1,090

FPL

1,085

1,116

FPL

\begin{tabular}{lr}
730 & 805 \\
\hline 3,942 & 4,103
\end{tabular}

FPL 536 545 Monticello Nuclear Generating

FPL

513

$$
530
$$

Prairie Island Nuclear Generating

FPL

512

530

1,561

1.605

FPL

1,143

1,250

1,143

1,250

Cooper Nuclear Station
Consumers Power Co. $\quad 6209$ Indiana and Michigan 7501 Electric Co.

Indiana and Michigan $\quad 7803$

Electric Co.

Detroit Edison Co. $\quad 8506$

Consumers Power Co. 7105

Nonhem States Power Co. $\quad 7012$

Northem States Power Co. $\quad 7312$

Northem States Power Co. $\quad 7412$

Entergy Operations Inc.

8208

Union Electric Co.

8410

Nebraska Public Power District $\quad 7402$ Omaha Public Power District $\quad 7308$
FPL

\begin{tabular}{ll}
$1,120 \quad 1,171$ \\
\hline 1,120 & 1,171
\end{tabular}

FPL

FPL

\begin{tabular}{ll}
764 & 778 \\
478 & 478 \\
\hline 1,242 & 1,256
\end{tabular}


TABLE 1 (Continued)

STTE

\section{NEW HAMPSHIRE}

Seabrook

Total

\section{NEW JERSEY}

Salem

Salem

Salem

Toms River

Total

\section{NEW YORK}

Buchanan

Buchanan

Ontario

Scriba

Scriba

Scribe

Total

\section{NORTH CAROLINA}

Boneal

Cowans Fond Dam

Shearon Harris Nuclear Power

Plans, Unit 1

Hope Creek Nuclear Generating Station, Unit 1

Salem Nuclear Generating

Sution, Unit 1

Salem Nuclear Generating

Station, Unit 2

Oyster Creek Nuclear Power

Plant, Unit I

\section{Indian Point Station, Unit 2 \\ Indien Point Sution, Unit 3 \\ Robert Emmeu Ginna Nuclear \\ Power Plant, Unit ! \\ Jamea A. FitzPatrick Nuclear \\ Power Plant \\ Nine Mile Point Nuclear \\ Station, Unit 1 \\ Nine Mile Point Nuclear \\ Station, Unir 2}

1,031

1,067

FPL

1,106

1,115

FPL

1,106

1,115

FPL

610

650

3,853

3,947

$\begin{array}{lll}\text { FPL } & 951 & 986\end{array}$

FPL $\quad 965 \quad 965$

$\begin{array}{lll}\text { FPL } & 470 & 470\end{array}$

FP

780

816

FPL

565

625

FPL

994

1,062

4,725

4,924

FPL

860

900

FPL

1,129

1,180

Station, Unit I

Cowans Ford Dam

William B. McGuire Nuclear Station, Unit 2

Southpon

Southport

Brunswick Steam Electric Plant, Unit 1

Brunswick Steam Electric Plant, Unit 2

FPL

1.129

1,180

FPL

767

821

FPL

754

821

4,639

4,902

OHIO

Oak Harbor

Perry

Perry
Davis-Bease Nuclear Power Station, Unit 1

Perry Nuclear Power Plant, Unit 1

Perry Nuclear Power Plant. Unit 2
FPL

FPL

$\mathrm{CDH}$
877

1,166

1,191

1,205
LICENSEE

STARTUP

8906

North Allantic Energy Service Corp.

Public Service Electric \&

8606

Gas Co.

Public Service Electric \&

7612

Gas Co.

Public Service Electric \&

8008

Gas Co.

GPU Nuclear Corp.

6905

Consolidated Edison Co. of

7305

New York, Ine.

Now York Power Authority $\quad 7604$

Rochester Gas \& Electric Corp. 6911

New York Power Authority 7411

Nisgara Mohawk Power Corp. $\quad 6909$

Niagan Mohawk Power Corp. 8705

Carolina Power \& Light Co. $\quad 8701$

Duke Power Co.

8108

Duke Power Co.

8305

Carolina Power \& Light Co.

7610

Carolina Power \& Light Co.

7503

$2,043 \quad 3,302$

7708

8606

Indef. 
TABLE 1 (Continued)

STTE

PLANT NAME

Suequehanna Sieam Electric

Station, Unit 1

Susquehanna Steam Electric

Station, Unil 2

Lencaster

Lancaster

Middletown

Poustown

Potetsown

Shippinepon

Shippingpon

Total

SOUTH CAROLINA

Hartsville

Jenkinsville

Lake Wylie

Lake Wylie

Seneca

Seneca

Senece

Total

TENNESSEE

Daisy

Daisy

Spring City

Spring City

Total

\section{TEXAS}

Bay Cily

Bay City

Gien Rose

Glen Rose

Total

\section{VERMONT}

Vemon

Peach Bottom Alomic Power Station, Unit 2

Peach Bottom Alamic Power Station, Unit 3

Three Mile Islend Nuclear Station, Unil 1

Limerick Generating Station. Unit 1

Limerick Generating Station. Unit 2

Beaver Valley Power Sution, Unit 1

Beaver Valley Power Sution, Unit 2

H.B. Robinson Plant, Unit 2

Virgil C. Summer Nuclear

Station, Unit 1

Station, Unit 2
Catawba Nuclear Station, Unit 1

Catawba Nuclear Station, Unit 2

Oconee Nuclear Station, Unit 1

Oconee Nuclear Station, Unit 2

Oconee Nuclear Station, Unit 3

FPL
FPL
FPL
FPL
FPL
FPL
FPL

FPL $\quad 1,040$

1,050

FPL $\quad 1,044$

1,050

FPL 1,055

1,065

FPL. $\quad 1,035$

1,065

FPL $\quad 786$

819

FPL $\quad 1,055$

1,055

FPL $\quad 1,055$

1,055

FPL $\quad 810$

835

FPL

820

836

$8,700 \quad 8,830$

$683 \quad 700$

885900

\begin{tabular}{rr}
1,129 & 1,145 \\
1,129 & 1,145 \\
846 & 886 \\
846 & 886 \\
846 & 886 \\
\hline 6,364 & 6,548
\end{tabular}

Sequoyah Nuclear Plant, Unit 1 Sequoyah Nuclear Plant, Unit 2 Watts Bar Nuclear Plant, Unit 1 Watts Bar Nuclear Plant, Unit 2

FPL
FPL
UC
CDH

\begin{tabular}{ll}
1,122 & 1,148 \\
1,122 & 1,148 \\
& 1,165 \\
& 1,165 \\
\hline 2,244 & 4,626
\end{tabular}

FPL $\quad 1,251 \quad 1,251$

$\begin{array}{lll}\text { FPL } & 1,251 & 1,251\end{array}$

$\begin{array}{lll}\text { FPL } & 1,150 & 1,150\end{array}$

FPL.

1,150

1,150

Comanche Peak Steam Electric

\section{4,802}

4,802

Vermoni Yankee Nuclear Power Station

FPL

\begin{tabular}{cc}
504 & 514 \\
\hline 504 & 514
\end{tabular}

LICENSEE

STARTUP

8209

8405

Pennsylvania Power \&

Light Co.

Philadelphia Electric Co.

7309

Philadelphia Electric Co.

7408

7406

8412

8908

7605

8708

Duquesne Light Co.

7009

8210

8501

8605

7304

7311

7409
8007

8111

Tennessee Valley Authority

Tennessee Valley Authority

Tennessee Valley Authority

Indef.

Houston Lighting \& Power Co.

Texas Utilities Generating Co.

9308

\footnotetext{
Vermont Yankee Nuclear Power Corp.
} 


\section{TABLE 1 (Continued)}

SITE

PLANT NAME

Surry Power Station, Unit 2

North Anna Power Station, Unit 1

Nonth Anna Power Station, Unit 2

Total

\section{WASHINGTON}

Richland

Richland

Satsop

Total

WISCONSIN

Cartion

Two Creeks

Two Crecks

Total

U.S. Total

FPL, Full-Power License

UC. Under Active Construction

Washington Nuclear Project,
Unit 1
Washington Nuclear Project,
Unit 2
Washington Nuclear Project,
Unit 3
Kewaunee Nuclear Power Plant
Point Beach Nuclear Plant,
Unit 1
Point Beach Nuclear Y tant,
Unit 2

Surry Power Station, Unit I

CDH

1,266

FPL

\begin{tabular}{lll} 
& & 1,266 \\
PL & 1,086 & 1,100 \\
& & 1,242 \\
& & \\
\hline & 1,086 & 3,608
\end{tabular}

1,100

$\mathrm{CDH}$

CAPACITY,

DESIGN

ELECTRICAL

RATING,

NET MW(e)

LICENSEE

STARTUP

\begin{tabular}{lll} 
FPL & 781 & 788 \\
FPL & 781 & 788 \\
FPL & 900 & 907 \\
FPL & 887 & 907 \\
\hline & 3,349 & 3,390
\end{tabular}

FPL

511

535

FPL

485

497

FPL

485

497

Washington Public Power

Supply System

Washington Public Power $\quad 8401$

Supply System

Washington Public Power Indef.

Supply System

Wisconsin Public Service Corp. $\quad 7403$

Wisconsin Electric Power Co. $\quad 7011$

Wisconsin Electric Power Co. $\quad 7205$

\begin{tabular}{ll}
\hline 1,481 & 1,529 \\
\hline 95,713 & 109,382
\end{tabular}

CDH, Construction Deferred/Halted

SDUR, Shut Down Under Review 
TABLE 2

\section{STATISTICAL SUMMARY OF NUCLEAR REACTORS AS OF 31 DEC 1993}

\begin{tabular}{|c|c|c|c|c|c|}
\hline & Operable & $\begin{array}{l}\text { Being } \\
\text { built }\end{array}$ & Planned & Shutdown & Totals \\
\hline \multicolumn{6}{|l|}{ U.S. REACTORS } \\
\hline \multicolumn{6}{|l|}{$\begin{array}{l}\text { CIVILIAN REACTORS (DOMESTIC) } \\
\text { Power Reactors }\end{array}$} \\
\hline $\begin{array}{l}\text { Central-Station Electric Power Plants } \\
\text { Dual-Purpose Plants } \\
\text { Propulsion (Maritime) } \\
\text { Experimental Power-Reactor Systems }\end{array}$ & 109 & 7 & & $\begin{array}{r}20 \\
1 \\
1\end{array}$ & $\begin{array}{r}136 \\
1 \\
1\end{array}$ \\
\hline $\begin{array}{l}\text { Electric-Power Systems } \\
\text { Spece Nuclear Auxiliary Power (SNAP) } \\
\text { Space Propulsion (Rover) } \\
\text { Test, Research, and University Reactors }\end{array}$ & 1 & & & $\begin{array}{r}23 \\
9 \\
21\end{array}$ & $\begin{array}{r}24 \\
9 \\
21\end{array}$ \\
\hline General Irradiation Test & 1 & & 1 & 6 & 8 \\
\hline High-Power Research and Test & 5 & & & 7 & 12 \\
\hline Safety-Research and Test & 1 & & & 10 & 11 \\
\hline General Research & 14 & & 1 & 56 & 71 \\
\hline $\begin{array}{l}\text { University Research and Teaching } \\
\text { PRODUCTION REACTORS }\end{array}$ & 34 & & 1 & 31 & 66 \\
\hline $\begin{array}{l}\text { Materials Production } \\
\text { Process Development } \\
\text { MILTARY REACTORS }\end{array}$ & 1 & & & $\begin{array}{c}12 \\
5\end{array}$ & $\begin{array}{r}13 \\
5\end{array}$ \\
\hline $\begin{array}{l}\text { Defense Power-Reactor Applications } \\
\text { Remote Installations }\end{array}$ & & & & 6 & 6 \\
\hline $\begin{array}{l}\text { Propulsion (Naval) } \\
\text { Devclopmental Power }\end{array}$ & 124 & 15 & & 72 & 211 \\
\hline $\begin{array}{l}\text { Electric-Power Experiments and Protolypes } \\
\text { Propulsion Experiments and Protokypes } \\
\text { Test and Research }\end{array}$ & 5 & & & $\begin{array}{c}3 \\
10\end{array}$ & $\begin{array}{r}3 \\
15\end{array}$ \\
\hline $\begin{array}{l}\text { Test } \\
\text { Research }\end{array}$ & 4 & & & $\begin{array}{l}3 \\
6\end{array}$ & $\begin{array}{r}3 \\
10\end{array}$ \\
\hline \multicolumn{6}{|l|}{ EXPORT REACTORS } \\
\hline POWER REACTORS & & & & & \\
\hline $\begin{array}{l}\text { Central-Station Electric Power Plants } \\
\text { Propulsion } \\
\text { TEST, RESEARCH, AND TEACHING }\end{array}$ & so & 11 & 4 & $\begin{array}{l}5 \\
1\end{array}$ & $\begin{array}{r}70 \\
1\end{array}$ \\
\hline General Irradiation Test & 7 & & & & 7 \\
\hline $\begin{array}{l}\text { General Research } \\
\text { University Research and Teaching }\end{array}$ & $\begin{array}{l}27 \\
19\end{array}$ & 1 & 1 & $\begin{array}{r}10 \\
6\end{array}$ & $\begin{array}{l}39 \\
25\end{array}$ \\
\hline Totals & 402 & $\overline{34}$ & $\overline{8}$ & $\overline{324}$ & 768 \\
\hline
\end{tabular}


TABLE 3

\section{ABBREVIATIONS OF CONTRACTORS, DESIGNERS, SHIPBUILDERS, AND FACILITY OPERATORS}

The definitions of the following abbreviations that have been used in this volume contain references to current and historical corporate and government structure.

\begin{tabular}{|c|c|}
\hline $\begin{array}{l}\text { AC } \\
\text { ACEC }\end{array}$ & $\begin{array}{l}\text { Allis-Chabmens Mf:. Co. } \\
\text { Aleliers de Consuruction Electriques de Charleroi S.A. (Belgium) }\end{array}$ \\
\hline ACF & ACF Industries, Inc. (reactor sctivities abmendoned by AC) \\
\hline AEC & Alomic Energy Commission, a predecessor of the Depertment of Energy \\
\hline AG & Aerojet-Genenal Corporation \\
\hline AGN & Aerojet-General Nuclocnics, formenty a subsidiary and then a division of \\
\hline AI & $\begin{array}{l}\text { Aerojet-Generil Corporation } \\
\text { Alomics Imtemational, a division of Rockwell Intemational }\end{array}$ \\
\hline Aloo & Aloo Products, Inc. (reactor activities sbsorbed by AC) \\
\hline AMF & AMF Atomics, Inc, a division of Americen Machine \& Foundry Ca. \\
\hline ANL & Argonne Nationsl Laborntory \\
\hline ANPD & $\begin{array}{l}\text { Aircraft Nuclear Propolsion Department, General Electric Company } \\
\text { (name changed to Flight Propulsion Laboratory Deparment) }\end{array}$ \\
\hline AR & American Radintor \\
\hline AS Inc. & American Simdard Inc. \\
\hline AU & Associated Universinies, Inc. (Brookhaven National Laboratory) \\
\hline BAC & Bendix Avizion Corp. \\
\hline Bethlehem & $\begin{array}{l}\text { Shipbuilding Division, Bethlehem Steel Co. (now Quincy Division, } \\
\text { General Dynamics Corp.) }\end{array}$ \\
\hline Betris & Benis Atomic Power Laboratory \\
\hline Blaw-Knox & Blaw-Knox Co. \\
\hline B\&R & Bums \& Roe, Inc. \\
\hline B\&W & Baboock \& Wilcox Co. \\
\hline BNL & Brookthaven National Laborationy \\
\hline CL & Clinton Laboratory of the Manhakan Engineer District \\
\hline Comb. & Combustion Engineering, Inc. \\
\hline Convair & Convair Division, General Dynamics Corp. \\
\hline Cook & Nucledyne $C_{0 .,}$ a division of Cook Electric Company \\
\hline $\mathbf{C W}$ & Curtiss-Wright Corporation \\
\hline Daystrom & Daystrom, Inc. \\
\hline DNA & Defense Nuclear Agency, Depanment of Defense \\
\hline DOD & Department of Defense \\
\hline DOE & Department of Energy \\
\hline Du Pont & E.I. Du Pont de Nemours \& Company, Inc. \\
\hline EG\&G-ID & EG\&G Idaho, Inc. (a division of EG\&G, Inc) \\
\hline Electric Boat & Electric Boat Division, General Dynamics Corp. \\
\hline Fluor & The Fluor Corporation, Led. \\
\hline Fram. & Framatome \\
\hline FW & Foster Wheeler \\
\hline
\end{tabular}

\begin{tabular}{|c|c|}
\hline GA & General Alomics Tectmologies \\
\hline GD (Quincy) & Quincy Division, General Dymmics Corp. \\
\hline GE & General Electric Company \\
\hline GNEC & $\begin{array}{l}\text { General Nuclear Engineering Corp. (became a division of Combustion Engineering. } \\
\text { Inc., in 1964) }\end{array}$ \\
\hline IC & Intemuclear $\mathrm{Co}$. \\
\hline INC & Idaho Nuclear Corporation \\
\hline INEL & Idaho Nacional Engineering Laboratory \\
\hline Ingalls & Ingalls Shipbuilding Corp. \\
\hline Kaman & Kaman Nuclear, a division of Kaman Aircraft Corp. \\
\hline KAPL & Knolls Atamic Power Laboratory \\
\hline KE & Kaiser Engineers, a division of Henry J. Kriser Co. \\
\hline LANL & Los Alamos National Laboratory \\
\hline LLNL & Lawrence Livermore National Laboratory \\
\hline $\begin{array}{l}\text { Lockbeed } \\
\text { Mare Island }\end{array}$ & $\begin{array}{l}\text { Lockheed Aircraft Corp. } \\
\text { Mare Island Naval Shipyard }\end{array}$ \\
\hline $\begin{array}{l}\text { Mare isiand } \\
\text { Martin }\end{array}$ & Martin Marieta Corp. \\
\hline Maron & Maxon Construction Ca. \\
\hline Ma. Lab & Metallurgical Laboratory of the Manhauan Engineer District \\
\hline NASA & National Aeronautics and Space Administration \\
\hline NBS & National Bureau of Standards \\
\hline Newpont News & Newport News Shipbuilding \& Dry Dock Co. \\
\hline NRDS & Nuclear Rocket Development Station \\
\hline NRL & Naval Research Laboratory \\
\hline NSA & Nuclear Systems Associates \\
\hline NTS & Nevada Tert Site \\
\hline NYSC & New Yort Shipbuilding Corp. \\
\hline ORNL & Oak Ridge National Laboratory \\
\hline PNL & Pacific Northwest Laboratory \\
\hline Ponsmouth & Portsmouth Naval Shipyard \\
\hline PPC & Phillips Petroleum Ca. \\
\hline PRDC & Power Reactor Development Company \\
\hline $\mathbf{R I}$ & Rockwell Intemational \\
\hline Sandia & Sandia National Laboratories \\
\hline UNC & United Nuclear Corporation, Development Division \\
\hline Viuro & Vitro Corporation of America \\
\hline West. & Westinghouse Electric Corporation \\
\hline WHC & Westinghouse Hanford Co. \\
\hline
\end{tabular}




\section{REACTORS AND FACILITIES OPERABLE, BEING BUILT, OR PLANNED}


REACTORS AND FACILITIES OPERABLE, BEING BUILT, OR PLANNED

\section{POWER REACTORS}

\section{A. Central-Station Electric Power Plants}

$$
\text { Napee (licensec) [docket number] }
$$

\section{OPERABLE}

Alvin W. Vogtle Nuclear Plmin, Unit 1

(Georgia Power Co.) [50-424]

Alvin W. Vogtle Nuclear Plint, Unit 2

(Georgin Power Co.) [50-425]

Arkanass Nuclear One, Unit 1 (Entergy

Operations Inc.) [50-313]

Atkanats Nuclesr One, Unit 2 (Entergy

Opentioas Inc.) [50-368]

Beaver Valley Power Strtion, Unit 1 (Duqueene

Light Ca, Ohio Edison Ca.) [50-334]

Beaver Valley Power Strtion, Unit 2 (Duquesne

Light $\mathrm{Ca}$ ) [50-412]

Big Rock Point Nuclear Plant (Consumers

Power Ca) [50-155]

Bniducod Sention Unis

(Commonwealth Edivon Co.) [50-456]

Bridwood Sution, Unit 2

(Commonwealth Edieon Co.) [50-457]

Browns Ferry Noclear Power Station, Unit 1

(Tenneasee Valley Authority) [50-259]

Browns Fenry Nuclear Power Station, Unit 2

(Tennessee Valley Authority) [50-260]

Browns Fenry Nuclear Power Sintion, Unit 3

(Tennessee Valley Authority) [50-296]

Brunswick Steam Electric Plene, Unit 1

(Crolina Power \& Light Co.) [50-325]

Brunswick Steam Electric Plant, Unit 2

(Curolina Power \& Light Co.) [50-324]

Byron Straion, Unit 1 (Commonwealth

Edieon Co.) [S0-454]

Byron Strion, Unit 2 (Common wealth

Edivon C0.) [50-455]

Collaway Plant, Unit 1 (Union Electric Co.)

[50-483]

Calven Cliffs Nuclear Power Plane, Unit 1

(Bahimore Gas \& Electric $\mathrm{Co}$ ) [50-317]

Calven Cififs Nuclear Power Phan, Unit 2

(Bahimore Gas \& Electric Co.) [50-318]

Cenubu Noclear Sinion, Unit

(Duke Power Co.) [50-413]

\begin{tabular}{|c|c|c|c|c|c|}
\hline Location & $\begin{array}{l}\text { Princlpel } \\
\text { nuclear } \\
\text { contractor }\end{array}$ & Type & $\begin{array}{c}\text { Power } \\
\text { MD capactly } \\
\text { wet MWT(e) }\end{array}$ & $\begin{array}{l}\text { Power } \\
\text { Llcensed } \\
\text { MW( })\end{array}$ & $\begin{array}{c}\text { Inltial } \\
\text { criticality } \\
\text { (yr mo) }\end{array}$ \\
\hline Waynesboro, GA & Wext. & $\begin{array}{l}\text { Pressurized } \\
\text { water }\end{array}$ & 1158.0 & 3565.0 & 8703 \\
\hline Wayneaboro, GA & West. & $\begin{array}{l}\text { Pressurized } \\
\text { water }\end{array}$ & 1157.0 & 3565.0 & 8903 \\
\hline Russellville, AR & B\&W & $\begin{array}{l}\text { Pressurized } \\
\text { water }\end{array}$ & 836.0 & 2568.0 & 7408 \\
\hline Russellville, AR & Comb. & $\begin{array}{l}\text { Pressurized } \\
\text { water }\end{array}$ & 858.0 & 2815.0 & 7812 \\
\hline Shippineport, PA & Wear. & $\begin{array}{l}\text { Pressurized } \\
\text { water }\end{array}$ & 810.0 & 2652.0 & 7605 \\
\hline Shippingport, PA & Weat. & $\begin{array}{l}\text { Pressurized } \\
\text { water }\end{array}$ & 820.0 & 2652.0 & 8708 \\
\hline Bis Rock Point, MI & GE & Boiling water & 67.0 & 240.0 & 6209 \\
\hline Bnidwood, IL & West. & $\begin{array}{l}\text { Pressurized } \\
\text { water }\end{array}$ & 1120.0 & 3411.0 & 8705 \\
\hline Brnidwood, IL & West. & $\begin{array}{l}\text { Pressurized } \\
\text { water }\end{array}$ & 1120.0 & 3411.0 & 8803 \\
\hline Decatur, AL & $\mathbf{G E}$ & Boiling water & 0.0 & 3293.0 & 7308 \\
\hline Decatur, AL & $\mathbf{G E}$ & Boiling water & 1065.0 & 3293.0 & 7407 \\
\hline Decatur, AL & GE & Boiling water & 0.0 & 3293.0 & 7608 \\
\hline Southport, NC & GE & Boiling water & 767.0 & 2436.0 & 7610 \\
\hline Southpor, NC & $\mathbf{G E}$ & Boiling water & 754.0 & 2436.0 & 7503 \\
\hline Byron, II & West. & $\begin{array}{l}\text { Pressurized } \\
\text { water }\end{array}$ & 1105.0 & 3411.0 & 8502 \\
\hline Byron, IL & Wer. & $\begin{array}{l}\text { Pressurized } \\
\text { yater }\end{array}$ & 1105.0 & 3411.0 & 8701 \\
\hline Fuhon, MO & Wer. & $\begin{array}{l}\text { Pressurized } \\
\text { water }\end{array}$ & 1120.0 & 3565.0 & 8410 \\
\hline Lusby, MD & Comb. & $\begin{array}{l}\text { Pressurized } \\
\text { water }\end{array}$ & 830.0 & 2700.0 & 7410 \\
\hline Lusby, MD & Comb. & $\begin{array}{l}\text { Pressurized } \\
\text { water }\end{array}$ & 830.0 & 2700.0 & 7611 \\
\hline Lake Wylie, SC & Weat. & $\begin{array}{l}\text { Prescurized } \\
\text { water }\end{array}$ & 1129.0 & 3411.0 & 8501 \\
\hline
\end{tabular}

\section{PART I CIVILIAN REACTORS (DOMESTIC)}

Meximum dependable capacity is zero. Administrative hold to resoive various TVA and NRC concems 6/1/85. Restarted 5/23/91.

Maximum dependable capacity is zero. Administrative hold wo resolve various TVA and NRC concems 3/3/85. 


\section{A. Central-Station Electric Power Plants (Continued)}

\begin{tabular}{|c|c|c|c|c|c|c|c|}
\hline Name (licensect) [docket number] & Lacation & $\begin{array}{l}\text { Prindpol } \\
\text { enclear } \\
\text { comtinactor }\end{array}$ & Type & $\begin{array}{l}\text { Ponver } \\
\text { MD capacity } \\
\text { net MW(e) }\end{array}$ & $\begin{array}{l}\text { Power } \\
\text { Leensed } \\
\text { MW(b) }\end{array}$ & $\begin{array}{l}\text { Intial } \\
\text { crifically } \\
(\mathrm{r}=\mathrm{mo})\end{array}$ & Comment \\
\hline $\begin{array}{l}\text { OPERABLE (Continged) } \\
\text { Cornwba Noclear Sinion, Unit } 2\end{array}$ & I te Wylie SC & Weet & & & 3411.0 & 8605 & \\
\hline $\begin{array}{l}\text { (Dule Power Ca.) [50-414] } \\
\text { Chinton Power Station, Unil } 1 \\
\text { (mincis Power Ca.) [50-461] }\end{array}$ & Cineon, IL & Went & $\begin{array}{l}\text { Poiling water } \\
\text { Bolling }\end{array}$ & 930.0 & 2894.0 & 8702 & \\
\hline $\begin{array}{l}\text { Cosanche Peak Stem Electric Station, Unit } 1 \\
\text { (Texas Uvilities Genenting Co.) [50-445] }\end{array}$ & Glen Rose, TX & Wex. & $\begin{array}{l}\text { Pressurized } \\
\text { water }\end{array}$ & 1150.0 & 3411.0 & 9004 & \\
\hline $\begin{array}{l}\text { Comanche Ptak Stemn Electric Station, Unit } 2 \\
\text { (Texas Utilivies Generating Ca.) [50-446] }\end{array}$ & Glen Rose, TX & Wea. & $\begin{array}{l}\text { Pressurized } \\
\text { water }\end{array}$ & 1150.0 & 3411.0 & 9308 & Commencial operntion, 8/3/93. \\
\hline $\begin{array}{l}\text { Cooper Nuclear Station (Nebracta Public } \\
\text { Power Disinia) [50-298] }\end{array}$ & Brownville, NE & GE & Boiling water & 764.0 & 2381.0 & 7402 & \\
\hline $\begin{array}{l}\text { Cyysual River Nuclear Pland, Unit } 3 \\
\text { (Flonida Power Corp.) [50-302] }\end{array}$ & Red Level, FL & B\&W & $\begin{array}{l}\text { Pressurized } \\
\text { water }\end{array}$ & 821.0 & 2544.0 & 7701 & \\
\hline $\begin{array}{l}\text { Davis-Besse Nucless Power Statioa, Unit } 1 \\
\text { (Toledo Edison Co.) [50-346] }\end{array}$ & Oak Harbor, OH & B\&W & $\begin{array}{l}\text { Pressurized } \\
\text { water }\end{array}$ & 877.0 & 27720 & 7708 & \\
\hline $\begin{array}{l}\text { Disblo Cenyon Nuclear Power Plant, Unit } 1 \\
\text { (Pucific Gas \& Electric Ca.) [50-275] }\end{array}$ & Diablo Canyon, CA & Wea. & $\begin{array}{l}\text { Pressurized } \\
\text { water }\end{array}$ & 1073.0 & 3338.0 & 8404 & \\
\hline $\begin{array}{l}\text { Diablo Canyon Nuclear Power Plant, Unit } 2 \\
\text { (Pacific Gas \& Electric Co.) [50-323] }\end{array}$ & Diablo Cenyon, CA & West. & $\begin{array}{l}\text { Pressurized } \\
\text { water }\end{array}$ & 1087.0 & 3411.0 & 8508 & \\
\hline $\begin{array}{l}\text { Donald C. Cook Nuclear Power Plent, Unit } 1 \\
\text { (Indian and Michigan Electric Co.) [S0-315] }\end{array}$ & Bridgman, MI & Wer. & $\begin{array}{l}\text { Pressurized } \\
\text { water }\end{array}$ & 1000.0 & 3250.0 & 7501 & \\
\hline $\begin{array}{l}\text { Domald C. Cook Nuclear Power Plant, Unit } 2 \\
\text { (tndima and Michigen Electric Co.) }[50-316]\end{array}$ & Bridgman, MI & West. & $\begin{array}{l}\text { Pressurized } \\
\text { water }\end{array}$ & 1060.0 & 3411.0 & 7803 & \\
\hline $\begin{array}{l}\text { Dresden Nuclear Power Sution, Unit } 2 \\
\text { (Commonwealth Edison Ca) [SO-237] }\end{array}$ & Morris, IL & GE & Boiling water & 7720 & 2527.0 & 7001 & \\
\hline $\begin{array}{l}\text { Dresden Nuclear Power Surion, Unix } 3 \\
\text { (Commonwealuh Edison Ca) [50-249] }\end{array}$ & Morris, Il & GE & Boiling water & 773.0 & 2527.0 & 7101 & \\
\hline $\begin{array}{l}\text { Duane Amold Energy Cencer, Unit } 1 \text { (lowa } \\
\text { Electric Light \& Power Ca.) [50-331] }\end{array}$ & Palo, IA & $\mathbf{G E}$ & Boiling water & 515.0 & 1658.0 & 7403 & \\
\hline $\begin{array}{l}\text { Edwin I. Hauch Nuclear Plane, Unil } 1 \\
\text { (Georgia Power Ca.) [50-321] }\end{array}$ & Baxley, GA & GE & Boiling water & 737.0 & 2436.0 & 7409 & \\
\hline $\begin{array}{l}\text { Edwin I. Hatch Nuclear Plans, Unit } 2 \\
\text { (Georgia Power Ca) [50-366] }\end{array}$ & Baxley, GA & GE & Boiling water & 757.0 & 2436.0 & 7807 & \\
\hline $\begin{array}{l}\text { Enrico Fermi Alomic Power Plant, Unit } 2 \\
\text { (Detroit Edison Co.) [50-341] }\end{array}$ & Newpor, MI & GE & Boiling water & 1085.0 & 3430.0 & 8506 & \\
\hline $\begin{array}{l}\text { Fon Calhown Sution, Uni } 1 \text { (Omaha } \\
\text { Public Power District) [50-285] }\end{array}$ & Fon Calhown, NE & Comb. & $\begin{array}{l}\text { Pressurized } \\
\text { water }\end{array}$ & 478.0 & 1500.0 & 7308 & \\
\hline $\begin{array}{l}\text { Grand Gulf Nuclear Station, Unit } 1 \\
\text { (Entergy Operations Inc.) [50-416] }\end{array}$ & Pon Gibeon, MS & GE & Boiling water & 1143.0 & 3833.0 & 8208 & \\
\hline $\begin{array}{l}\text { H.B. Robinson Plant, Unit } 2 \text { (Carolina } \\
\text { Power \& Light Co.) [50-261] }\end{array}$ & Hantsville, SC & West. & $\begin{array}{l}\text { Pressurized } \\
\text { water }\end{array}$ & 683.0 & 2300.0 & 7009 & \\
\hline $\begin{array}{l}\text { Haddam Nect Plant (Connecticut Yankee } \\
\text { Alomic Power Co.) [50-213] }\end{array}$ & Haddam Neck, CT & Wext. & $\begin{array}{l}\text { Presserized } \\
\text { water }\end{array}$ & 560.0 & 1825.0 & 6707 & \\
\hline $\begin{array}{l}\text { Hope Creek Nuclear Generating Surtion, Unit } 1 \\
\text { (Public Service Electric \& Gas Ca) [50-354] }\end{array}$ & Salem, NI & $\mathbf{G E}$ & Boiling water & 1031.0 & 3293.0 & 8606 & \\
\hline $\begin{array}{l}\text { Indian Point Station, Unit } 2 \text { (Consolidated } \\
\text { Edison Co. of New Yort, Inc) [50-247] }\end{array}$ & Buchanan, NY & West. & $\begin{array}{l}\text { Pressurized } \\
\text { water }\end{array}$ & 951.0 & 3071.0 & 7305 & \\
\hline
\end{tabular}




\begin{tabular}{|c|c|c|c|c|c|c|}
\hline $\begin{array}{l}\text { Indian Point Siation, Unit } 3 \text { (New York } \\
\text { Power Authority) [50-286] }\end{array}$ & Buchanan, NY & Weat. & $\begin{array}{l}\text { Pressurized } \\
\text { waler }\end{array}$ & 965.0 & 3025.0 & 7604 \\
\hline $\begin{array}{l}\text { James A. FitzPatrick Nuclear Power Plant } \\
\text { (New York Power Authority) [50-333] }\end{array}$ & Scribn, $\mathbf{N Y}$ & $\mathbf{G E}$ & Boiling water & 780.0 & 2436.0 & 7411 \\
\hline $\begin{array}{l}\text { Joseph M. Farley Nuclear Plent, Unit } 1 \\
\text { (Scuthem Nuclear Operating Ca) [50-348] }\end{array}$ & Dothan, AL & Weat. & $\begin{array}{l}\text { Pressurized } \\
\text { water }\end{array}$ & 812.0 & 2652.0 & 7708 \\
\hline $\begin{array}{l}\text { Joscph M. Fartey Nuclear Plant, Unit } 2 \\
\text { (Southem Nuclear Operating Co.) [50-364] }\end{array}$ & Dothan, AL & West. & $\begin{array}{l}\text { Pressurized } \\
\text { water }\end{array}$ & 822.0 & 2652.0 & 8105 \\
\hline $\begin{array}{l}\text { Kewaunce Nuclear Power Plant (Wisconsin } \\
\text { Public Service Corp.) [50-305] }\end{array}$ & Cartion, WI & West. & $\begin{array}{l}\text { Pressurized } \\
\text { water }\end{array}$ & 511.0 & 1650.0 & 7403 \\
\hline $\begin{array}{l}\text { La Salle County Station, Unit } 1 \\
\text { (Commonwealth Edison Co.) [50-373] }\end{array}$ & Seneca, II & GE & Boiling water & 1036.0 & 3323.0 & 8206 \\
\hline $\begin{array}{l}\text { La Salle County Station, Unit } 2 \\
\text { (Commonwealth Edison Ca.)[50-374] }\end{array}$ & Seneca, II & $\mathbf{G E}$ & Boiling weter & 1036.0 & 3323.0 & 8403 \\
\hline $\begin{array}{l}\text { Limerick Generating Station, Unit } 1 \\
\text { (Philadelphia Electric Co.) [50-352] }\end{array}$ & Potstown, PA & GE & Boiling water & 1055.0 & 3293.0 & 8412 \\
\hline $\begin{array}{l}\text { Limerick Generating Station, Unit } 2 \\
\text { (Philadelphia Electric Co.) [50-353] }\end{array}$ & Poustown, PA & GE & Boiling water & 1055.0 & 3293.0 & 8908 \\
\hline $\begin{array}{l}\text { Maine Yenkee Atomic Power Plant (Maine } \\
\text { Yankee Alomic Power Co.) [50-309] }\end{array}$ & Wiscassec, ME & Comb. & $\begin{array}{l}\text { Pressurized } \\
\text { water }\end{array}$ & 860.0 & 2700.0 & 7210 \\
\hline $\begin{array}{l}\text { Millstone Nuclear Power Station, Unit 1 } \\
\text { (Nontheast Nuclear Energy Ca.) [50-245] }\end{array}$ & Waterford, CT & GE & Boiling water & 641.0 & 2011.0 & 7010 \\
\hline $\begin{array}{l}\text { Millstone Nuclear Power Station, Unit } 2 \\
\text { (Nontheast Nuclear Energy Ca.) [50-336] }\end{array}$ & Waterford, CT & Comb. & $\begin{array}{l}\text { Pressurized } \\
\text { water }\end{array}$ & 873.0 & 2700.0 & 7510 \\
\hline $\begin{array}{l}\text { Millstone Nuclear Power Station, Unit } 3 \\
\text { (Northeast Nuclear Energy Ca.) [50-423] }\end{array}$ & Waterford, CT & West. & $\begin{array}{l}\text { Pressurized } \\
\text { water }\end{array}$ & 1137.0 & 3411.0 & 8601 \\
\hline $\begin{array}{l}\text { Monticello Nuclear Generating Plant } \\
\text { (Northem States Power Co.) [50-263] }\end{array}$ & Monticello, MN & GE & Boiling water & 536.0 & 1670.0 & 7012 \\
\hline $\begin{array}{l}\text { Nine Mile Point Nuclear Station, Unit } 1 \\
\text { (Ningar Mohawk Power Corp.) [50-220] }\end{array}$ & Scribe, NY & GE & Boiling water & 565.0 & 1850.0 & 6909 \\
\hline $\begin{array}{l}\text { Nine Mile Point Nuclear Station, Unit } 2 \\
\text { (Niagare Mohawk Power Corp.) }[50-410]\end{array}$ & Scriba, NY & GE & Boiling water & 994.0 & 3323.0 & 8705 \\
\hline $\begin{array}{l}\text { Nonh Anna Power Station, Unil } 1 \\
\text { (Virginia Electric \& Power Ca) } \\
\text { [50-338] }\end{array}$ & Mineral, VA & West. & $\begin{array}{l}\text { Pressurized } \\
\text { water }\end{array}$ & 900.0 & 2893.0 & 7804 \\
\hline $\begin{array}{l}\text { North Anma Power Station, Unit } 2 \\
\text { (Virginia Electric \& Power Co.) [50-339] }\end{array}$ & Mineral, VA & West. & $\begin{array}{l}\text { Pressurized } \\
\text { water. }\end{array}$ & 887.0 & 2893.0 & 8006 \\
\hline $\begin{array}{l}\text { Oconee Nuclear Sution, Unit } 1 \text { (Duke Power } \\
\text { Co.) [50-269] }\end{array}$ & Seneca, SC & B\&W & $\begin{array}{l}\text { Pressurized } \\
\text { water. }\end{array}$ & 846.0 & 2568.0 & 7304 \\
\hline $\begin{array}{l}\text { Oconcee Nuclear Station, Unit } 2 \text { (Duke Power } \\
\text { Co.) [50-270] }\end{array}$ & Seneca, SC & B\&W & $\begin{array}{l}\text { Pressurized } \\
\text { water }\end{array}$ & 846.0 & 2568.0 & 7311 \\
\hline $\begin{array}{l}\text { Oconec Nuclear Station, Unit } 3 \text { (Duke Power } \\
\text { Co.) [50-287] }\end{array}$ & Seneca, SC & B\&W & $\begin{array}{l}\text { Pressurized } \\
\text { water }\end{array}$ & 846.0 & 2568.0 & 7409 \\
\hline $\begin{array}{l}\text { Oyster Croek Nuclear Power Piant, Unit } 1 \\
\text { (GPU Nuclear Corp.) [50-219] }\end{array}$ & Toms River, NJ & GE & Boiling water & 610.0 & 1930.0 & 6905 \\
\hline $\begin{array}{l}\text { Palisades Nuclear Plant, Unit } 1 \\
\text { (Consumers Power Co.) [50-255] }\end{array}$ & South Haven, MI & Comb. & $\begin{array}{l}\text { Pressurized } \\
\text { water }\end{array}$ & 730.0 & 2530.0 & 7105 \\
\hline $\begin{array}{l}\text { Palo Verde Nuclear Generating Station, Unit } 1 \\
\text { (Arizona Public Service Co.) [50-528] }\end{array}$ & Wintersburg, AZ & Comb. & $\begin{array}{l}\text { Pressurized } \\
\text { water }\end{array}$ & 1221.0 & 3800.0 & 8505 \\
\hline $\begin{array}{l}\text { Palo Verde Nuclear Generaing Station, Unit } 2 \\
\text { (Arizona Public Service Co.) [50-529] }\end{array}$ & Wintersburg, AZ & Comb. & $\begin{array}{l}\text { Pressurized } \\
\text { water }\end{array}$ & 1221.0 & 3800.0 & 8604 \\
\hline $\begin{array}{l}\text { Palo Verde Nuclear Generating Station, Unit } 3 \\
\text { (Arizona Public Service Co.) [50-530] }\end{array}$ & Wintersburg, AZ & Comb. & $\begin{array}{l}\text { Pressurized } \\
\text { water }\end{array}$ & 1304.0 & 3817.0 & 8710 \\
\hline $\begin{array}{l}\text { Peach Bottom Atomic Power Station, Unit } 2 \\
\text { (Philadelphia Electric Co.) [50-277] }\end{array}$ & Lencaster, PA & $\mathbf{G E}$ & Boiling water & 1055.0 & 3293.0 & 7309 \\
\hline $\begin{array}{l}\text { Peach Bottom Atomic Power Station, Unit } 3 \\
\text { (Philadelphia Electric Co.) [50-278] }\end{array}$ & Lancaster, PA & GE & Boiling water & 1035.0 & 3293.0 & 408 \\
\hline
\end{tabular}




\section{A. Central-Station Electric Power Plants (Continued)}

\section{Name (licensec) [dockế number]}

OPERABLE (Contimued)

Pery Nuclear Power Plant, Unit 1 (Cleveland Eloctric Illuminating $C_{0}$.) $(50-440)$

Pilgrim Nuclear Power Stution, Unit 1

(Bocton Edison Co.) [50-293]

Point Beach Nuclear Plane, Unit

(Wisconsin Electric Power Co.) [50-266]

Point Beach Nuclear Plant, Unit 2

(Wisconsin Electric Power Co.) [50-301]

Privie Island Nuclear Generating Plant, Unit 1

(Northem Sintes Power Co.) [50-282]

Privie Island Nuclear Generating Plant, Unit 2

(Northem Sintes Power Co.) [50-306]

Oand-Citiea Siation, Unit 1 (Commonwealh

Edison C0.) [50-254]

Quad-Cities Station, Unit 2 (Commonwealth

Edison Co.) [50-265]

- River Bend Station, Unit 1 (Gulf States

Unilities Co.) [50-458]

Roben Emmen Ginna Nuclear Power Plant,

Unit 1 (Rochester Gas \& Electric Corp.)

[50-244]

Salem Nuclear Generating Station, Unit 1

(Public Service Electric \& Gas Ca) [50-272]

Salem Nuclear Generating Station, Unit 2

(Public Service Electric \& Gas Co.) [50-311]

(Southem California Edison) [50-361]

San Onofre Nuclear Generating Station, Unit 3

(Southem California Edison) [50-362]

Seabrook Nuclear Station, Unit 1 (North

Adentic Energy Service Corp.) [50-443]

Sequoyah Nuclear Plant, Unit 1

(Tennessee Valley Authority) [50-327]

Sequoyah Nuclear Plant, Unit 2

(Tennessee Valley Authority) [50-328]

Shearon Harris Nuclear Power Plant, Unit

(Carolina Power \& Light Co.) [50-400]

South Texas Project, Unit 1 (Houston

Lighing \& Power Co.) [50-498]

Sowh Texas Project, Unit 2 (Houston

Lighting \& Power Co.) [50-499]

SL Lucie Plant, Unit 1 (Florids Power \&

Light $\mathrm{Ca}$ ) [50-335]

SL. Lucie Plant, Unir 2 (Florida Power \&

Light $\mathrm{Ca}$ ) [50-389]

$\begin{gathered}\text { Principad } \\ \text { nuclear } \\ \text { contractor }\end{gathered}$
Location

Perry, OH

Plymouth, MA

GE

Two Creeks, WI

GE

Two Creeks, WI

Red Wing, MN

Red Wing, MN

Cordova, IL

Cordova, IL

St. Frnncisville, LA

Ontario, NY

Salem, NJ

Salem, NJ

San Clemente, CA

San Clemente, CA

Seabrook, NH

Daisy. TN

Daisy, TN

Bonsal, NC

Bay City, TX

Bay City, TX

Fon Pierce, FL.

Fon Pierce, FI

West.

West.

West.

West.

GE

GE

GE

Comb.

West.

West.

Type

Power

Power

Licensed crilicality

net $M W(e) \quad M W(t) \quad$ (jr mo)

Boiling water

$1166.0 \quad 3579.0 \quad 8606$

Boiling water

670.0

$1998.0 \quad 7206$

Pressurized

water

Pressunized

water

Pressurized

water

Pressurized

water

Boiling water

Boiling water

485.0

$1519.0 \quad 7011$

485.0

$1519.0 \quad 7205$

513.0

$1650.0 \quad 7312$

$512.0 \quad 1650.0 \quad 7412$

$\begin{array}{lll}769.0 & 2511.0 \quad 7110\end{array}$

769.0

$2511.0 \quad 7204$

Boiling water

936.0

$2894.0 \quad 8510$

West Pressurized

water

West. Pressurized

470.0

$1520.0 \quad 6911$

$1106.0 \quad 3411.0 \quad 7612$

West. Pressurized

Pressurized

water

Pressurized

water

Pressurized

water

West. Pressurized

West. Pressurized

West. Pressurized

West. Pressurized

Pressurized

water

Pressurized

water

Pressurized

water 
Surn Power Sation, Unit 1 (Virginia Blectric a Power Ca) [SO-280] Surry Power Stetion, Uhit 2 (Vipjinin Electric \& Power Ca) [S0-281]

Suequeheras Stom Floctic Strion, Unit 1 (Pennoyivanin Power \& Light Co.) [50-387] Suequeharan Stonan Bloctric Simion, Unit 2 (Pennoytvanio Power \& Ligh Ca) [50-388] Three Mile Isluad Noclear Stration, Unit 1 (GPU Nuclear Corp) (50-289]

Tutkey Point Phane, Unil 3 (Florid

Power \& Lighs $\mathrm{Ca}$ ) (50-250]

Turkey Point Plene, Unit 4 (Florid

Power \& Lighs $\mathrm{Ca}$ ) [50-251]

Vermona Yaskee Nuclear Power Station

(Vermoan Yankee Nuclear Power Corp.)

[50-271]

Virgil C. Summer Nuclear Strtion, Unit 1 (South Carolim Electric \& Gas Co.) [50-395]

Washington Nuclear Project, Unit 2 (Wathing-

con Public Power Supply Syuem) [50-397]

Wreefond Genenting Sintion, Unit 3

(Enterny Opercions Inc.) [50-382]

Willien B. MeCuire Nuclear Sution

Unit 1 (Duke Power CO.) [S0-369]

Willim B. Mccuire Nuclear Station,

Unis 2 (Duke Power Ca) [50-370]

Wolf Creek Genemtin Sintion

(Wolf Creek Nuclear Opernting Corp.)

[50-482]

Zion Nectear Phom Unit 1 (Comsmonwealhh

Edison Co.) [50-295]

Zion Nuclear Phen, Unit 2 (Commonwealth

Edison Ca.) [50-304]

\begin{tabular}{|c|c|c|c|c|c|}
\hline Grovel Nect, VA & Wear. & Pressurized & 781.0 & 2441.0 & 7207 \\
\hline Gravel Neck, VA & Wea. & $\begin{array}{l}\text { Prosencized } \\
\text { wher }\end{array}$ & 781.0 & 2441.0 & 7303 \\
\hline Berwick, PA & $\mathbf{G E}$ & Boiline water & 1040.0 & 3293.0 & 8209 \\
\hline Benwick, PA & GE & Boiling water & 1044.0 & 3293.0 & 8405 \\
\hline Midallecown, PA & BeW & Pressurized & 786.0 & 2568.0 & 7406 \\
\hline Floride Ciry, FL & Weat. & $\begin{array}{l}\text { Prossurized } \\
\text { water }\end{array}$ & 666.0 & 2200.0 & 7210 \\
\hline Florida City, FL & Weat. & $\begin{array}{l}\text { Pressurized } \\
\text { water }\end{array}$ & 666.0 & 2200.0 & 7306 \\
\hline Vemon, VT & GE & Boiling waser & 504.0 & 1593.0 & 7203 \\
\hline Jenkinsville, SC & Weat. & $\begin{array}{l}\text { Pressurized } \\
\text { water }\end{array}$ & 885.0 & 2775.0 & 8210 \\
\hline Richland, WA & GE & Boiling water & 1086.0 & 3323.0 & 8401 \\
\hline Taft, LA & Comb. & $\begin{array}{l}\text { Presenurized } \\
\text { water }\end{array}$ & 1075.0 & 3390.0 & 8503 \\
\hline $\begin{array}{l}\text { Cowens Fond Dem, } \\
\text { NC }\end{array}$ & Wear. & $\begin{array}{l}\text { Pressurized } \\
\text { wreter }\end{array}$ & 1129.0 & 3411.0 & 8103 \\
\hline $\begin{array}{l}\text { Cowrans Ford Dam, } \\
\text { NC }\end{array}$ & Weat. & $\begin{array}{l}\text { Pressurized } \\
\text { waller }\end{array}$ & 1129.0 & 3411.0 & 8305 \\
\hline Burtingtion, KS & Weat. & Pressurized & 1134.0 & 3565.0 & 8505 \\
\hline Zion, Il & Weat. & $\begin{array}{l}\text { Preasurized } \\
\text { water }\end{array}$ & 1040.0 & 3250.0 & 7306 \\
\hline Zion, II & Weat. & $\begin{array}{l}\text { Pressurized } \\
\text { water }\end{array}$ & 1040.0 & 3250.0 & 7312 \\
\hline Loention & $\begin{array}{l}\text { Princlpal } \\
\text { nuclear } \\
\text { contractor }\end{array}$ & Type & $\begin{array}{l}\text { Desion, } \\
\text { electerical } \\
\text { power } \\
\text { aet MW(e) }\end{array}$ & $\begin{array}{l}\text { Dexish, } \\
\text { thermal } \\
\text { powes } \\
\text { ie MW(t) }\end{array}$ & $\begin{array}{l}\text { Sedimated } \\
\text { Imidial } \\
\text { criticality } \\
\text { (jr moo) }\end{array}$ \\
\hline
\end{tabular}

Name (licensec) [docket number]

Scousboro, AL

BaW

Pressarized

Bellefonte Nuclear Plan, Unit 1

(Tennessee Valley Ambority) [50-438]

Bellefonte Nuclear Plane, Unir 2

(Tennessee Valley Ambority) [50-439]

Perny Nuclear Power Plane, Unit 2 (Cleveland

Electric Muminating $\mathrm{Co}$.) [50-441]

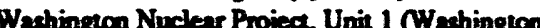

Public Power Supply System) [50-460]

Washingten Nuclear Project, Unit 3 (Weshington

Public Power Supply System) [50-508]

Watts Bar Nuclear Plang, Unit 1

(Tennessee Valley Auchority) [50-390]

Wans Bar Nuclear Plan, Unit 2

(Temessee Valley Ambority) [50-391]
Soousboro, AL BEW Pressurized

Perry, OH

Richland, WA

Sotsop, WA

Spring Ciry, TN

Spring City, TN
GE

water

Boiling water

1235.0

1205.0

1266.0

1242.0

water

Comb.

Wear.

Pressurized

Presertrized

water

West.

water
Pressurized
vater

$1165.0 \quad 3411.0 \quad$ Indef
Comment

3760.0 Indef.

3759.0 Indef.

3760.0 indef.

3800.0 Indef 
B. Dualpurpose Plants

(No reactors currently in this category)

C. Propulsion (Maritime)

(No reactors currently in this calegory)

\section{EXPERIMENTAL POWER-REACTOR SYSTEMS}

\section{A. Electric-Power Systems}

a

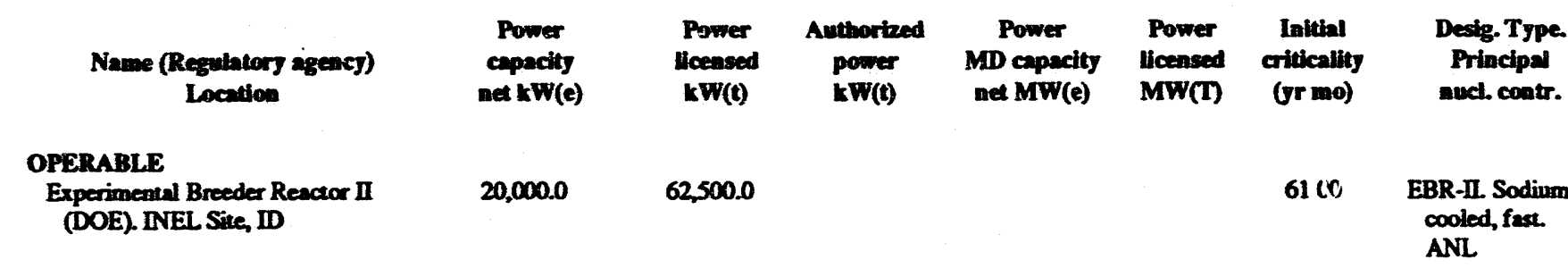

B. Space Nuclear Auxillary Power (SNAP)

(No reactors currently in this category)

C. Space Propulsion

(No reactors currently in this category) 


\section{TEST, RESEARCH, AND UNIVERSITY REACTORS}

\section{A. General Irradiation Test}

\begin{tabular}{|c|c|c|c|c|c|c|c|c|}
\hline $\begin{array}{l}\text { Name (Reguhtory agency). } \\
\text { Location }\end{array}$ & $\begin{array}{c}\text { Power } \\
\text { cappacty } \\
\text { net } \mathbf{k W}(e)\end{array}$ & $\begin{array}{c}\text { Power } \\
\text { Hicensed } \\
k W(t)\end{array}$ & $\begin{array}{l}\text { Authorized } \\
\text { power } \\
k W(t)\end{array}$ & $\begin{array}{l}\text { Power } \\
\text { MD capactty } \\
\text { net } M W(e)\end{array}$ & $\begin{array}{l}\text { Power } \\
\text { licensed } \\
\text { MW(I) }\end{array}$ & $\begin{array}{l}\text { Inithal } \\
\text { crilicality } \\
\text { (jr mo) }\end{array}$ & $\begin{array}{l}\text { Desig. Type. } \\
\text { Principal } \\
\text { wucl. contr. }\end{array}$ & Comment \\
\hline \multicolumn{9}{|l|}{ OPERABLE } \\
\hline $\begin{array}{l}\text { Advanced Test Reactor (DOE). } \\
\text { INEL, ID }\end{array}$ & & & $250,000.0$ & & & 6800 & $\begin{array}{l}\text { ATR. Tank. } \\
\text { EG\&G-ID }\end{array}$ & Operaing. \\
\hline \multirow{2}{*}{\multicolumn{9}{|c|}{$\begin{array}{l}\text { PLANNED } \\
\text { Los Alamos National Lebontory (DOE). } \\
\text { Los Alamos, NM }\end{array}$}} \\
\hline & & & & & & & & \\
\hline \multicolumn{9}{|c|}{ B. High-Power Research and Test } \\
\hline \multicolumn{9}{|l|}{ OPERABLE } \\
\hline $\begin{array}{l}\text { Brookthaven High Flux Beam Research } \\
\text { Reactor (DOE). Upton, NY }\end{array}$ & & & $35,400.0$ & & & 6500 & $\begin{array}{l}\text { HFBR. Heavy } \\
\text { waver. BNL }\end{array}$ & Power dented subject wo further safety lests. \\
\hline $\begin{array}{l}\text { Brookthaven Medical Research Reacior } \\
\text { (DOE). Uplon, NY }\end{array}$ & & & $3,000.0$ & & & 5900 & $\begin{array}{l}\text { BMRR. Tank } \\
\text { Daystrom }\end{array}$ & \\
\hline $\begin{array}{l}\text { High Fux Isocope Reactor (DOE). } \\
\text { Out Ridge, TN }\end{array}$ & & & $85,000.0$ & & & 6500 & $\begin{array}{l}\text { HFIR. Tank flux } \\
\text { trap. ORNL }\end{array}$ & Operating. \\
\hline $\begin{array}{l}\text { National Institure of Sumdands \& } \\
\text { Technology (NRC). Gaithersburg, MD }\end{array}$ & & & $20,000.0$ & & & 6700 & $\begin{array}{l}\text { NIST. Heavy } \\
\text { water. } \\
\text { NBS-B\&R }\end{array}$ & \\
\hline $\begin{array}{l}\text { Omega West Reactor (DOE). Los } \\
\text { Alamos, NM }\end{array}$ & & & $8,000.0$ & & & 5600 & $\begin{array}{l}\text { OWR. Tant: } \\
\text { LANL }\end{array}$ & Potential candidate for a U.S. Mo-9 \\
\hline
\end{tabular}

\section{Safety Research and Test}

\section{OPERABLE}




\section{General Research}

\section{Name (Rezulatory azency). Locetion}

\section{OPERABLE}

Advanced Reactivity Measurement

Facility (DOE). INEL Site, ID

Aerotest Operations, Inc. (NRC).

Sm Remon, CA

Compled Fast Reactivity Measurement

Facility (DOE). INEI. Site, ID

Dow Chemical Co. (NRC). Midlend, MI

Genernl Alomics, Advanced TRIGA-Mk F

Prototype Reactor (NRC). La Jolla, CA

- Genenal Atomica, TRIGA-Mk I

Prototype Reacior (NRC). La Jolla, CA

Geseral Electric Nuclear Test Reactor

(NRC). Pleasaneon, CA
Neutron Radiography Facility (DOE).

INEL, ID

Omaha Veterans Administration
Hospital (NRC). Omaha, NE

Rhode Island Nuclear Science Center

(NRC). Narrengenseth, RI

Sindia Pulsed Reactor II (DOE).

Kirtland AFB, East, NM

\section{Sandia Pulsed Reactor III (DOE)}

Kirland AFB, Eass, NM

\begin{tabular}{|c|c|c|}
\hline \multirow[t]{5}{*}{$\begin{array}{c}\text { Power } \\
\text { capacity } \\
\text { net } L W(e)\end{array}$} & $\begin{array}{c}\text { Power } \\
\text { Hoceased } \\
\text { kW(t) }\end{array}$ & $\begin{array}{l}\text { Authertzed } \\
\text { power } \\
k W(t)\end{array}$ \\
\hline & & 100.0 \\
\hline & & 250.0 \\
\hline & & 100.0 \\
\hline & & 300.0 \\
\hline \multirow[t]{8}{*}{$F$} & & $1,500.0$ \\
\hline & & 250.0 \\
\hline & & 100.0 \\
\hline & & 250.0 \\
\hline & & 18.0 \\
\hline & & $2,000.0$ \\
\hline & & 25.0 \\
\hline & & 25.0 \\
\hline \multirow[t]{2}{*}{ OE). } & & $2,000.0$ \\
\hline & & $1,000.0$ \\
\hline
\end{tabular}

Kinland AFB, East, NM

U.S. Geological Survey Laboratory

(Department of the Interior) (NRC)

Denver, CO

\section{PLANNED}

Advanced Neutron: Source Reactor

(DOE). Oak Ridge, TN

$330,000.0$

$\begin{array}{cccc}\text { Power } & \text { Power } & \text { Initial } & \text { Desig. Type. } \\ \text { critlcallty } & \text { Principal } \\ \text { ned MW(e) } & \text { MWW(T) } & \text { (jr mo) } & \text { nucl. contr. }\end{array}$

Comment

$\begin{array}{cc}6000 & \text { ARMF. Pool. } \\ 6500 & \text { EG\&G-ID } \\ \text { AGNIR. } \\ \text { Pool-TRIGA } \\ \text { core. GA } \\ 6800 \quad \begin{array}{c}\text { CFRMF. Pool. } \\ \text { EG\&G-ID }\end{array}\end{array}$

Fast and thermal neutron irndietions and reactivity measurements. Reacior currently shue down.

Fast and thermal neutron imadiations, neutron ndiography, and thermal and fast fissite

6700 TRIGA-MkI.

U-Zr hydride GA

TRIGA-Mk F.

U-Zr hydride.

Owner

5800 TRIGA-Mk I.

U-ZR hydride

5700 NTR. LWR.

$7700 \quad$ NRAD

Pool-TRIGA

5900 core. ANL

U-Zr hydride.

GA

6400 RINSC.

6700 Pool. RI

SPR-II. Bare

metal fast

burst. Sandia
SPR-III. Bare

metal fast

burst. Sandia

7800 ACRR. Pool-UO

BeO core. Sandia

6900 TRIGA-Mk I.

U-Zr hydride.

GA

Pulse, steady state.

Pulse, steady state.

Pulse, computer transient steady state.
ANS. $\mathrm{D}_{2} \mathrm{O}$ flux trap. ORNL
Advanced conceptual design. In preparation for FY 1995 line item start. 


\section{E. Univeralty Resoarch and Teeching}

\begin{tabular}{|c|c|c|c|c|c|c|}
\hline arequt & $\begin{array}{c}\text { Power } \\
\text { capecily } \\
\text { net k } W(\theta)\end{array}$ & $\begin{array}{l}\text { Power } \\
\text { leaned } \\
\text { kW(T) }\end{array}$ & $\begin{array}{l}\text { Autwertaed } \\
\text { powar } \\
\text { LW(1) }\end{array}$ & $\begin{array}{c}\text { Power } \\
\text { MD capactisy } \\
\text { med MW(t) }\end{array}$ & $\begin{array}{l}\text { Power } \\
\text { llowend } \\
\text { MW(T) }\end{array}$ & $\begin{array}{c}\text { Inledal } \\
\text { critically } \\
\text { (rr mo) }\end{array}$ \\
\hline
\end{tabular}

\section{Oproanes}

Arivons, University of (NRC).

Trescen, A2

Irvine, CA

Comell Univerniry (NRC) Hhace, NY

Comell Universiny Zero Power Reactor (NRC), Inhaca, NY

Florich, Univerrity of (NRC)

Gineoville, FL

Georgin Intimbe of Technology (NRC) Athata, GA

Idaho Sure University (NRC).

Poestella, ID

Itimcis, University of (NRC). Ubeme, II

Minois, Universiry of (NRC)

Chempnize-Uitens, II

Iown Stme University (NRC). Ames, IA

Kmeas Sinde Univenity (NRC)

Manhaman, KS

Menhanen College (NRC)

New Yot, NY

Marylend, University of (NRC)

College Paiversity

\section{Masenchusets, University of (NRC)}

Lowell, MA

Masachuretus Instime of Technolosy

(NRC) Cmabridze, MA

Michisan, Univerrity of (Ford Nuclear

Reaceor) (NRC). Am Arbor, MI

Miseconi a Rolla, Univering of

(NRC). Roll, $\mathrm{MO}$

Miscouri, Universiny of (NRC)

Columbia, MO
100.0

250.0

5800 TRIGA-MkI

U-Zr hydride.

$$
\text { GA }
$$

6900 TRIGA-Mk L

U-Zr hydride.$$
\text { GA }
$$

6200 TRIGA-MK II.

U-Zrhydride.

500.0

100.0

$5,000.0$

6200 ZPR. Tank

Vitro

5900 UFTR.

Grophite/water.

GNEC

6400 GTRR. Heavy

6700 AGN-201P-103

Homos. solid.

$$
\text { AGN }
$$

$1,500.0$

10.0

250.0

250.0

$1,000.0$

$5,000.0$

$2,000.0$

200.0

$10,000.0$
7100 LOPRA. U-Z

hydride. GA

6000 TRIGA-MII

U-Zr hydride.

GA

$5900 \quad$ UTR-10.

Graphize/water.

AS Inc.

6200 TRIGA-Mk II

U-Zs hydride.

GA

6400 MCZPR. Tank.

AMF

7400 TRIGA

Tank-TRIGA

core. GA

7400 ULR. Pool. GE

5800 MITR-II

Heavy-water

reflected $A C$

5700 FNR. Pool. B\&W

6100 UMR-R. Pool. CW

6600

MURR. Tank
Authorized power is segligible.

The AGN-201P-103 was operated at San Ramon,

CA, by Aerojet-General Corporation from 1957

to 1966. In $4 / 67$ Idaho State University applied

for a license to opernite the reactor at Pocatello,

ID. Authorized power is negligible.

Anthorized power is negligible.

Owner-IC 


\section{E. University Rosearch and Teaching (Continued)}

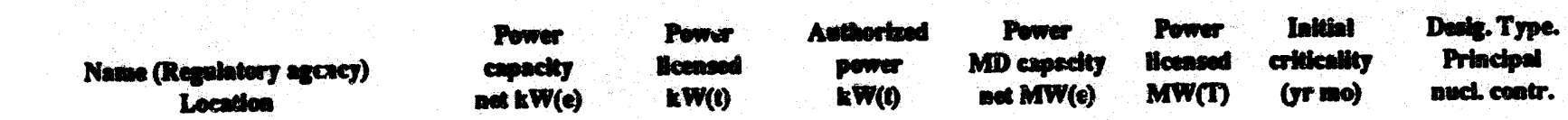

Comment

\section{OPBRABLE (Coadimed)}

Now Mexico, Univerity of (NRC)

Alboquerque, NM

North Carolins Stue Univerxity

(NRC) Raleigh, NC

Otio Stute Univenity (NRC)

Columber, OH

Oregon Stuse University (NRC)

Corvelis, OR

Peran Sute TRIGA Reactor (NRC).

Univernity Paik, PA

Pundue University (NRC).

Wen Lafayetre, IN

Reed College (NRC). Portand, OR

Stute University of New Youk

(NRC), Buffalo, NY

Texas AdM Univerrity (NRC)

College Stution, TX

Texas A\&M University (NRC).

College Sution, TX

Texas at Austin, University of (NRC).

Austin. TX

Utah, Univervity of (NRC). Salt Lake Ciry. UT

Virginia, University of (NRC).

Chardonesville, VA
6600

1,0000

$1,000.0$ AGN-201M-112.
Homes. Solid. ACN

500.0

$1,000.0$

$1,000.0$

1.0

250.0

$2,000.0$

$1,100.0$

250.0

$2,000.0$

8900

TRIGA-Mk II. U-Zr hydride. GA

7500 TRIGA-MkI.

U-Z hydride.

GA

6000 UVAR. Pool.
200 PULSTAR. Pool AMF

6100 OSURR. Pool

Lockbeed

6700 OSTR. U-Zr

hydride. GA

6500 PSTR

Pool-TRIGA core GA

6200 PUR-1. Pool

Lockheed

TRIGA-Mk I.

U-Zr hydride.

GA

6100 PULSTAR P. AMF

5700 AGN-201M-106. Homog. solid AGN

6100 TRIGA. U-Z hydride. GA

Owner: Pennsylvenia State University. From 1955 to 1965, the Pern Sune reactor was operated as a 200kW( $(t)$ poot-type reactor fueled with MTR-type elements.

Owner: Buffalo Maserials Research Center.

Authorized power is negligible.

The Nuclear Science Center Reactor at Texas AdM University has been modified for $1000-\mathrm{kW}$ steadystate operation with a TRIGA-type core. Power level was $100 \mathrm{~kW}$ prior to modification in 1968.
AGN-201M-112 was operuled at the University of Califomia, Betkeley, beginning in 1957. The in 4 es for of at a sive on its campus. The reactor achieved criticality at the University of New Mexico on 107/66. Authorized power is negligible.

Owner-BeW 
Washiagton State University (NRC).

$1,000.0$

Pullman, WA

Wisconsin, University of (NRC)

Madison, WI

Worcester Polyrechnic Institule (NRC).

Worcester, MA

10.0

\section{PLANNED}

Arkansas Tech University (NRC)

Russellville, AR

$1,000.0$

250.0

1. MATERIALS PRODUCTION

\section{PART II PRODUCTION REACTORS}

6700

In 1957 the oritinal MTR-type cose of the

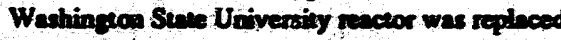

by a modified TRCA type cone sod control yatem, and the steady-state power level was increased fiom 100 20 $1000 \mathrm{~kW}(0)$.

6700 TRIGA The University of Wisconain reactor has been modified

Pool-TRIGA for 1000 $k$ W steady-stace opertion with a TRIGA-

core GA

type core. Power level was $250 \mathrm{~kW}$ prior to

modification in 1967.

TRIGA-Mk I. The Artanas Tech University reactor contuins U-Zr hydride. parts from the TRIGA reactor dismantled at

GA Michigan State University.

Name

Locadion

Nuclear Type

Authorized power

Startup

Comment

\section{OPERABLE}

KReactor (DOE)

Aiken, SC

Du Pont

Heavy water

5400

Cold standby.

\section{PROCESS DEVELOPMENT}




\section{A. Remote installations}

(No reactors currently in this category)

\section{B. Propulsion (Naval)}

The abbreviations used here are defined as follows:

SSN, Submarine (Nuclear Propulsion)

SSBN, Feet Ballistic Mirsile Submarine (Nuclear Propulsion)

CGN, Guided Missile Cruiser (Nuclear Propulsion)

CVN, Airenfi Carrier (Nuclear Propulsion)

Name (all owned by U.S. Navy)

Dexignation

Shipbullder

Startup

USS GATO

DANIEL WEBSTER

USS STONEWALL JACKSON

SAMRAYBURN

USS STURGEON

USS WHALE

USS TAUTOC

N USS SIMON BOLIVAR

USS KAMEHAMEHA

USS JAMES K. POLK

USS GRAYLNG

USS POGY

USS ASPRO

USS SUNFISH

USS PARGO

USS PUFFER

USS MARIANO G. VALLEJO

USS SAND LANCE

USS GURNARD

USS HAMMERHEAD

USS HAWKBILL

USS BERGALL

USS SPADEFISH

USS SEA HORSE

USS FINBACK

USS NARWHAL

USS PINTADO

USS FLYING FISH

USS TREPANG

USS BLUEFISH

USS BILLFTSH

USS DRUM

USS ARCHERFISH

USS SILVERSIDES

USS WILLIAM H. BATES

USS BATFISH
GD (Quincy) $\quad 6700$

\begin{tabular}{|c|c|c|}
\hline $\begin{array}{l}\text { SSN615 } \\
\text { ex-SSBN626 } \\
\text { SSBN634 } \\
\text { ex-SSBN635 } \\
\text { SSN637 }\end{array}$ & $\begin{array}{l}\text { GD (Quincy) } \\
\text { Eloctric Boat (Groton) } \\
\text { Mare Island } \\
\text { Newpon News } \\
\text { Electric Bont (Groton) }\end{array}$ & $\begin{array}{l}6700 \\
6400 \\
6400 \\
6400 \\
6600\end{array}$ \\
\hline SSN638 & GD (Quincy) & 6800 \\
\hline SSN639 & Ingalls & 6800 \\
\hline SSBN641 & Newport News & 6500 \\
\hline SSN642 & Mare Island & 6500 \\
\hline SSN645 & Electric Bout (Grocon) & 6600 \\
\hline SSN646 & Ponsmouth & 6900 \\
\hline SSN647 & NYSC/Ingalls & 7000 \\
\hline SSN648 & Ingalls & 6800 \\
\hline SSN649 & GD (Quincy) & 6800 \\
\hline SSN650 & Electric Boat (Groton) & 6700 \\
\hline SSN652 & Ingalls & 6900 \\
\hline SSBN658 & Mare Island & 6600 \\
\hline SSN660 & Ponsmouth & 7100 \\
\hline SSN662 & Mare Island & 6800 \\
\hline SSN663 & Newpon News & 6700 \\
\hline SSN666 & Mare Island & 7000 \\
\hline SSN667 & Electric Boat (Groton) & 6900 \\
\hline SSN668 & Newpon News & 6900 \\
\hline SSN669 & Electric Boat (Groton) & 6900 \\
\hline SSN670 & Newpon News & 6900 \\
\hline SSN671 & Electric Boat (Groton) & 6900 \\
\hline SSN672 & Mare Island & 7000 \\
\hline SSN673 & Electric Boat (Groton) & 6900 \\
\hline SSN674 & Electric Bant (Groton) & 7000 \\
\hline SSN675 & Electric Bout (Groton) & 7000 \\
\hline SSN676 & Electric Boat (Groton) & 7000 \\
\hline SSN677 & Mare Island & 7100 \\
\hline SSN678 & Electric Boat (Groton) & 7100 \\
\hline SSN679 & Electric Boat (Groton) & 7100 \\
\hline SN680 & Ingalls & 7200 \\
\hline SNKRI & Flartrir Rnet (Fimenol) & $72 \mathrm{~m}$ \\
\hline
\end{tabular}

\author{
Comment \\ Removed from sea-going service and converted to training plaform. \\ Removed from sea-going service and converted to training platform.
}


USS CINCINNAT

USS GROTON

$\sin 093$

USS BIRM NGHAM

USS INDI

SST 697

USS BREMERTON

SSN698

USS JACKSONVILLE

SSN699

SSN700

USS IA JOLIA

SSN701

USS PHOENDX

$\operatorname{ssn702}$

USS BOSTON

SSN703

USS BALTIMORE

USS CITY OF CORPUS CHRISTI

SSN704

USS ALBUQUERQUE

SSN705

SSN706

USS MINNEAPOUIS-SAINT PAUL SSN708

USS HYMAN G. RICKOVER SSN709

USS AUGUSTA

SSN710

USS SAN FRANCISCO

USS ATLANTA

USS HOUSTON

USS NORFOLK

USS BUFFALO

USS SALT LAKE CITY

USS OLYMPIA

USS HONOLULU

USS PROVIDENCE

USS PITTSBURGH

USS CHICAGO

USS KEY WEST

USS OKLAHOMA CITY

USS LOUISVILLE

USS HEL ENA

USS OHIO

USS MICHIGAN

USS FLORIDA

USS HENRY M. JACKSON

USS ALABAMA

USS ALASKA

USS NEVADA

USS TENNESSEE

USS PENNSYLVANIA

USS WEST VIRGINIA

USS KENTUCKY

USS MARYLAND

USS NEBRASKA

SSN711

SSN712

STM713

SSN714

SSN715

SSN716

SSN717

SSN718

SSN719

SSN720

SSN721

SSN722

SSN723

SSN724

SSN725

SSBN726

SSBN727

SSBN728

SSBN729

SSBN730

SSBN731

SSBN732

SSBN733

SSBN734

SSBN735

SSBN736

SSBN737

SSBN738

SSBN739

Newpon News

Newpot News.

Electric Bout (Groton)

Electric Boat (Groton) $\quad 7900$

Electric Bont (Grotion) $\quad 7900$

Electric Boat (Growon) 7900

Electric Bout (Grotion) $\quad 8000$

Electric Boat (Groton) $\quad 8100$

Electric Boat (Groton) $\quad 8100$

Electric Boat (Groton) $\quad 8100$

Electric Bont (Groton) $\quad 8200$

Electric Boat (Groton) 8200

Electric Boat (Growan) 8200

Electric Bost (Groton) 8300

Electric Boat (Groton) 8300

Electric Boat (Groton) $\quad 8400$

Electric Boat (Groton) $\quad 8400$

Newpor News $\quad 8000$

Newpon News $\quad 8100$

Newpont News $\quad 8200$

Newpon News $\quad 8300$

Newpont News

Newport News $\quad 8300$

Newport News $\quad 8400$

Newpon News $\quad 8500$

Gectric Boet (Graton)

Gectric Boal (Grocon)

Eectic Boat (Grac

8600

Newpon News $\quad 8700$

Newpont News $\quad 8700$

Electric Boat (Groton) $\quad 8600$

Electric Boat (Groton) $\quad 8700$

Electric Bant (Growon) 8000

Electric Bant (Groton)

Glectric Bont (Groton)

Electric Boat (Grotion) $\quad 8300$

Electric Boat (Groton) $\quad 8400$

Electric Boet (Groton) $\quad 8400$

Electric Boat (Groton) 8500

Electric Boat (Grotoa) $\quad 8600$

Electric Boat (Groton) $\quad 8700$

Electric Boal (Groton)

Gectric Boat (Groton)

Electric Bont (Groton) $\quad 9000$

Electric Boat (Groton) 9100

Electric Boat (Grotion) 9300

Newpont News

8800 


\section{B. Propulsion (Navel) (Continued)}

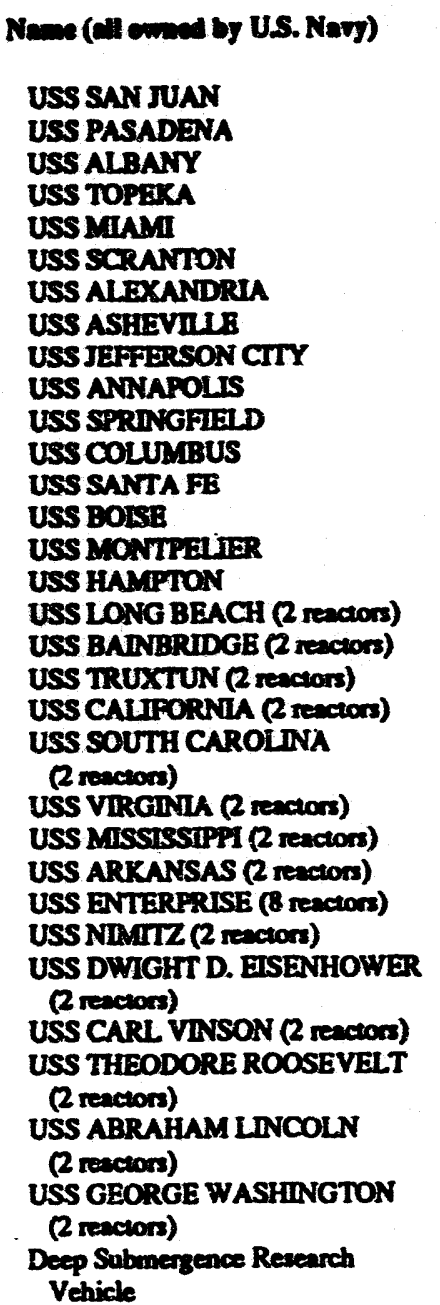

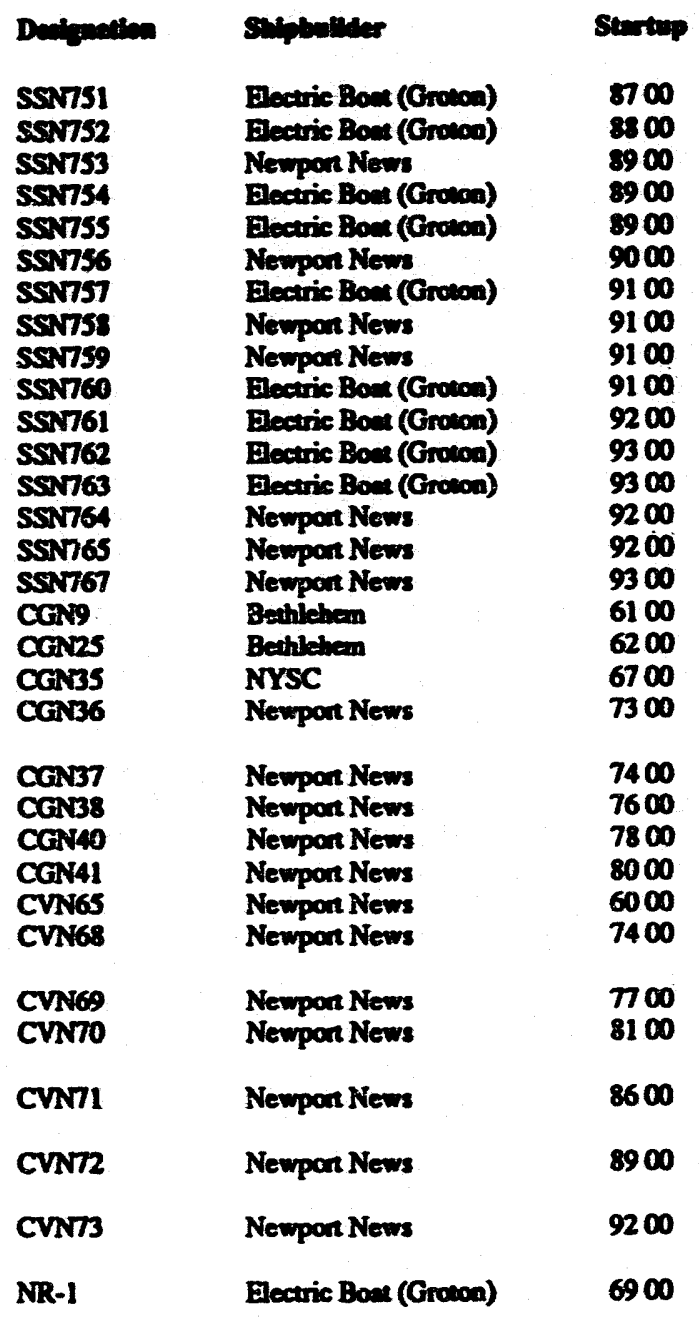




$\begin{array}{lll}\text { BEING BUILT } & & \\ \text { RHODE ISLAND } & \text { SSBN740 } & \text { Electric Boat (Groton) } \\ \text { MANNE } & \text { SSBN741 } & \text { Electric Boat (Groton) } \\ \text { WYOMING } & \text { SSBN742 } & \text { Electric Boat (Groton) } \\ \text { LOUISIANA } & \text { SSBN743 } & \text { Electric Boat (Groton) } \\ \text { CHARLOTTE } & \text { SSN766 } & \text { Newpon News } \\ \text { HARTFORD } & \text { SSN768 } & \text { Electric Boat (Groton) } \\ \text { TOLEDO } & \text { SSN769 } & \text { Newpon News } \\ \text { TUCSON } & \text { SSN770 } & \text { Newpon News } \\ \text { COLUMBIA } & \text { SSN771 } & \text { Electric Bout (Groton) } \\ \text { GREENVILLE } & \text { SSN772 } & \text { Newpon News } \\ \text { CHEYENNE } & \text { SSN773 } & \text { Newpon News } \\ \text { SEAWOLF } & \text { SSN21 } & \text { Electric Bout (Groton) } \\ \text { CONNECTICUT } & \text { SSN22 } & \text { Electric Bont (Groton) } \\ \text { JOHN C. STENNIS } & \text { CVN74 } & \text { Newpon News } \\ \text { UNTTED STATES } & \text { CVN75 } & \text { Newpon News }\end{array}$

\section{DEVELOPMENTAL POWER}

\section{A. Electric-Power Experiments and Prototypes}

(No reactors currently in this category)

\section{B. Propulsion Experiments and Prototypes}

\section{Name (Owmer). Location}

\section{OPERABLE}

Destroyer Reactor Prototype (DOE)

West Milton, NY

Large Ship Reactor Prototype (2 reactors)

(DOE). INEL Site, ID

Modifications and Additions to Reactor

Facility (DOE). West Milton, NY

Natural Circulation Test Plant (DOE)

INEL Site, ID

Trident Prototype, (DOE) West Mihon, NY

Designation $\begin{array}{r}\text { Power } \\ \text { capacit } \\ \text { net }\end{array}$

DIG

AlW

MARF

S5G

S8G

\begin{tabular}{|c|c|c|}
\hline \multirow[t]{6}{*}{$\begin{array}{l}\text { Authorized } \\
\text { power } \\
k W(t)\end{array}$} & $\begin{array}{c}\text { Inlial } \\
\text { criticallity } \\
\text { (fr mo) }\end{array}$ & $\begin{array}{l}\text { Reactor type. } \\
\text { Primelpal nuclear } \\
\text { contractor }\end{array}$ \\
\hline & 6200 & Pressurized water. GE \\
\hline & 5800 & Pressurized water. West. \\
\hline & 7600 & Pressurized water. GE \\
\hline & 6500 & Pressurized water. West. \\
\hline & $78 \propto$ & Pressurized water. GE \\
\hline
\end{tabular}

\section{TEST AND RESEARCH}

\section{A. Test}

(No reactors currendy in this category) 
B. Research

Name (Omwer). Location

\section{OPERABLE}

Amed Forces Rediobiology Research Instiwute, DNA (DOD). Requlated by NRC. Bethescin, MD Arroy Pulse Rudizion Facility. Test and

Eveluntion Command (USA). Aberdeen, MD Fast Burst Reactor Facility, Test and Evaluation Command (USA). White Sands, NM Sunionary Nenaron Radiography Syatem (USAF). MoCGellan AFB, CA

\begin{tabular}{|c|c|c|c|}
\hline ectandion & $\begin{array}{c}\text { Power } \\
\text { capacity } \\
\text { net } \\
\text { kW(e) }\end{array}$ & $\begin{array}{l}\text { Authortied } \\
\text { power } \\
\text { kW(0) }\end{array}$ & $\begin{array}{c}\text { Initial } \\
\text { critleally } \\
\text { (jr mo) }\end{array}$ \\
\hline
\end{tabular}

AFRRI

APRF

FBRF

SNRS-1

$\begin{array}{rr}1,100.0 & 6200 \\ 10.0 & 6807 \\ 10.0 & 6408 \\ 1,300.0 & 9101\end{array}$

9101

\section{Reactor type}

Principal muclear

contractor

TRIGA-Mk F. GA

Bare, fas, prompt burs. UNC

Bare, fast, prompl burst. Kaman

TRIGGA Mod Mark II. GA

\section{PART IV EXPORT REACTORS}

A. Central-Station Electric Power Plants

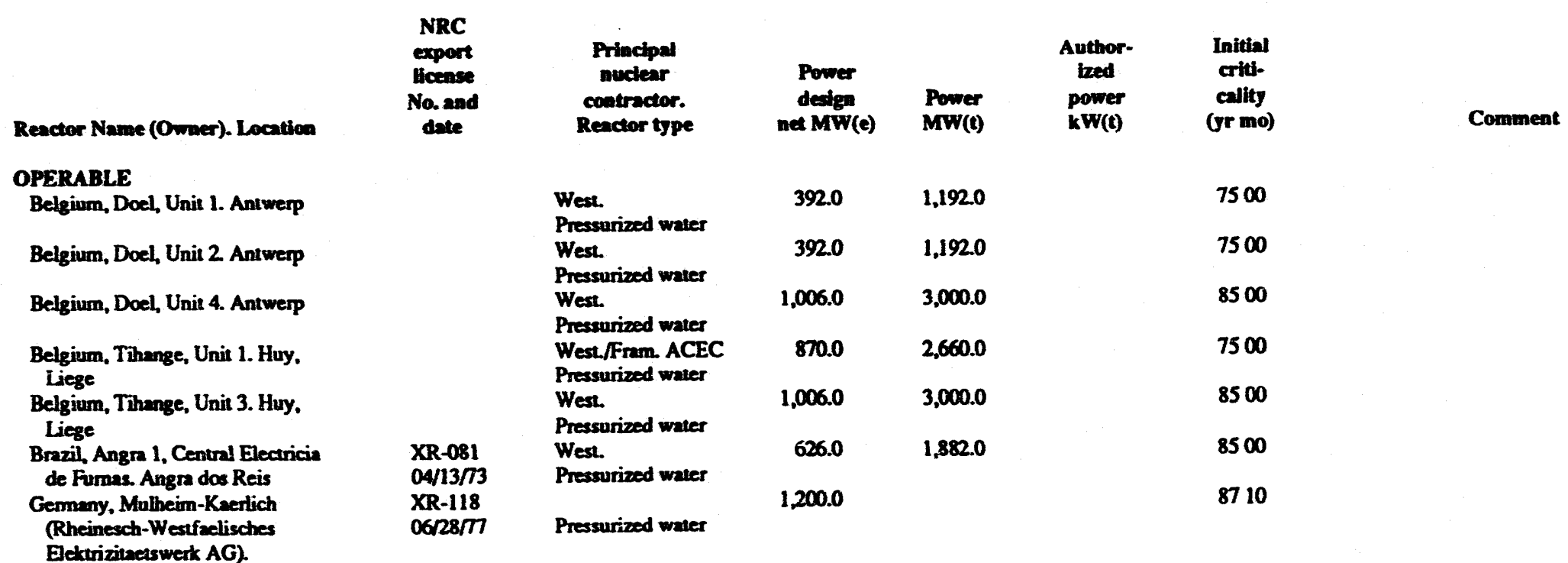




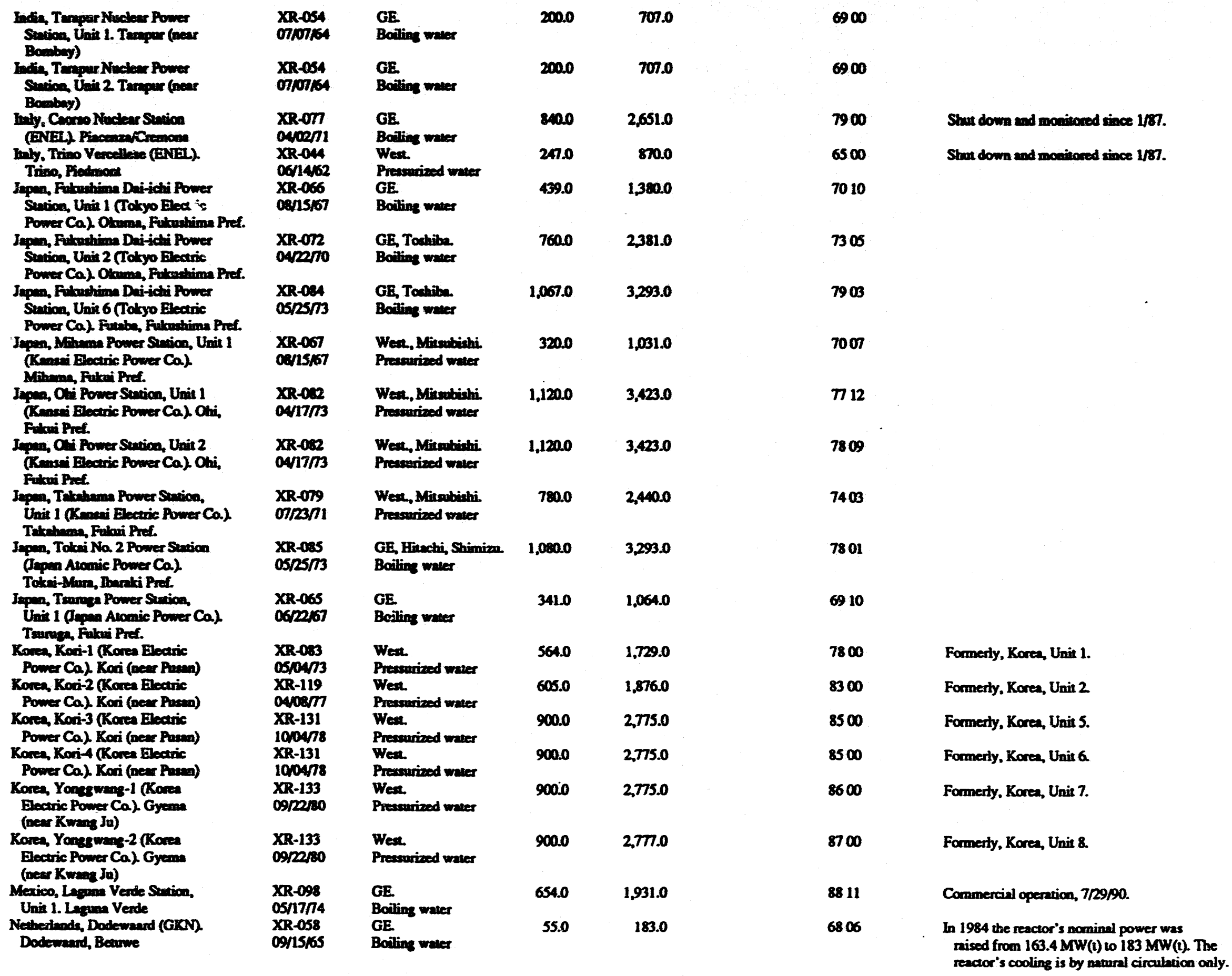


A. Centra-Station Electric Power Plants (Continued)

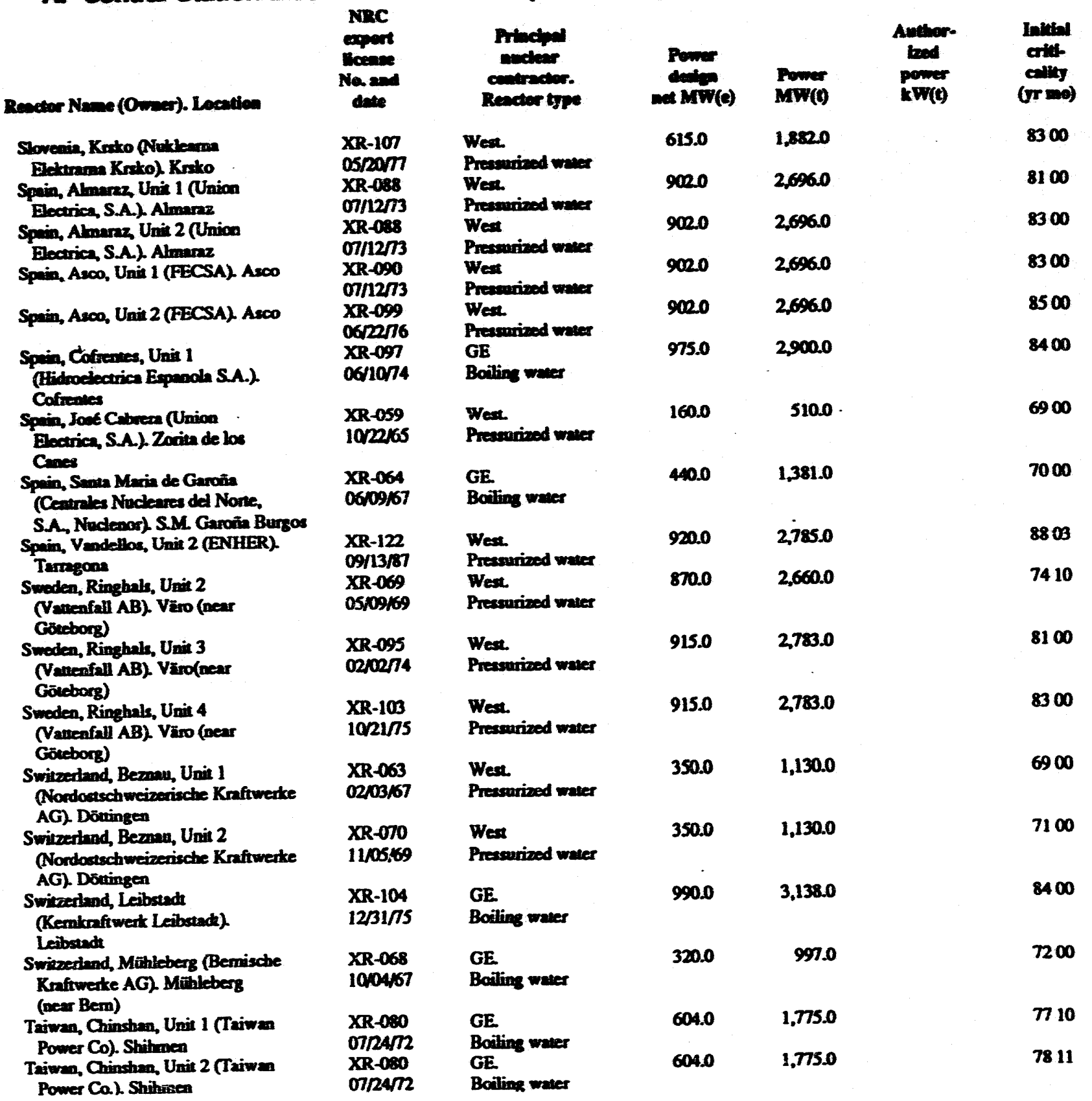


Trowea, Kro Shane, Unit 1 (Triwa Power Ca) Wanti Haim

Triven, Kro Shene, Unic 2 (Triwe

Romer Ca). Weali Haim

THuma, Manden, Unit 1 (Taiwa

Power Cal Hone chose

Trimen, Mnendera, Unie 2 (Triw

Power Ca) Howe chim

rencentr

Englend, Srawedl B (Cenerel

Eleatricity Genenting Bond).

$$
\text { sufoll }
$$

Inger Kmpiwanki-Keriwa, Unin 6

(Tatyo Enctic Power Co)

Kntiwenki, Nïgen Pref.

Inpen, Kechiwazaki-Keriwa, Unin 7

(Tokyo Dectric Power Co.).

Kentiwandri, Nïigete Pref.

Korea, Yoang weme-3 (Korea

Dlectric Power Co.). Gyeana (near

Kwane fa)

Koren, Youserwane-4 (Korea

Electric Power Ca), Gyema (near Krmane Ju)

Meriesa Laman Vende Sinion,

Unit 2 Leguna Vende

Pritipnines, Repoblic of the

Phalippine Nuclear Power Pime

Uni 1 (Notional Power Cop.)

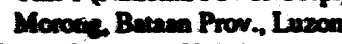

Spoin, Lemonir, Unit 1

Lemoniz

Spain, Lewoniz, Unir 2

Lemonis

Spin, Valdecabulleme, Unit 1

(HE: Sevillam de Electricidad)

Vaidecatalleoes Badajos

Spain, Valdecaballeros, Unin 2

(HE: Serilleas de Electricided)

Valdecabalieror, Badajos

\section{PLANIDD}

Korea, Ulchin 3 (Kosen Eloctis

Power Ca), Kuonganngbule-do

Kores, Ulchin 4 (Korea Electric

Power Ca). Kuoneranabulk-do

Taiven, Unit 7 (Triwen Power

Ca.) Lungerea

xis-os

0417/7

XR-096

0417/4

X:-113

cescers

Thers

ax-113

GE.

948

2,8940

8102

$\mathrm{GE}$

Boiling waver

948.0

2,8940

8203

Weat.

$890.0 \quad 2,785.0$

8403

Prosminized weter

8502

XR-148

Prestarived water

890

27250

1,1880

$3,425.0$

9400

ot/sores

Presensiced weer

GE, Toutibe.

Advenced boiline

wher

GE, Irecti.

Advenced boiling

water

XR-150

041617

Presenrived weer

1,3560

$3,930.0$

9512

$1,3560 \quad 3,9300$

9707

900

9500

9600

Comb.

9000

GE

102474

$\mathrm{xR}-120$

Boiling waver

Nex.

Preavurized water

$654.0 \quad 1,931.0$

9512

Indef.

XR-009

$07 / 12 / 73$

XR-0099

$07 / 1273$

$X R-110$

os.osm

XR-110

ospos $/ 7$

Wea.

Presearized water

West.

Presenrived water

GE.

Boiline waser

GE.

Boiling water

XR-153

040692

XR-153

040692

Comb.

Pressurized weter

Comb.

Presennized water

2825.0

$950.0 \quad 28255$

$02 / 17 / 1$

Taiwn, Unit 8 (Triwen Power

Co.) Lumenen

Ce/17/81
1,0000

1,0000
9900

hise

Inder

Indef.

Indef.

$$
\begin{aligned}
& \text { for this reactor. XR-134, }-135 \text {, and }-136 \text {. } \\
& \text { Only the vendor who wins the contrnat will } \\
& \text { be allowed to use its license. } \\
& \text { The NRC has issued three licenses to vendors } \\
& \text { for this reactor. XR-134, }-135 \text {, and }-136 \text {. } \\
& \text { Onty the vendor who wins the contract will } \\
& \text { be allowed to use its license. }
\end{aligned}
$$

The NRC has issued three hocenes to vendors 


\section{B. Propulalon}

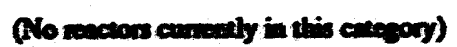

\section{TEST, RESEARCH, AND TEACHMG}

\section{A. Ceneral Irradlation Teet}

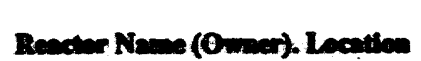

Orsanes:

Jye, JRR-2 (Japen Alowic Energy

Donki Pref.

Inper, NSRR (Inpea Aromic Energy

Benali Pref.

Netherinand (Eneray Center).

Prom

Romania (Taxime for Nucliear

Reseanch), Buctureas

Romemia (bestimte for Nuciear

Revearch). Buchares

Somb Africa, Safari-1 (Alomic

Evergy Bound). Pelindabe (near Prearii)

Sweden (Sudsvit Energitelonik).

Smodovil

\section{B. Ceneral Research}

\section{OTrRable}

Avetralin, Mouts (Alomic Energ) Commisrion) Lucas Heighis, New South Wales

Anstrin, Astro (Seibersodorf

Research Center). Seibersdoff Bengledesh (Instime of Nuclear Technoloyy). Dhalo

Colombin, IAN-R1 (institure of

Colombia, IAN-R1 (Institur

Denenet. DR-1 (Rieó Naican

Lebormeny). Rito

Expland (lmperial Chemical

industries). Billinghn, Teeside

NRC

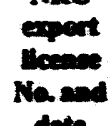

XR-015 1011157

AMF.

XR-101

1016/74

GA.

XR-017

01/1758

XR-091

06297

XR-091

062973

XR-042

$06 / 14 / 51$

XR-019

05/1458

GA

GA.

XR-039

0\%12א0

XR-023

090358

$x \mathrm{x}-126$

XR-126

$10 / 05 / 82$

XR-053

05127164

XR-005

0404/57

XR-074

032371
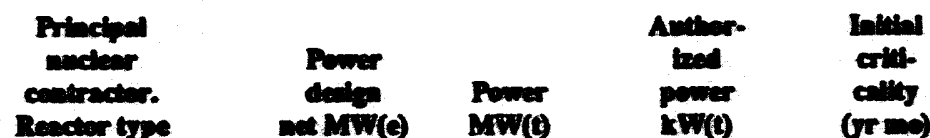

Heavy waer, CP-5

TRIGA-ACPR

AC. Tank (MTR)

TRIGA-ACPR

TRIGA (MPR 10)

AC. Tank

AC Tank (MTr)

10,0000

6010

3000

7506

45,0000

6109

500.0

7900

14,000007900

$20,000.0 \quad 6500$

$50,000.0 \quad 6000$
AR. UTR-10

AMF. Pool

GA.

TRIGA-Mk II

Locitheed. Pool

A. L-5S

GA. TRIGA-Mat
15.0

6100

$5,000.0 \quad 6000$

$3.000 .0 \quad 8600$

$20.0 \quad 6500$

$2.0 \quad 5700$
$2500 \quad 7100$
1985 the reacior vesced was replaced. If is now refurbisted. Manuf meturer of the vesed: Royal Schelde of Fleshing (Vliasingen, Hollend). 
2500

7900
Commistion). Nahal Soreq
AMF. Pool

GA. TRIGA-ME II

Research reactor

GA. TRIGA-MK II

GA. TRIGA-MK II

GA. TRIGA-M II

GA. TRIGA-ME III

AMF. Pool

GA. TRIGA

Conversion

AMF. Pool

GA. TRIGA-MA II

GE. Pool

ORNL Pool

Conversion

GA. TRIGA-MR II
Isroel (Atomic Energy

coly (halina Agency for $\mathrm{New}$

Technology, Energy and the

Enviroment). Rome

Jamaica (Kingston) Kingston

Korea (Advancod Energy Research

Institure). Seout

Korea (Advanced Energy Research

Instinue) Sooul

Mslaysia (Tun Ismail Alomic

Research Centre). Kuala Lumpur

Mexioo (National Commission for

Nuclear Energy). Salazar

Patistan, PARR (Alomic Energi

Commission). Istamabed

Philippines, Republic of the, PRR-1

(Philippine Noclear Research Instivuse)

Quezon City

Portugal, RP-1 (National

Laboratory of Engineering and

Industrial Technology). Sacaven

Slowenia (Josef Siefm Nuclear

Institute) Ljubljana

Spain (Nuclear Energy Boand-JEN).

Madrid

Switzerland (Panl Scherrer

Institure). Wuerenlingen

Thailund, TRR-1 (Office of Alomic

Energy for Peace). Bankok

09/13/57

XR-055

$01 / 30164$

XR-010

07/29/57

XR-112

0505/77

5,0000

1,0000

6000

6000

250.0

20000

6200

$\mathbf{7 2 0 0}$

$10000 \quad 8200$

$1.0000 \quad 6800$

50000

3,0000

$1,000.0$

6100

5000

6600

$3,000.0$

10,0000

2,0000

$1,000.0$

6200

Com (Atomic Energy

Turkey (Technical University of

Istrmbul). Istanbul
XR-030

09,04/59

XR-108

03/2476

\section{AMF. Pool}

GA. TRIGA-MR II

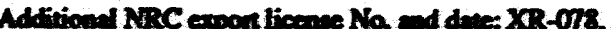
S20071.

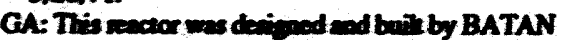

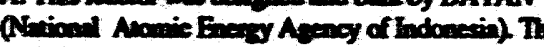
dexion was bed on the dexipe of TRGA Man II reacor, wiht meringm power level of $250 \mathrm{~kW}$. In 1979

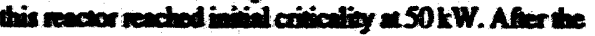

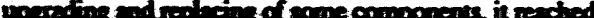

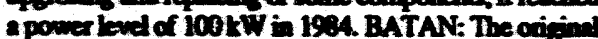

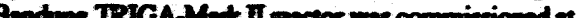
250tw() in 1964. $\mathrm{k}$ wes upended and reached a pomer

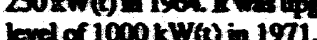

Design power: $10 w$.

The original Philippine Research Reactor (PRR-1)

was designed and built by GE and was commiscioned as a $1 \mathrm{MW}$ reactor in 1963. The reactor was shut down in 1/85 for exiensive upgrading and has now become a TRIGA Conversion. h has a power level of $3 \mathrm{MW}$ and reached criticaliny on 311/88.

\section{This is the 1955 Geneve conference reactor} rebuilt with increased power.

The Thei research reactor, TRR-1, buin by Curriss-Wrigh and started up in 1962, ociginally operated at $1000 \mathrm{~kW}(\mathrm{l})$. In 6775 the TRR-1 was thot down for conversion to TRR-1M1, a TRIGA-Mark III system adpred for pool inenallation. The TRR-1M1, with a power level of $2000 \mathrm{~kW}(t) / 2000 \mathrm{MW}$ pulsing was commissioned 11/77. 


\section{TEST, RESEARCH, AND TEACHMG}

\section{B. General Pesearch (Continued)}

\begin{tabular}{|c|c|c|c|c|c|}
\hline Reacter Name (Omber), Locention & $\begin{array}{c}\text { NRC } \\
\text { expert } \\
\text { Naseane } \\
\text { Nande }\end{array}$ & 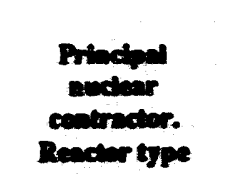 & 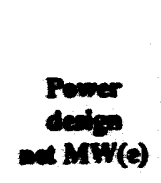 & $\begin{array}{l}\text { Puwar } \\
\text { MWW(t) }\end{array}$ & 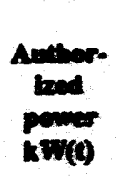 \\
\hline \multicolumn{6}{|l|}{ OPERABLE (Comimand) } \\
\hline $\begin{array}{l}\text { Venemel (Instime for } \\
\text { Scientific Reseanch). Curncas } \\
\text { Znire (Regional Cencer for }\end{array}$ & $\begin{array}{l}\text { XR-018 } \\
01 / 16558\end{array}$ & $\begin{array}{l}\text { GE. Pool } \\
\text { GA. TRIGA-MIII }\end{array}$ & & & $\begin{array}{l}3,000.0 \\
1,000.0\end{array}$ \\
\hline $\begin{array}{l}\text { BEING BUILT } \\
\text { Morocoso (CE.N., Meimon) } \\
\text { Rebee }\end{array}$ & $\begin{array}{l}\text { XR-158 } \\
1025 / 91\end{array}$ & GA. TRIGA-MK II & & & $3,000.0$ \\
\hline $\begin{array}{l}\text { PLANNEDD } \\
\text { Albania. Tirama }\end{array}$ & $\begin{array}{l}\text { XR-154 } \\
\text { Pendias }\end{array}$ & GA. TRIGA-MAR I & & & 250.0 \\
\hline
\end{tabular}

\section{University Research and Teaching}

\section{OPERABLE}

Austria (Vienena Polytechnic

Instivued Vienat

XR-035

Instivuc). Vienas

Genis). Belo Horizonte

Brazil (University of S50 Paralo).

Sío Paulo

$11 / 2459$

XR.028

08103159

$\mathrm{XR}-002$

$01 / 2257$

XR-011

$08 / 27 / 57$

Hamilkon, Ontrio

China, Republic of Nrtional

Tsing-Hua Universiry). Hsinch

Finland Institute of

Techrology). Helsinki

$\mathrm{XR}-020$

$06105 / 58$

XR-040

$0405 / 61$

Germany (Instime for Nuclear

Modicine). Heidelbers

XR-060

$02 / 14 / 66$

GA. TRIGA-Mk II

GA. TRIGA-MK I

Bew. Pool

AMF. Pool

GE. Pool

GA. TRIGA-MK II

GA. TRIGA-Mk I

Germany (Johannes Gruenberg

University of Mainz). Mainz

Germany (Medical Colinge of

Hanover). Hanover

Germany, FRM Gensching (Technical

University of Munich). Monich
XR-050

0411/64

XR-076

$0226 / 71$

XR-004

03/15/57
GA. TRIGA-M II

100.0

6500

GA. TRIGA-Mk 1

AMF. Pool

$250.0 \quad 7300$

$4.000 .0 \quad 5700$
250.0

6200

100.0

6000

PART N EXPORT REACTORS

$5.000 .0 \quad 5700$

$5,000.0 \quad 5900$

$1,000.0 \quad 6200$

$250.0 \quad 6200$

$250.0 \quad 6600$

This TRIGA-Mk I reactor was installed in 1966. In 1977, the reactor was shut down, dismantied, and moved to mother up again in 1978. This operation was referred 20 as "TRIGA I" and "TRIGA II" brilding. After this move, it was sented

\section{This TRIGA reactor opented at the 1958 International Conference in Geneve prior to ship ment to the University of Lovaniun in 659. I is the firt reactor wo be opented on the Africen continent.}

Originally planed as TRIGA-MkI, resctor was upgnded to TRIGA-MR II. Original expont bicenes was not used.

Expon ticense explicuion dued 109yo. 


\begin{tabular}{|c|}
\hline 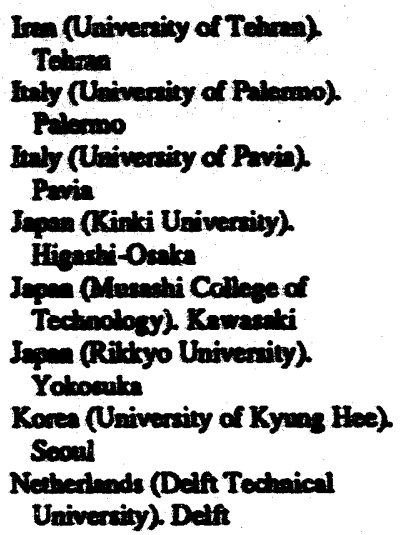 \\
\hline
\end{tabular}

Switwertand (Univeniiny of

Bacel). Based

\section{AMF. Pool}

AGN. $201-110$

GA. TrGA-AM I

AR. UTR-10

GA. TRIGA-MA II

GA. TRIGA-MK II

AON. 201

AMF. Pool (Mm)

AGN. 211-100
5,0000

\section{0}

6000

$2 n o$

6500

6111

100.0

100.0

6301

6112

8200

20000

630

5800

Fued expriter being souphe.

Noeligitis power. Shen down for renewal of coserting beence.

Shut down for resewal of openting license.

Neqligible power.

Nepligible poner.

The Netherinds resenrch neactor was originally opereded at the Ametendan Imeenational Erhibition in 657; mejor portions of the exhibition reactor system were used to fabricate the present resctoc.

This rewctor was opented in the Intemntional Science Section of the Brusucls Irformation Exhibition, 4/15/58 to 101/58, prior to tranafer to the University of Basel. Neglipible power.

PART V CRITICAL ASSEMBLIES

\section{CMMLIAN}

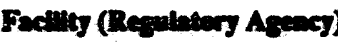

Advenced Ten Reactor Critical Fecitity (DOE)

Argane National Laborationy, Ideho

Division (DOE)

Los Almos National Laboratory (DOE)

Los Alamos National Lebormeny (DOE) Los Alemos Netional Leboretory (DOE) Los Alemos Netional Leboretory (DOE) Los Almos Nasional Labortory (DOE) Los Almons National Laboretory (DOE) Los Almos National Laboretory (DOE) Los Almos National Leboctiony (DOE) Los Akmos National Laboestory (DOE)

Rensucleer Pobrochnic Inotive (NRC)

SNL Critical Assembly (DOB)

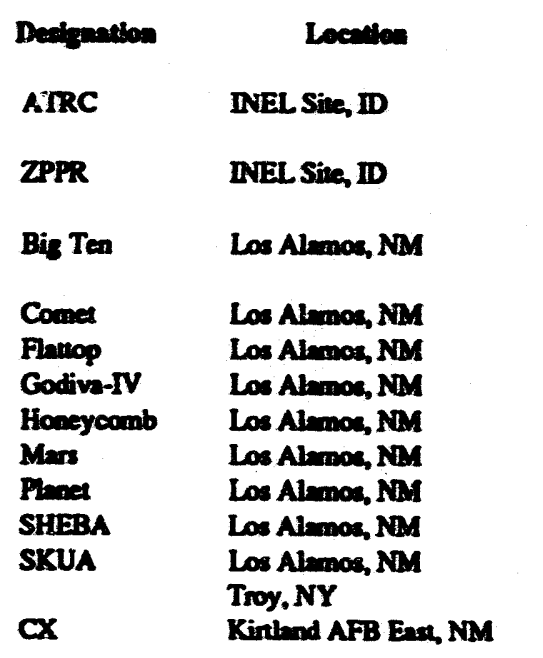

Abbreviation

ATRC

ANL-IDAHO

LANL, Kiva II

LAN Kive II

LANL, Kiva II

LANL, Kiva II

LANL Kiva I

LAN Kive I

LANL Kiva II

LANL, KivaI

LANL, Kiva III

Rensediser

Sendia

\section{Comment}

ATR physics, core-loading and core-design meneurements.

To be shue down FY95.

$U(10)$-metel cylinder in thick metal

reflector.

Critical-configurnion safery and neutronic tests.

Spherical metal cores in thick metal reflector.

Fast neutron imadiation, pulse capability.

Flexible split table assembly.

Verical rable assembly machine.

Vervical uble assembly.

Solution high exergy burst assembly.

Fass nevtron irrediation, pulse capebility.

Crivical experiment assembly.

Spece power neutronics. 


\section{PART V CRITICAL ASSEMBLIES}

\begin{tabular}{|c|c|c|c|c|c|c|c|}
\hline \multirow{3}{*}{ 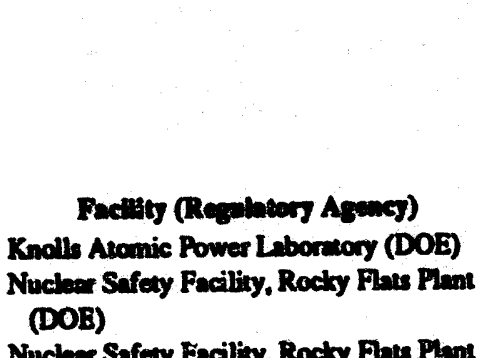 } & \multirow{3}{*}{$\begin{array}{l}\text { Dedonation } \\
\text { FCPE } \\
\text { Horizontaly } \\
\text { Split Table }\end{array}$} & & \multicolumn{2}{|c|}{ Equipment } & & \multirow[b]{2}{*}{$\begin{array}{l}\text { Inidial } \\
\text { criticallty } \\
\text { (r mos) } \\
7000 \\
6500\end{array}$} & \multirow[b]{2}{*}{$\begin{array}{l}\text { Comment } \\
\text { Full core physias experiment. } \\
\text { Critical-configunation safery tests }\end{array}$} \\
\hline & & $\begin{array}{l}\text { Locadien } \\
\text { Schiemecendy, NY } \\
\text { Golden, CO }\end{array}$ & $\begin{array}{c}\text { No. } \\
\text { of } \\
\text { cello } \\
2\end{array}$ & $\begin{array}{c}\text { Na. of } \\
\text { control } \\
\text { pantel } \\
1 \\
1\end{array}$ & $\begin{array}{l}\text { Abloreviadion } \\
\text { KAPL } \\
\text { RPP-NSF }\end{array}$ & & \\
\hline & & Golden, CO & 1 & 1 & RFP-NSF & 6500 & \\
\hline
\end{tabular}




\section{REACTORS AND FACILITIES SHUTDOWN OR DISMANTLED}




\section{POWER REACtORS}

\section{PART I CIVILIAN REACTORS (DOMESTIC)}

\section{A. Central-Station Electric Power Plants}

\section{Name (licensee) [docket number] \\ Boiling Nuclear Superheater Powes Station (AEC and Puerto Rico \\ Water Resources Authority) \\ Carolinas-Virginia Tube Reactor \\ (Carolinas-Virginia Nuctem \\ Power Associntes, inc.)}

Dresden Nuclear Power Station, Unit 1 (Commonwealth Edison Ca.) [50-010]

Elk River Reactor (AEC and Rurd

Cocperative Power Association)

Enrico Fermi Atomic Power Plam, Unit (Power Reactor Development Ca) [50-16]

Fort St. Vrain Nuclear Genenting

Station (Public Service Company of Colondo) [50-267]

Hallam Nuclear Power Facility.

Sheldon Station (AEC and Consumers

Public Power District)

Humbolde Bay Power Plane, Unir 3 (Pacific Gas \& Electric Ca) [50-133] Indian Point Stration, Unit 1

(Consolidared Edison Co. of

New York, Inc.) [50-3]

La Crosse (Genoa) Nuclear Generating Station (Dairyland Power Cooperative) [50-409]

Pathinder Alomic Plant (Nonthem

States Power Ca.)

\begin{tabular}{|c|c|c|c|c|c|c|c|}
\hline Locadion & $\begin{array}{l}\text { Princlpal } \\
\text { nuclear } \\
\text { contractor }\end{array}$ & Type & $\begin{array}{c}\text { Power } \\
\text { MD capacity } \\
\text { net MW(e) }\end{array}$ & $\begin{array}{l}\text { Power } \\
\text { lleensed } \\
\text { MW(I) }\end{array}$ & $\begin{array}{c}\text { Inlitial } \\
\text { criticallty } \\
\text { (rr mo) }\end{array}$ & $\begin{array}{l}\text { Shut down } \\
\text { peresanently } \\
\text { (Jr mo) }\end{array}$ & Cosmmeat \\
\hline Punta Higuern, PR & Comb. & $\begin{array}{l}\text { Boiling water, } \\
\text { integral nuclear } \\
\text { superheat }\end{array}$ & 16.5 & 50.0 & 6400 & 6800 & \\
\hline Parr, SC & Weat. & $\begin{array}{l}\text { Pressure tube, } \\
\text { heavy water }\end{array}$ & 17.0 & 64.0 & 6300 & 6701 & $\begin{array}{l}\text { The last CVTR shudown occurred } \\
\text { 1/2467. A license amendment } \\
\text { issued 6/14/67 authorizes CVNPA } \\
\text { to possess but not operate the CVIR. }\end{array}$ \\
\hline Morris, Il & $\mathbf{G E}$ & Boiling water & 200.0 & 700.0 & 5900 & 7810 & \\
\hline Elk River, MN & $A C$ & Boiling water & 220 & 58.2 & 6200 & 6802 & $\begin{array}{l}\text { The Elk River Reactor was shut down doe } \\
\text { to technical problems in February 1968; } \\
\text { in 1974, dismantling and removal of } \\
\text { this facility was completed. }\end{array}$ \\
\hline Lagoona Beach, MI & PRDC & $\begin{array}{l}\text { Sodium cooled, } \\
\text { fast }\end{array}$ & 60.9 & 200.0 & 6300 & 7209 & \\
\hline Platteville, CO & $\mathbf{G A}$ & $\begin{array}{l}\text { High temperature, } \\
\text { gas cooled }\end{array}$ & 330.0 & 842.0 & 7401 & 8908 & \\
\hline Hallam, NE & AI & Sodiurn graphite & 75.0 & 240.0 & 6200 & 6409 & $\begin{array}{l}\text { The Hallam Nuclear Power Facility } \\
\text { was shut down in September } 1964 \\
\text { due to moderntor-can failures. } \\
\text { Entombment of the reactor was } \\
\text { completed in } 1968 \text {. }\end{array}$ \\
\hline Eurekn, CA & GE & Boiling water & 65.0 & 242.0 & 6300 & 7607 & \\
\hline Buchanan, NY & BaW & $\begin{array}{l}\text { Pressurized } \\
\text { water }\end{array}$ & 265.0 & 615.0 & 6200 & 7410 & $\begin{array}{l}\text { In the Consolidated Edison Indian } \\
\text { Point Station, the } 615 \mathrm{MW}(\mathrm{t}) \text { was } \\
\text { increased by an cil-fired superheaier } \\
\text { to produce } 265 \mathrm{MW}(\mathrm{e}) \text { net. }\end{array}$ \\
\hline La Croser, W & AC & Boiling water & 48.0 & 165.0 & 6707 & 8704 & \\
\hline Sioux Falls, SD & $\mathbf{A C}$ & Boiling water & 58.5 & 190.0 & 6400 & 6709 & $\begin{array}{l}\text { The Pathfinder Plant has been shut down } \\
\text { since Novermber 1967. On Sept. 9, 1968, } \\
\text { Northem States Power Company } \\
\text { amnounced plans to instull gas-fired boilers } \\
\text { for operation the summer of } 1969 \text {. }\end{array}$ \\
\hline
\end{tabular}




\section{POWER REACTORS}

\section{A. Central Station Electric Powrer Plants (Continued)}

\section{Name (licensee) \\ [decket number]}

Piqua Nuclear Power Facility (AEC and City of Piqua)

Rancho Seco Nuclear Generating Station, Unit 1 (Sacramento Municipal Uaility District) [50-312]

San Onofre Nuclear Genenting Station. Unit 1 (Southem Califomia Edison) [50-206]

Shippingpon Alomic Power Station (DOE and Duquesne Light $\mathrm{Co}_{0}$ )

Shorcham Nuclear Power Station

(Long Island Lighting Co.) [50-322]

Three Mile Island Nuclear Station, Unit 2

(GPU Nuclear Corp.) [50-320]

Trojan Nuclear Plant, Unit 1 (Portland

General Electric Co.) [50-344]

Yankee Nuclear Power Station (Yankee

Atomic Electric Co.) [50-029]

\section{B. Dual-Purpose Plants}

Name (Owner)

N Reactor (DOE)

\begin{tabular}{|c|c|c|c|}
\hline Location & $\begin{array}{l}\text { Princlpal } \\
\text { Duclear } \\
\text { contractor }\end{array}$ & Type & $\begin{array}{c}\text { Power } \\
\text { MD capacte } \\
\text { net MW(e) }\end{array}$ \\
\hline Piqua, $\mathrm{OH}$ & $\mathbf{A I}$ & $\begin{array}{l}\text { Onganic cooled } \\
\text { and moderated }\end{array}$ & 11.4 \\
\hline Clay Stution, CA & BaW & $\begin{array}{l}\text { Pressurized } \\
\text { water }\end{array}$ & 873.0 \\
\hline Sen Clemente, CA & West. & $\begin{array}{l}\text { Pressurized } \\
\text { water }\end{array}$ & 436.0 \\
\hline Shippingport, PA & West. & $\begin{array}{l}\text { Pressurized } \\
\text { water }\end{array}$ & 60.0 \\
\hline
\end{tabular}

Brookhaven, NY

isiddletown, PA

GE

Prescolt, OR

Rowe, MA

West.

West.

\section{Boiling water}

Pressurized

$$
\text { waler }
$$

Pressurized

water

water

Principal

nuclear

Location

Hanford Site, WA contractor

WHC
Type

Graphite

\section{PART I CIVILIAN REACTORS (DOMESTIC)}

820.0

906.0

1075.0

167.0

Power Inithal Shut dow

MW(t) (jr mo) (jrmo)

455

6300

6600

$2,772.0 \quad 7409 \quad 8906$

$1,347.0 \quad 6706 \quad 9211$

$236.0 \quad 5700 \quad 8210$

$236.0 \quad 5700$

$\begin{array}{rrr}2,436.0 & 8502 & 9107 \\ 2,772.0 & 7800 & 7900 \\ 3411.0 & 7512 & 9300 \\ 600.0 & 6008 & 9202\end{array}$

Power capscity net MW(e)

860.0
Power

licensed

$\mathbf{M W}(t)$

4000.0
Initial criticality Shut down (Jr mo) (Jr mo)

6312

9100

\author{
Comment
}

The dismantemert program for the Pique Nuclear Power Facility was completed in February 1969. Possession only license, $3 / 92$.

Possession only license is contingent as of 1092 on the fuel being removed.

The first core for the Shippingport station began power operation in 1957 with a capacity reting of $60 \mathrm{MW}(\mathrm{e})$. The second core began power operaion in 1965 with a capecity raing of $90 \mathrm{MW}(\mathrm{e})$.

The thind core, a light water breeder

reactor (LWBR), began power operecac in 1977 with capacity rating of con in 197 (e) With a capacty rating of $60 \mathrm{MW}(\mathrm{e})$. Owned by the Department of Energy, the reactor plant was shut down on Oct 1, 1982. Defueling was completed in September 1984. Decommissioning was completed in December 1989, and the site was released for use without any restrictions. Possession only license, 7/20/91.

Three Mile Island Nuclear Station, Unit 2 has been shut down since the $3 / 28 / 79$ accident. Core removal is finished. Possession only license, 5/5/93.

Licensee announced permanent shutdown 2/92. Possession coly license, 8/92

Comment

Reactor, a DOE-owned reactor for production of special nuclear materials, also produced steam that was supplied to m adjacent electric generating plant, owned and operated by Washington Public Power Supply System. The reactor has been directed to transition from stundby to the termination mode in preparation for decommissioning. 


\section{Propulston (Maritime)}

\begin{tabular}{|c|c|c|c|c|c|c|c|}
\hline Name mander ander & Nocloar dealener & Sulpbailder & Type & 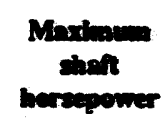 & 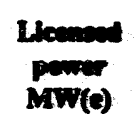 & $\begin{array}{l}\text { sturt. } \\
\text { (or mo) }\end{array}$ & 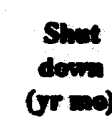 \\
\hline Nuctear Stip SA VANNAH & Bey & NYsC & Promarived & 22,000 & & 6100 & \\
\hline
\end{tabular}

\section{EXPERMENTAL POWEAREACTOR SYSTEMS}

\section{A. Electric-Power Systems}

\section{Name (Regolatory agency). Lecenter}

Bailing Reactor Experiment
No. 1 (BOA INEI Site, ID

\section{Boting Recotor Expeciment}

Na. 5 (BOA) DEIL Site, ID

Boiling Recctor Experiments

(DOE), INEL, Site, ID

SSADA Vallecitos Experiment Superteat Reactor (NRC)

Pleasanton, CA

\section{Experimental Beryllium Oxide}

Reactor (DOE). INEL Site, ID

\section{Experimented Boiling Water} Reactor (DOE). Argonne, II
Pewror canacing net iniv(e)

kW(i)

\section{Altwortion Power}

pever Mivespadt

Power Inlital $\operatorname{MW}(0)$ (jr wo) (rome) $1,400.0$

26000

$20,000.0$

$2,400.0$

$15,500.0$

54.00

5806

5300

$6200 \quad 6400$

Boiling water.

$17,000.0$

6300

6702

$10,000.0$

$4,000.0$

$100,000.0$

6300

5600

6705

\section{EVESR. Light-} ANL.

\section{Dexis. Type. Primelpal nect contr.}

Comment

BORAX-1

Boiling

water. ANL

BORAX-S

Boiling water,

integral

nuclear super-

heat. ANL

BORAX-2,-3,-4. This facility was originally built and operated

water mod-

erated, super-

heater. GE

EBOR. Gas cooled, The EBOR reactor experiment was terminated

GA

EBWR. Boiling water. ANL No. 2 (BORAX-2). With the addition of a turbogenerator, it operated during 1955 as BORAX-3 and on 7/17/55 produced sufficient electricity to light and power Archo, ID $\rightarrow$ U.S. first. BORAX-4, a further modification, operated from 12/56 to 6/58, when the experiment was shut down.

Owner. Empire States Atomic Development Associates and General Electric Company. in December 1966 prior to completion of construction.

The EBWR achieved $100,000 \mathrm{~kW}(\mathrm{t}) 11 / 11 / 62$ Operation of EBWR in the Boiling Water Program was closed out 12/62. The reactor was used in suppor of the Plutonium Recycle Program and anained criticality using plutonium as its principal fuel 9/22/65. In suppon of that program, it operated at power levels as high as $70,000 \mathrm{~kW}(\mathrm{l})$. Operation in that program was completed 6/67. 


\section{EXPERIMENTAL POWER-REACTOR SYSTEMS}

\section{A. Electric-Power Syetems (Continued)}

\section{Nasoe (Regohtary rogency). Locatica}

Experimenel Breeder Renctos No. 1 (DOE). INEL Sine, ID

\section{Experinented Gas Cooled}

Reactor (DOE), Onk Ridge, TN

\section{Heavy Waner Components Test} Reactor (DOE). SRL, Aiken, SC

Homogeneous Reactor Experiment
No. 1 (DOE), Oak Ridge, TN
Homogeneous Reactor Experiment
No. 2 (DOE), Oak Ridge, TN
Los Almmos Molven Plutonium
Reactor Experiment (DOE).
Los Alamos, NM
Los Almos Power Reactor
Experimens No. 1 (DOE).
Los Alamos, NM
Los Alamos Power Reactor
Experimeat No. 2 (DOE).
Los Alamos, NM

Molven Seh Reacior Experiment (DOE). Oat Ridge, TN
$21,900.0 \quad 84,300.0$

$40,000.0$

$61,100.0$

$140.0 \quad 1,000.0$

300.0

$5,200.0$

$1,000.0$

$2,000.0$

$1,000.0$

$8,000.0$

\section{PART I CIVILIAN REACTORS (DOMESTIC)}

Dese. Trpe.
Principal
nuch contr.

EBR-1. NaK-ooled, fast. ANL

EGCR. Gas cooled arophise mod ernted. KE-AC

EOCR Organic cooled and moderaled. Flour-AI HWCTR. Pressurized heavy water. Du Pont

HRE-1. Aqueous

homogeneous solution $\left(\mathrm{UO}_{2} \mathrm{SO}_{4}\right)$ ORNL. HRE-2 Aqueous hamogeneous solution $\left(\mathrm{UO}_{2} \mathrm{SO}_{4}\right)$ ORNL

LAMPRE-1. Fast

molien pluanium fueled, sodium cooled. LANL LAPRE-1. Aqueous hamogeneous (phosphoric scid). LANL

LAPRE-2 Aquecus

homogeneouss

(phosphoric scid).

LANL

MSRE. Single

region, grophite moderued. ORNL
In a trial run 12/21/51 and 12/22/51, EBR-1 generuted the word's firs electic power from nuclear energy and was first to demonetrente. in 7/53, the feasibility of breeding and the compatibility with breeding economy of compatibity with breeding cocaciny of sodium-potassium alloy as a biquid-metnl columt. If operated with a plutonium-bear reactor was docommissioned and dismanted earty in 1964. The facility was dedicated as a historic lendent 826\%6. If is cpen to the public from Memorivl Day weekend to Labor Day weekend, anmally.

The EGCR project was terminited 1/66 prior to the completion of construction.

EOCR construction was terminated 12/62. The facility was mothballed prior to cperation. 


\section{Orgmic Medernted Renctor \\ Experiment (DOE). INEL Site, II}

Resctor (DOE), Rictimen, WA

$70,000.0$

5700

6300

$3,000.0$

$23,500.0$

Reactor Project (DOE).

Sertion, PA

Sodium Reactor Experimen

(DOE). Sanes Susana, CA

Soumwen Experimental Fast Oxide

Reactor (NRC). Strictler, AR

Uhe High Tempernture Reactor Ex-

periment (DOE). Los Almmos, NM

Vallocies Boiling Water Reactor

(NRC). Pleasintion, CA
20,0000

$3,000.0$

$5,000.0 \quad 33,000.0$
6000

6900
Phwoniunn Recycle Text

\section{B. Space Nuclear Auxlllary Power (SNAP)}

\section{Name (Regulatory agency).} Location

SNAP-02 Developmentel System

(DOE). Santa Susam, CA

SNAP-02 Experimental Reacto

(DOE). Santa Susarna, CA

SNAP-02/10A TSF Shielding

SNAP-08 Developmental Reacto

(DOE). Santa Susana, CA

SNAP-08 Experimental Reactor

(DOE). Sunta Susma, CA

SNAP-10A Flight System (DOE)

In orbie, US
Experiment (DOE). Oak Ridge, TN

\section{Power \\ capacity \\ Power
licensed
$k W(\theta)$ \\ Authortad
power \\ Power
MB eapucity
net MW(e)}

Power

Initial

Smit

(pr mo) $(r \mathrm{mo})$

500

6100

6300

50.0

5900

6000

100

6700

7300

600.0

680

6900

600.0

6200

0.5

39.0

6500

6500

6500

0.5

39.0

(Spare)

Onk Ridge, TN

SNAP-10A Flighe System

Ground Test Na. 1 (DOE)

SNAP-10A Flight System

Ground Test No. 3 (DOE)

Senta Susema, CA cooled and

modernted AI

PRTR. Pressure

mbe, hervy water

modemed and

cooled. WHC

No Deag.

Pressurized

water. Weat

SRE Sodium

eraphite. AI

OMRE demonetrated the tectunical and economic feacibitity of wing liquid tydrocarbon

serpheayls as coolent and/or moderitor.

Owner: Sextion Nuclear Experimental Corp.

SRE cpessed a $20 \mathrm{MW}(t)$ until stur down

2/64 formodification to permit an increase in power

level to $30 \mathrm{MW}(\mathrm{t})$. On 12/2/66, deactivation was announced. Ownens: DOE and Southem Californin Edison Ca.

SEFOR. Sodium

cooled, fast. GE

UHIREX. Helinm

cooled. LANL

VBWR. Boiling

wher. GE

\section{Owner: Gesenal Electric Company and Pacific}

Gas \& Electric Co.

\section{Designation. \\ Type. Princtpal muclear \\ contractor \\ S2DS. NaK-cooled \\ AI \\ SER. NaK-cooled \\ AI \\ SNAP-TSF. \\ NaK-cooled \\ AI-ORNL \\ S8DR. NaK-cooled. \\ AI \\ S8ER. NaK-cooled \\ AI \\ S1OPS-4. \\ NaK-cooled. AI}

S1OFS-5.

NaK-cooled. AI

S1OFS-1.

NaK-cooled. AI

S10FS-3.

NaK-cooled. AI
S1OFS-4 operated in orbit April-May 1965.

Operation terminated unexpectedly after 43 days al power, probably owing to a sequence of failures of electrical components of the spacecraft with result. ing spuricas commands shutting down the reactor. An identical ground test unit, S1OFS-3,

opernied successfully for more than a year

before being shur down in 1966 . 


\section{EXPERIMENTAL POWER-REACTOR SYSTEMS}

\section{Space Propulaion}

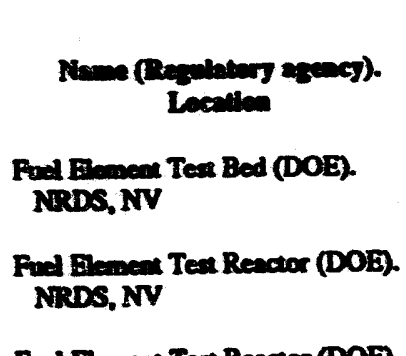

Fued Element Tex Reacor (DOE).

NRDS NV

Ground Experimental Engine

Experimea (DOS), NRDS, NV

\section{Goud Experimeatal Engine
Expecineat (DOS). NRDS, NV}

\& Nuclear Rocket Enpine Reactor Experimen (NERVA) (DOE) NRDS, NV

Nucten Rocker Engine Reactor Experiment (NERVA) (DOE) NRDS, NV

Nuclear Rocket Engine Renctor Experiment (NERVA) (DOE) NRDS, NV

Nuclear Rocket Engine Reactor Experiment (NERVA) (DOE) NRDS, NV

Nuclear Rocket Reactor Engine Symem Tear (NERVA) (DOE) NRDS, NV

\section{Nuclear Rocket Reactor}

Experimest (DOE). NRDS, NV

Naclear Rocket Reactor

Experinent (DOE). NRDS, NV

Necteer Rocteat Reactor

Experimeat (DOE). NRDS, NV
PARTI CMULAN REACTORS (DOMESTIC)

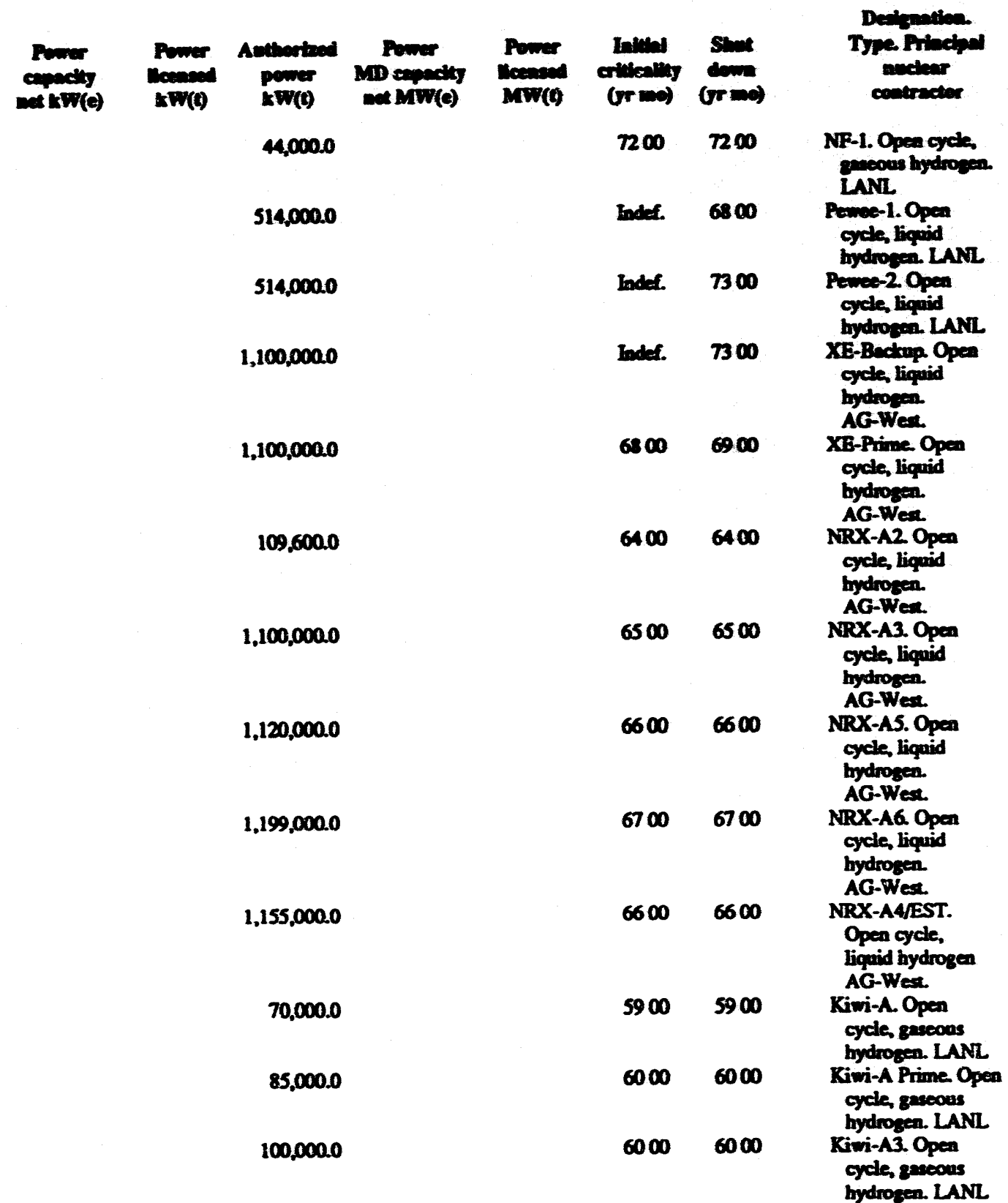




\section{Nuclear Rocket Reactor}

Experimen (DOE). NRDS, NV

boder Roctet Reactor

Experinem (DOE), NRDS, NV

Nuclear Rockea Reacuor

Experimed (DOE). NRDS, NV

Nuclear Rocket Reactor

Experimene (DOE). NRDS, NV

Nodeer Rocket Reactor

Experimen (DOE). NRDS, NV

Nuclear Rocket Reactor

Experimed (DOE). NRDS, NV

Nucber Rocket Reactor

Experimext (DOE). NRDS, NV

Nuclem Rockee Reactor

Experimeat (DOE). NRDS, NV
300,0000

$900,000.0$

$500,000.0$

$1,000,0000$

$950,000.0$

$1,070,0000$

$1,400.000 .0$

$4,200,0000$

\section{TEST, RESEARCH, AND UNIVERSITY REACTORS}

\section{A. General Irradiation Test}

\section{Name (Regplatory agency). Lecuilon}

$61006100 \quad$ Kivi-B1A. Opea

0200 bydiogen. LANL

Kiwi-BiB. Open

cycle, liquid

hydrogen. LANL

Kiwi-BAA. Open

cycle liquid

bydropen. LANZ

Kiwi-BAD. Opea

cycle, liquid

hydrogen. LANL

Kiwi-B4E Open

cycle, liquid

cycle, biquid

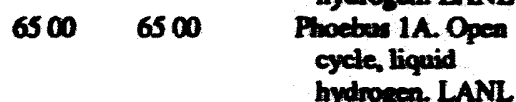

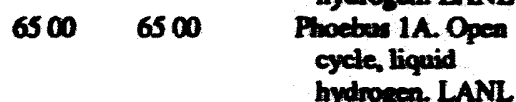

$67006700 \quad$ Phoetous 1B. Open

cycles liquid

bydrogen. LANL

68006800 Phoebus 2A. Open

cycle, liquid

bydrogen. LANL
Engiveering Tex Reactor (DOE)

INEL, ID

Feat Flex Teat Faciliy (DOE)

Henford Sice, WA

Gesenal Eloctric Texing Reactor

(NRC) Plessemion, CA

Miterials Texting Reacaor (DOE)

NEL, ID

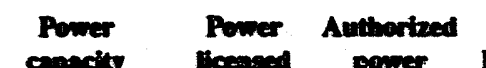

$175,000.0$

Simducty, OH

Weximghoose Texting Reacto

(NRC). Walz Mill, PA power

Power $\mathrm{MB}$ capacits
$\operatorname{met} \mathrm{MW}(\mathrm{S})$

Poner.

MW(t)

Initial

criticality

(rr mos)

5700

8100

$8002 \quad 9312$

$291,000.0$

$50,000.0$

$40,000.0$

58007700

$5200 \quad 7000$

$60,000.0$

$60,000.0$
$6100 \quad 7400$

$5900 \quad 6200$
Decigantiton.

Trpe Principed

enclear

centractor

ETR. Tank KE-GE

FFTF. Sodium cooled loop. WHC

GETR. Tank. Owmer.

MTR Tank

ORNL-ANL Blaw-Knox

NASA-TR. Trak.

NASA

WTR. Tank.
Reacior was shun down in 1973 for modificetions and insention of Sodiem Locp Srfery Facility (SLSF) loop. Operation resumed in 1975. Deacivased in 1981 and no bonger operable. The Fat Flax Tear Facility initined shuedowo activities $12 / 33$.

In August 1958 the MTR was opersed with m experimesed phucaium core at power levels up to $30,000 \mathrm{~kW}(0)$. I demanstruted the abiliny of plveonium fuel clements to perform setisfactority in a high-flux resenrch or test reactor. Opernion as a vea renctor was terminned 63069, and a Plutonium-240 (Pthoenix) cone was nn in FY 1970. Reactor was decommissicaed in 1974. 


\section{B. High-Power Research and Test}

\begin{tabular}{|c|c|c|}
\hline $\begin{array}{c}\text { Name (Regablory ageacy). } \\
\text { Lecatica }\end{array}$ & $\begin{array}{l}\text { Power } \\
\text { capacity } \\
\text { wet LW(e) }\end{array}$ & $\begin{array}{l}\text { Power } \\
\text { Bcemesd } \\
k W(9)\end{array}$ \\
\hline 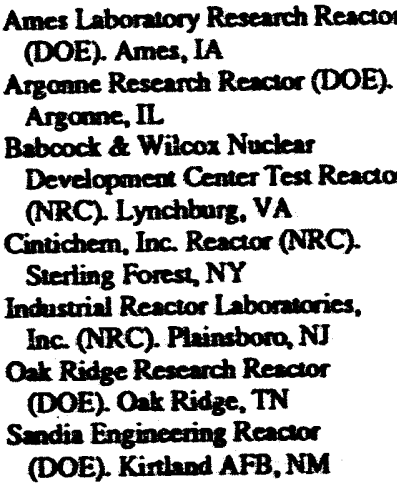 & & \\
\hline
\end{tabular}

\begin{tabular}{|c|c|c|c|c|c|c|}
\hline $\begin{array}{l}\text { Autharized } \\
\text { power } \\
\text { kW(t) }\end{array}$ & $\begin{array}{l}\text { Pomer } \\
\text { MD capseits } \\
\text { ed MW(e) }\end{array}$ & $\begin{array}{l}\text { Powes: } \\
\text { moenand } \\
\text { MW(1) }\end{array}$ & 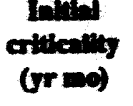 & (rimes) & 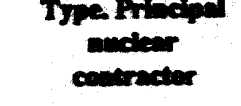 & \\
\hline $5,000.0$ & & & 6500 & 7700 & $\begin{array}{c}\text { ALRR. Heavy } \\
\text { war. AMF }\end{array}$ & \\
\hline $5,000.0$ & & & 5400 & 7900 & $\begin{array}{l}\text { CP.S. Heayy. } \\
\text { mer ANL }\end{array}$ & \\
\hline $6,000.0$ & & & 6400 & 7100 & $\underset{\text { Owiwh }}{\text { BAw Fool }}$ & \\
\hline $5,000.0$ & & & 6100 & 9000 & $\begin{array}{l}\text { CMR. Pool. } \\
\text { AMF }\end{array}$ & Beine decommistioned. \\
\hline $5,000.0$ & & & 5800 & 7500 & $\begin{array}{l}\text { PR: Pool } \\
\text { AMF }\end{array}$ & \\
\hline $30,000.0$ & & & 5800 & 8700 & ODR. TmL ORNL & Shen down-Detueted \\
\hline $5,000.0$ & & & 6100 & 7000 & at. Sundia & \\
\hline
\end{tabular}

King Intense Neviron Generator

(DOE). Los Alumos, NM

Kiwi-Transient Test Reacior

(DOE). NRDS, NV

Loss of Fluid Tess (DOE).

$55,000.0$

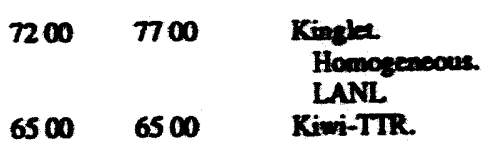

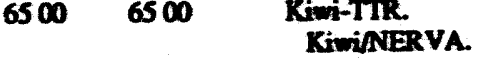

LANL

INEL, ID

$7800 \quad 8507$

OFT. Preseurized weer. EGEG-ID

Power-Burst Facility (DOE).

$$
\text { NEL. ID }
$$

$28,000.0$

SNAP-10A Transient Tea No. 2

(DOE). INEL, ID

\begin{tabular}{|c|c|c|}
\hline 7300 & 9200 & PBF. Open tank \\
\hline 6500 & 6601 & $\begin{array}{l}\text { SNAPTKAN-2 } \\
\text { Be-reflected } \\
\text { SNAP-10A. } \\
\text { AI-PRC }\end{array}$ \\
\hline
\end{tabular}




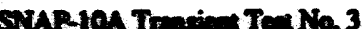
BOA REL, ID

Speciol Pomer Broumica Rouctor Tea Na 1 (DOS) EFL, ID Syciel Power Esconcion Rencto TE Na 2 (BOa) REL ID

\section{Spacid Powner Eremeion Reactor}

Ten Na 3 (DOE) DEL. ID

Speciel Power Excurcion Reacto

Tea Na 4 (DOE) NVI, ID

\section{Compral Research}

Acoulener Pulaed Fer Critical

Anerican Sunded be (NRC)

Momenia View, CA

Anumbr Cose Pubed Reactor

(DOD), Kialund AFB, Ear, NM

Argane CR-3, nebrin :

CP-3- (DOE). Paloe Pack, II.

Arpone Low Power Reseanch

Reactor (DOE), Argosese, II

Argone Nivional Leboseary

(DOE) Argones II

Argonne Nuclem Avenathy

for University Triming

(DOE) Argenne, II

Argonne Thennal Source

Reactor (DOE) Argones, II.

Aromics Intemution

(NRC). Cenoga Patt, CA

Baboock \& Wilcon Lymctibung

Pool Reactor (NRC)

Lymctbure. VA

Batelle Memorial Instiune

(NRC). Weat Jefferson, OH

Bialogical Reseach

Reactor (DOE). Areares, II

Broolchaven Gon hine Resengch

Reactor (DOE). Uptan, NY

3000

250.0

$1,000.0$

$2,000.0$

200.0

$20,000.0$

\begin{tabular}{|c|c|}
\hline 6409 & 600 \\
\hline 5500 & 600 \\
\hline 6000 & 600 \\
\hline 5800 & 600 \\
\hline 6200 & 7000 \\
\hline
\end{tabular}

67007300 APFA-II. Fax

aA

5809 coes UTR-1.

\section{Grit.}

Gophiedwenter

6700 ACR. U-Z

bydride. GA

44006309

cese.

water. Met Lab.

62007000

Jugzemane.

Gophinefwater.

ANL

AGN-201-108.

Howneg-solid. AGN

$5700 \quad 7200$

$5700 \quad 7200$

Argonaue (CP-11)

Anl.

$5700 \quad 8800$

ATSR. Thermal ANR

$5700 \quad 5800$

$5800 \quad 8100$

L-47. Homogeneons.

AI

LRP. Pool Owrer

$56007400 \quad$ BRR. Pool. AMF

64009200

JANUS. Tant. ANL

50006900 BCRR. Aircooled.
The APFA-III wa provioundy operned as the kuxl A Prompt Critical Aveonbly at

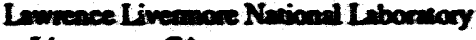

- Liveduarar CA.

This reactor wes shipped sbmond for exthibition

puposes in the USAEC Anome for Peace

Exhibit in the Tokgo Imeanetional Trade Fair in 1959, and in Ciso, Egrp, and Labore. Pariation, in 1960.

In 1977 the Amavir Core Pulsed Reactor

(ACPR) was shat down. Afier replacenent of

fvel and other modifications, the unit was

rensened the Asmular Core Resench Resceor.

After the assembly and operation of this reactor in the government exhibit at Geneva in 9/58, it was dismmated and rewmed to ANL, where it was rebuilt as a $250 \mathrm{~kW}(\mathrm{l})$ Ingapernouse. 


\section{General Research (Continued)}

\section{Name (Regabery areacy)
Locetion}

Brookhnven Nentron Source

Reactor No. 1 (DOE).

Upton, NY

Broolthavea Neutren Source

Reaction Na 2 (DOE).

Upton, NY

Bulk Stielding Reactor (DOE).

Ouk Ridge, TN

Chicago Pile 1, rebuilt as

CP-2 (DOE), Chicaga, II

Cuniss-Wrighn Nuctear Research Laborony of the Common-

wealth of Peansylvanin (NRC)

Quehwasa, PA

L DOE Demonstration Reactor

(DOE) Oak Ridge, TI

\section{Europena-Asinn Exhibit Program} (DOE). Oak Ridge, TN

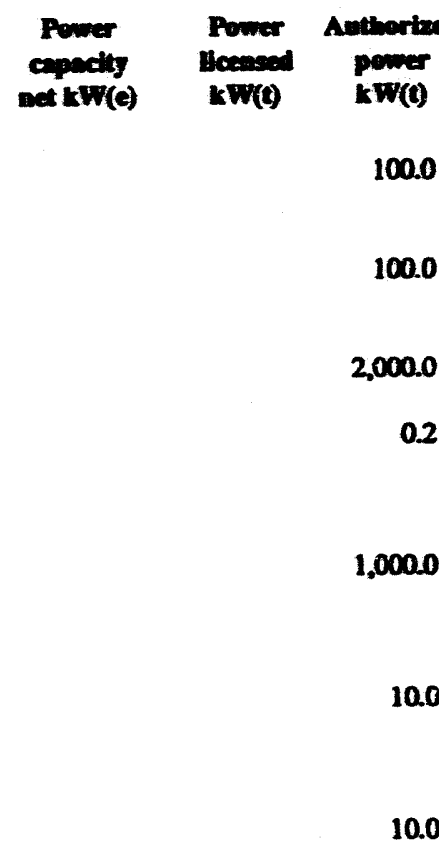

Fast Neutron Source Reactor (DOE). Upton, NY

Fast Source Reacior (DOE).

INEL Site, ID

General Atomics Technologies

(DOE). San Diego, CA

Health Physics Research Reacto (DOE). Oak Ridge, TN

High Texpermure Larice Test

Resctor (DOE). Richland, WA

\section{Deringation. \\ Trine Principal \\ Emcles: \\ conturecter}

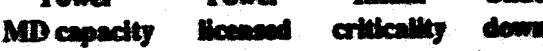

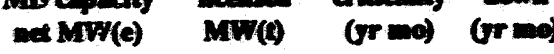

ScHizo. Tat AU.

lne.

PHRENic Tak AU,

lac.

$5000 \quad 9100$

BSR. Pool Oanl

Shat down-to be defueled.

$4200 \quad 5400$

CP-2. Graphite.

Nethe

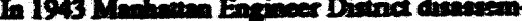 bled Chiceno Pils 1 and rebeile a Palos Pats.

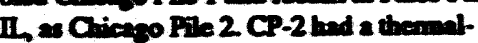

$5800 \quad 6600$

CWRR. Pool Omser
Demo Reac Pool
Lockheed

6300

6900

EAEP. Pool

Lockhoed

$\begin{array}{lll}6700 & 7000 & \begin{array}{c}\text { BNLAFS-1. Fast. } \\ \text { BNL }\end{array} \\ 5900 & 9300 & \\ 5000 & 6000 & \begin{array}{c}\text { TRIGA-Mk II. U-Z } \\ \text { bydride Owmer }\end{array}\end{array}$

$6200 \quad 9100$

$6700 \quad 7100$ bydride Owner

HPRR. Fast burst

ORNL

HTLR Gaphive
This rector was forwexty celled the Lutin American Desoncetration Reactor and was operated initielly in Sio Prubo, Bnail, 1069. his currem ty in storage at Onk Ridge. This reactor was cperated in the USAEC

Aloms for Peace Exhibin in Vienan, Austria, 6163; Belgnede, Yuzodinvis 9653. Modrid, Spein, 464; Libeon,

Porugel, 465; Urecte, Nethertand, 3/66; Dublin, Iretend, 966 to 10665, Antenra. Turkey, 4/67 10 5/67; Tehren, Iren, 11/67 wo 12/67; Tripei. Triwen, 4/68 20 5/68; Secoul,

Korea, 9/68 10 10688; Manith, Philippines,

2/69 vo 3/69; and Buchareas, Romania, 10/69.

This TRIGA-Mk II was operned at the New Dethi Wortd Agricultural Fair in 1960.

It was diemmited for storne in Califomia

by Chevron USA Corporation. Ommer.

Wodd Arriculumal Fair-U.S. Exhibit

Reactor.

Shun down-Defueled. 
Chicaso, II

Kinctic Experiment on Wher Balers (ARC) Suma Smema $C A$

Livemone Pool Type Reactor (DOE) Livermone, CA

Livensone Wmer Boiler

(DOE). Livermose, CA

Lockheed Aincrafi Corp. (NRC)

Desusonville GA

Los Alamos Fast Reactor

(DOE). Los Alemos, NM

Los Almos LOPO Reacior

(DOE) Los Almos, NM

Los Alamos Waver Boiler

(DOE). Los Alamos, NM

Los Almos Water Boiler

(DOE) La Alemos, NM

Lovisimn Sime University

Nuctem Science Center

w

$3,000.0$

0.5
(DOE), Onk Ridge, TN

Low Tempersure Neutron

Irrediacion Facility (DOE).

Onk Ridge, TN

NASA Mock-Up Reactor

(NRC) Sundualy, OH

Neutron Radiography Facility

(DOE). Hanfond Sive, WA

Northrop Corpornte Libontories (NRC) Hawthome, CA

Nuclear Effects Reactor (DOE). NTS, NV

250.0

100.0
Nechen Effects Reveror (DOE)

Sm Diezo, CA
5600

6700

5600

6700

$5700 \quad 8000$

$5300 \quad 6100$

60006000

$4600 \quad 5300$

4400440

44005000

$5000 \quad 7400$

$6500 \quad 6600$

ARR (L-SA)

Homogeneene:

AI

KEwB.

Homogenecue. A

UPR Tak FW

LWB. Homogeneows.

AI

No Dese. Pool.

Lockheed

Clemecatine Fext

plutoniven fuel.

mencury cooled.

LANL

LOPO. Howngenecons.

LAMI

HYPO. Howosenecus

LAN

SUPO. Homagenoour.

LANL

SNARE Pool.

Sendia

$\begin{array}{ccc}5000 & 6800 & \begin{array}{c}\text { ITR. Tank } \\ \text { ORNL } \\ \text { LTNIF. Pool. } \\ \text { ORNL. }\end{array} \\ 8600 & 9100 & \begin{array}{c}\text { MUR. LWR. } \\ \text { Lockhoed }\end{array} \\ 7700 & 9300 & \begin{array}{c}\text { NRF. U.Z } \\ \text { hydride WHC }\end{array} \\ 6300 & 8600 & \begin{array}{c}\text { TRIGA-MK F. U-Z } \\ \text { hydride. GA }\end{array} \\ 6200 & 7000 & \begin{array}{c}\text { FRAN. Prompt burs. } \\ \text { LLNLPPC }\end{array}\end{array}$

5900

6400
Ommer: Amoour Revench Foundmion.

The KEWB reweor was cpereled by AI from

1956 10 1967 a the Kinetic Expeximent on

Weer Bciles. Omner. Rock well Inesmotionel

No power lioded tumiene
In 1965 and 1966 this reactor operated at Sindia, NM, as SNARE. Prior to that time it was operned a DNEL as the Shield Tea Pool Reactar (SUSIE) in the Aircnif Nuclear Propulsion Progenen from 1959 wo 1962. was sture down in 1966 and trenderred 10 Louisiana Sime University 6166, where in was never assembled. Owner. Phillips Petroleum Ca.

The Neusron Radicezraphy Facility was ured to perform neutron radiography of reactor fued pins. The reactor was shue down in 1989 and has been convented to a fuel storage basin. Owner. Space Rediation Laborstory. This TRIGA reactor was capable of being polsed and of steady-sate operation.

Unil mid-1967 FRAN was operted by

LUNL at the Nevada Ter Site, and until 1970 it was operated in the former ML-1 reactor area i INEL In mid-1970 it was transferred back to LLNL. Fuel was removed for processing. 


\section{TEST, RESEARCH, AMD UNMERSTY REACTORS}

\section{General Research (Continued)}

\section{Name (Regulatory agency). Lecotion} (NRC). Santa Susana, CA

Onk Ridze Graphite Reactor (DOE), Oak Ridge, TN

Pawling Research Reacto

(NRC) Pawling, NY

Prysical Constants Tere

Rediation Effocts Reactor

(NRC). Dawsonville, GA

$\ddot{\alpha}$
Nuclear Examination Rexctor

Reactor (DOE), Richland, VA

\section{PART I CNLLW PEACTORS (BomESTC)}

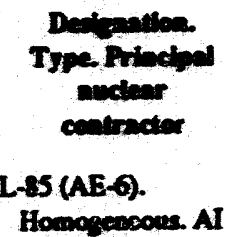

43006300

$5800 \quad 7100$

$5500 \quad 7200$

$5800 \quad 7000$
3.500 .0

3,0000
Rockwell Intemational (NRC)

Canoga Part, CA

Sendia Pulsed Reactor

(DOE). Kiniand AFB, Easa, NM

Shield Test and Irradiation

Reactor (DOE). Santa

Susana, CA

Thermal Test Reactor No. 2

(DOE). Richland, WA

Tonrey Pines, TRIGA-Mk III

Reactor (NRC). La Jolla, CA

Tower Shielding Reactor

(DOE), Oak Ridge. TN

Tower Shiekting Reactior

No. II (DOE). Oak Ridge, IN

UTR Teat Reactor (NRC).

Mountaia View, CA
1.000 .0
$5800 \quad 7400$

$6100 \quad 6700$

61007200

$5500 \quad 7200$

66007300

$5400 \quad 5800$

$6000 \quad 9200$

$6100 \quad 6300$

$7200 \quad 800$
L77. Homoneneous.

AI

SPR. Prompe burs

Sendiv.

STIR. Pool A

PNL

TRGA-MR II U-Z

mydride. Owner

TSR. BSR-type in

unk ORNL

TSR-2. Ligh

waver. ORNL

No Dere.

Graphivetwater.

Omer.

WNTR Talk Weat
TTR-2. Grophine.
Ownenchip of this recetor was unoferved

co Nonth Amerion Rockwell 12/1 and was

redeximned the Nudeer Exemination

Reactor or L-S wher inm AE-6. The AE-6, iloo decipmed WBNS, wa biil and fin cposued a Doney, CA. F was moved to

Smasuran in 195.

Omber Unied Nuclear Cop.

The RER was previounly wed in the tesminened Airenaft Noclear Propulsion

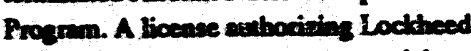
to coperte the reactor at a commencial facility was iswed 7/62, and 8462 the USAF tnom fened be faciling so the Geneenl Senvices Adminimution. Lockhoed scepuired the sitle so the facility $3 \times 65$.

This reaceor was previondy decignated STF

Owner. Generel Alomic

Shat down-To be defieled.

Owner: American Rediver \& Sundand Smitry Copp for SAMP Shiedd Tea Fenitiy. 


\section{E. Univerelty Research and Teaching}

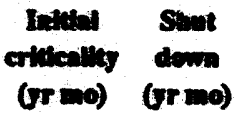

power

Mitennests

an MWr(e)
Pume MW(0) (rr mo) (or mo)

\section{Name (Regablabery revect) Locetite \\ ORC) Universe \\ Celifomia Polyrechnic \\ State University (NRC) \\ Sen Luis Obispo, CA}

Power
capecily
nativite

Powner
licenend
kW(O)

6700

Catifomin, Berteley,

Univeruity of (NRC)

Berteley, CA

Califomia, Los Angeles,

University of, School of

Engineering and Applied

Science (NRC). Los Angeles,

CA

California, Santa Barbare,

University of (NRC). Santa

Barbarn, CA

Catholic University of

America (NRC)

Weshington, DC

Colondo State Univerzity

(NRC). Fon Collins, $\mathrm{CO}$

Columbia University (NRC).

New York, NY

Delaware, University of

(NRC). Newark, DE

Georgis Institute of

Technology (NRC)

Adlenta, GA
$1,000.0$

100.0

6600

8712

TRIGA-Mk III.
U-Zrhydride.

GA

60008400 Eductor.

Gophite/water.

AMF

$7400 \quad 8600 \quad$ L-77.

Homogeneous. AI

$5700 \quad 8600 \quad$ AGN-201-101.

Homog. solid.

AGN

$5700 \quad 7400 \quad$ AGN-201-109.

Homog, solid. AGN

Licensed 8500

TRIGA-Mk II.

U-Zrhydride.

GA

\begin{tabular}{|c|c|c|}
\hline 5800 & 7800 & $\begin{array}{l}\text { AGN-201-113. } \\
\text { Homog. solid. } \\
\text { AGN }\end{array}$ \\
\hline 6800 & 8500 & $\begin{array}{l}\text { AGN-201-104. } \\
\text { Homog. solid. } \\
\text { AGN }\end{array}$ \\
\hline
\end{tabular}

The Columbia University TRIGA-Mk II was licensed to operate by NRC. However, the City of New York has not authorized operation. Therefore Columbia Universiry has not procured fuel. The license was teminuted in 1985.

AGN-201-104 operated at the University of Akron (Ohio) from 1957 until transferred to the Georgia Institute of Technology in 1967. Operations at that facility began in 1968. Decommissioning of AGN-201-104 was achieved in 1986. 


\section{TEST, RESEARCH, AND UNVERSTY REACTORS}

\section{E. University Recearch and Teaching (Continued)}

$$
\text { Nome (Reypladery eswes) }
$$

Kmane, Univerity of

ARRC), Lawsence, KS

Lelaad Stenford Univerim

(NRC). Pub Aro, CA.

Memphis Sucs Univessing

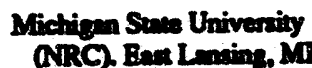

Mieciecipipi Sums Universing

(NRC). Sume Colleger ins

Nevade, Univerniny of (NRC).

Rean, NV

North Carolina Stave

University (NRC)

Ruleigh, NC

Otinhome, University of

(NRC). Normm, OK

Oregon State University

(NRC). Corvalitis, OR

Polynectnic Instimue of New

Yotk (NRC). New Yort, NY

\section{Pueno Rico Nuclent Cenver} DOE). Mayagiea, $\mathrm{FR}$

Pueno Rico Noclear Cender (DOE). Mayagien, PR

250.0

2,0000
PART I CIVILIAN REACTORS (DOMESTIC)

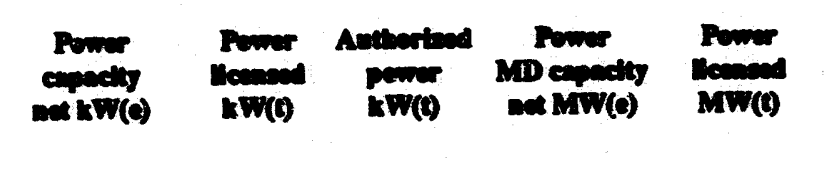

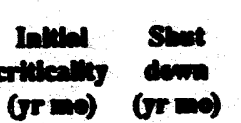

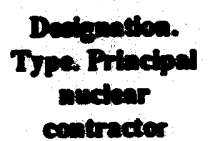

61008700

100

$5900 \quad 7400$

$7700 \quad 8500$

69008900

Model 4180.

Poot BAC

No Deser. Pool.

GE

AGN-201-108.

Hownes solid.

AGN
RUGA-MKI.
U-Zx hydride

GA

RRR.

Homogeneous.
Owner. NSA

A

$\begin{array}{lll}6300 & 7400 & \text { L-77. } \\ 6000 & 7300 & \text { Homogeneons. AI } \\ \text { No Desg. } \\ \text { Grophive/waser. }\end{array}$

$58008800 \quad \begin{aligned} & \text { Cook } \\ & \text { AGN-211-102 } \\ & \text { Homog. solid, }\end{aligned}$ pool. AGN

$5800 \quad 7400 \quad$ AGN-201-114.

Homos solid.$$
\text { AGN }
$$

$67007400 \quad$ AGN-201M-105.

Homos. solid.
AGN

$5900 \quad 7900 \quad$ L-77.

\begin{tabular}{|c|c|c|}
\hline 7200 & 7600 & $\begin{array}{l}\text { TRIGA-FLIP. } \\
\text { Pool-TRIGA } \\
\text { core GA }\end{array}$ \\
\hline
\end{tabular}

Homoneneous. AI
In 1957-1962, AGN-201M-105 was owned and operated by the National Naval Medical Center, Bethesda, MD. Tile to the reactor was transferred to New York University early in 1964. A license to opernite was issued 467. Owner. The Center for Envirommental and Enerny Research (formerty Pueno Rico Nuclear Center).

This reactor was operated at the Pueno Rico

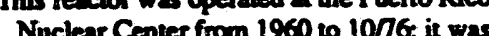
Nuclear Cencer from 1960 to 1076, il was converted to TRIGA-FLIP in 1972. It has been moved to the Neutron Radiography Facility a
the National Engineering Labortiony in Idaho. 
Texns at Austin, University of (NRC2. Austin, TX

6300

9100

Tuakegee, AL

Utha, University of (NRC)

Selt Leke City, UT

Virginin, University of (NRC).

Charlotesville, VA

Vinginia Polyrechnic Institute

100.0

Weshington, University of (NRC)

100.0

Sesule, WA

Wea Vinginin Universiry (NRC) Morgantown, WV

Willien Marsh Rice University

(NRC). Houston, TX

Wyoming, University of (NRC)

Lanmie, WY
Tuskesee Instime (NRC)

(NRC). Blacksbure. VA

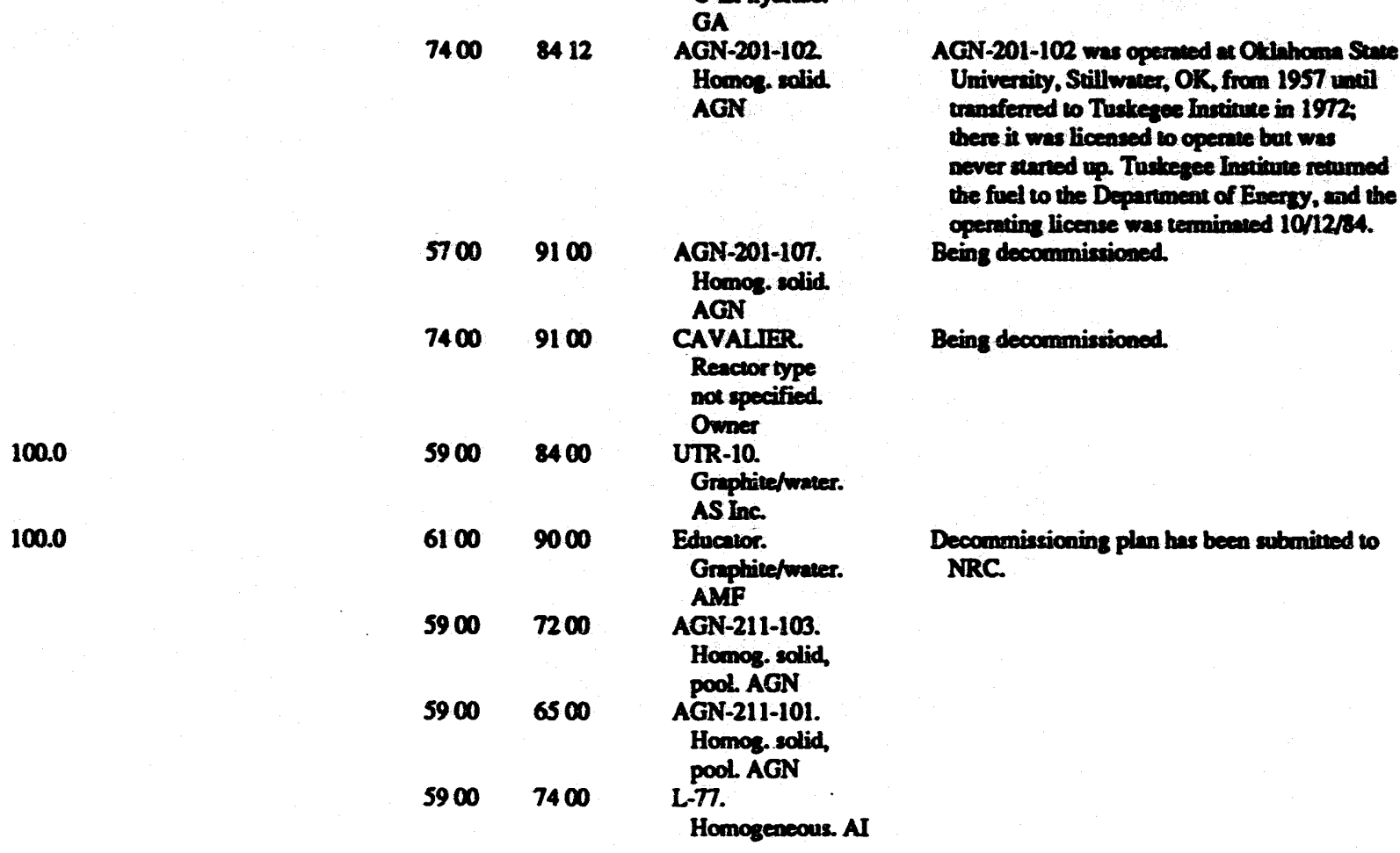

1. MATERIALS PRODUCTION

\begin{tabular}{|c|c|c|c|}
\hline Name (all owned by DOD) & Dealizantion & Loention & $\begin{array}{l}\text { Nudiear } \\
\text { destger }\end{array}$ \\
\hline $\begin{array}{l}\text { B Reactor } \\
\text { C Reactor } \\
\text { C Reactor } \\
\text { D Reactor } \\
\text { DR Reactor } \\
\text { F Reactor } \\
\text { H Reactior } \\
\text { KE Reactor } \\
\text { KW Reactor } \\
\text { L Reactor } \\
\text { P Reactor } \\
\text { R Reactor }\end{array}$ & $\begin{array}{l}\text { B Reactor } \\
\text { C Reactor } \\
\text { C Reactor } \\
\text { D Reactor } \\
\text { DR Reactor } \\
\text { F Reactor } \\
\text { H Reacior } \\
\text { KE Reactor } \\
\text { KW Reactor } \\
\text { L Reactor } \\
\text { P Reactor } \\
\text { R Reactor }\end{array}$ & $\begin{array}{l}\text { Richland, WA } \\
\text { Richlend, WA } \\
\text { Aiken, SC } \\
\text { Richland, WA } \\
\text { Richland, WA } \\
\text { Richland, WA } \\
\text { Richland, WA } \\
\text { Richlend, WA } \\
\text { Richlend, WA } \\
\text { Aiken, SC } \\
\text { Aiken, SC } \\
\text { Sivannah River Pinnt, }\end{array}$ & $\begin{array}{l}\text { Du Poat } \\
\text { GE } \\
\text { Du Pont } \\
\text { Du Pont } \\
\text { GE } \\
\text { De Pont } \\
\text { GE } \\
\text { GE } \\
\text { GE } \\
\text { Du Pont } \\
\text { Du Pont } \\
\text { Du Pont }\end{array}$ \\
\hline
\end{tabular}

\section{PART II PRODUCTION REACTORS}

Shet

(Tr mo) Commeat 


\section{PROCESS DEVELOPMENT}

\begin{tabular}{|c|c|c|c|c|c|c|c|c|}
\hline Name (all owned by DOD) & Designation & Location & $\begin{array}{l}\text { Nediear } \\
\text { dedigner }\end{array}$ & Type & $\begin{array}{l}\text { Anthorbed } \\
\text { power } \\
\text { kW(i) }\end{array}$ & $\begin{array}{l}\text { Imitial } \\
\text { criticallity } \\
\text { (yr mos) }\end{array}$ & $\begin{array}{l}\text { Shint } \\
\text { down } \\
\text { (jo wos) }\end{array}$ & Conament \\
\hline $\begin{array}{l}\text { Henford } 305 \text { Test Reactor } \\
\text { Letice Test Resetor }\end{array}$ & $\begin{array}{l}\text { HTR } \\
\text { LTR }\end{array}$ & $\begin{array}{l}\text { Richland, WA } \\
\text { Savanmah River Plant, } \\
\text { Aiken, SC }\end{array}$ & $\begin{array}{l}\text { Du Pont } \\
\text { Du Poot }\end{array}$ & $\begin{array}{l}\text { Graphive } \\
\text { Heavy water }\end{array}$ & 1.0 & $\begin{array}{l}4400 \\
6700\end{array}$ & $\begin{array}{l}7600 \\
7900\end{array}$ & Negligible power. \\
\hline Process Development Pile & PDP & $\begin{array}{l}\text { Savarnah River Plant, } \\
\text { Aiken, SC }\end{array}$ & Da Pom & Heavy water & 1.0 & 5300 & 7900 & \\
\hline SR 305-M Test Pile & Test Pile & $\begin{array}{l}\text { Savannah River Plunt, } \\
\text { Aiken, SC }\end{array}$ & Du Pont & Graphite & 1.0 & 5300 & 8300 & 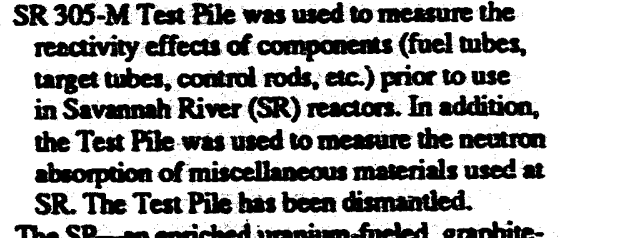 \\
\hline $\begin{array}{l}\text { Standard Pile/ Subcritical } \\
\text { Experimentul Complex }\end{array}$ & SPSE & $\begin{array}{l}\text { Savamah River } \\
\text { Labontory, Aiken, SC }\end{array}$ & Du Pont & Graphite & 2.0 & 5300 & 7900 & 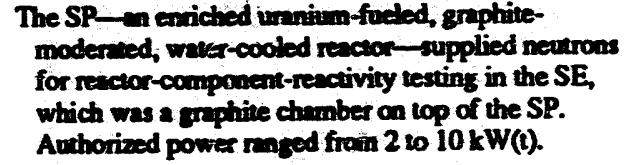 \\
\hline
\end{tabular}

\section{DEFENSE POWER-REACTOR APPLICATIONS}

\section{A. Remote Installations}

Reactors in the Amny Power Program are designated to reflect mobility chancteristics, power range, development sequence, and field sequence. The first capital letter indicates mobility characteristics: $S$ (stutionary operation), not designed for subsequent relocation; P (portuble), semimobile, stationary operation, capable of being dismantled and reassc. abled for use in successive locations; and M (mobile), capable of being moved intace, or virtually intact, for use in successive locations. The second copital letter

\section{Name (all owned by DOE)} Location

Porrable Medium Power Plant No. 1. Sundance, WY

Portable Medium Power Plant, No. 2A. Camp Century. Greentand

\begin{tabular}{|c|c|c|c|c|c|}
\hline Designation & $\begin{array}{c}\text { Power } \\
\text { capacity } \\
\text { net } k W(e)\end{array}$ & $\begin{array}{l}\text { Authorized } \\
\text { power } \\
k W(t)\end{array}$ & $\begin{array}{l}\text { Initial } \\
\text { criticallity } \\
\text { (yr mo) }\end{array}$ & $\begin{array}{l}\text { Shat } \\
\text { dowsat } \\
\text { (pr mo) }\end{array}$ & $\begin{array}{l}\text { Princtpal } \\
\text { nuclear } \\
\text { contractor. } \\
\text { Reactor type }\end{array}$ \\
\hline PM-1 & $1,000.0$ & 9.370 .0 & 6200 & 6800 & $\begin{array}{l}\text { Martin. } \\
\text { Pressurized water. }\end{array}$ \\
\hline PM-2A & 1.560 .0 & $10,000.0$ & 6000 & 6300 & $\begin{array}{l}\text { Alco. } \\
\text { Psessurized water. }\end{array}$ \\
\hline
\end{tabular}

\section{PART III MILITARY REACTORS}

incicates the power range as measured by design capacity for contimucus opernion: $L$ (low) 100 to $1000 \mathrm{~kW}$ (e) M (medium), 1000 to 10,000 kW(e); and H (high), 10,000 kW(e) or more. Arabic numerals indicate order in which phants having the same mobility and power chancteristics are initiated. If not followed by an additions letter, the desimation indicates a prototype or pilat plant. The last capial leter (when present) indicates the alphabetical onder in which field plants of a specific type are initiated.

The PM-2A was shot down 7/9/63 and dismentled during 4/64 to 6/64. The reactor vessel was then used by INEL for NDT (nil ductility trensition temperenure) investigevions of menerinls thas had been subjected to long-term ions of mmerials that had been subjected to longterm

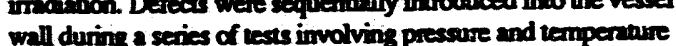
conditions which exceeded the range permined in operating conditions which exceeded the range permined in operniting nuclear power plans: The finil ten on 11/18/66 resulted in a which had been previousty prodicted to ceuse frilure. The test program confirmed labortiony data on the adequacy of reactor-opereting linitations to prevent britle fracture of a pressure vessel 


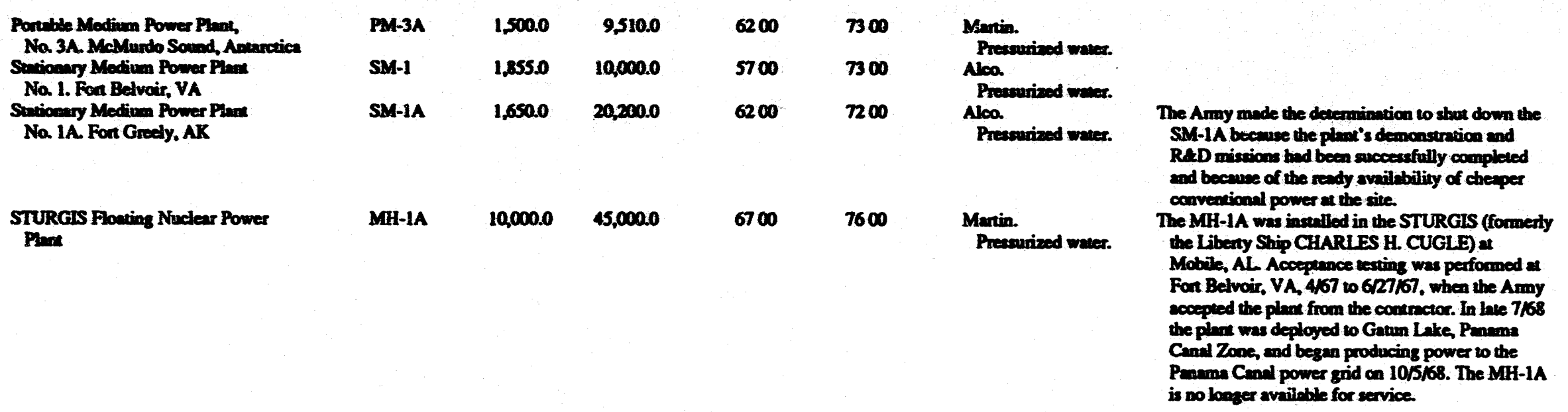

\section{B. Propulsion (Naval)}

SSN, Submarine (Nuclear Propulaion).

SSBN, Flea Ballixic Missile Submarine (Nudear Propulsion).

CON, Guided Miasle Cruiser (Nuclear Proputrion).

Name (cll owned by US. Navy)

- NAUTILUS

SEAWOLF PWR

SEAWOLF Sodium Reacto

\section{SKATE}

SWORDFSH

SARGO

SEADRAGON

SKIPJACK*

TRITON (2 Reactors)

HALIBUT

SCAMP*

SCORPION

SCULPDN

SHARR*

SNOOK

THRESHER

PERMTT

PLUNGER*

BARB*

TULLIBEE

GEORGE WASHINGTON *

PATRICK HENRY *

THEODORE ROOSEVELT*

ROBERT E LEE*

ABRAHAM LINCOLN*

POLLACK*

HADDO

JACK*

\section{Deslgantion}

Sunpuniler

SSN571

SSN575

SSN575

Electric Boat (Grotion)

Electric Bont (Groton)

Eloctric Boat (Groton)

Electric Bont (Growon)

Ponsenouth

Mare Island

Portsmouth

Electric Bost (Groton)

Blectric Bost (Groton)

Mare Ishond

Mare Island

Electric Bont (Groton)

Ingalls

Newport News

Ingalls

Ponsmounh

Mare Isind

Mare Islend

Ingalls

Electric Boat (Groton)

Elextric Bon (Groton)

Electric Boent (Groton)

Mare Isiand

Newpon News

Electric Bost (Groton)

NYSC

NYSC

Ponthsmouth
Soms.

5400

6000

6000
5700

5700

5800

5800

5900

5800

5900

5900

6100

6000

6100

6000

6100

6100

6200

6200

6300

6000

5900

6000

6000

6000

6000

6300

6400

6500

Sweo
dow0
8000
8600
5900
8500
8700
8600
8300
8900
6800
7600
8700
6800
8900
8900
8600
6300
9000
8900
8900
8700
8400
8300
8100
8300
8100
8800
9000
8900

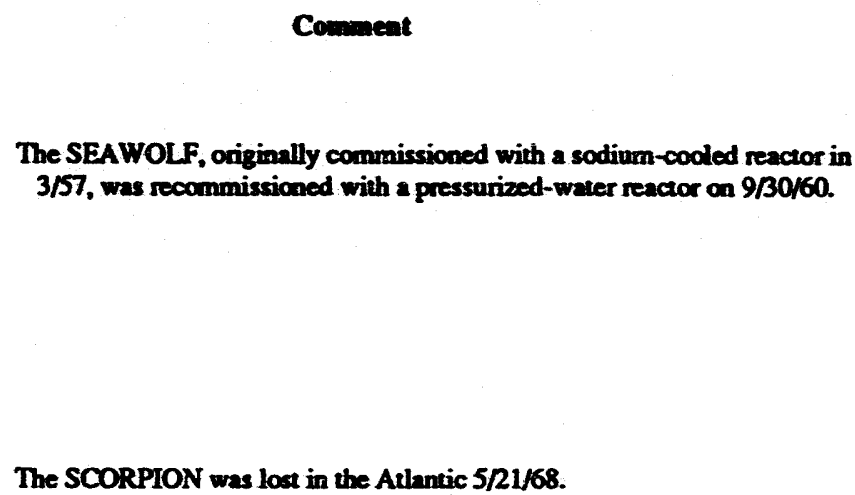

Commeneat

The SEAWOLF, originally commissioned with a sodium-cooled reactor in 3/57, was recommissioned with a pressurized-water reactor on 9/30/60.

The SCORPION was lost in the Atlantic 5/21/68.

The THRESHER was lost in the Allantic 4/10/63. 


\section{DEFENSE POWER-REACTOR APPLICATIONS}

\section{B. Propulaten (Naval) (Continued)}

Name (all nomed by US. Nang)

\section{TrNosA*}

DACE*

ETHAN ALLEN

SAM HOUSTON:

THOMAS A. EDSON

JOHN MARSHALL

GUARDFAH:

FLASHER:

GREENING

LAFAYETTE*

ALEXANDER HAMIILTON*

THOMAS JEFFERSON

ANDREW IACKSON

JOHN ADAMS

HADDOCR

JAMES MONROE*

NATHAN FALE:

WOODROW WILSON

HENRY CLAY

* JAMES MADSON

TECUMSEH

DANIEL BOONE

JOHN C. CALHOUN

ULYSSES S GRANT"

VON STEUBEN

CASDMR PUIASKT

NATHANAEL GREENE

BENUAMIN FRANKLN

GEORGE BANCROFT

LEWIS AND CLARK

QUEENFISH*

RAY

GEORGE C MARSHALL*

HENRY L. STMMSON

GEORGE WASHINGTON

CARVER*

FRANCIS SCOTT KEY

WIL ROGERS

LAPON

SEA DEVIL

GUTTARRO*

GLENARD P. LIPSCOMB*

RICHARD B. RUSSELI

BATONROUGE

TEXAS (2 reactors)

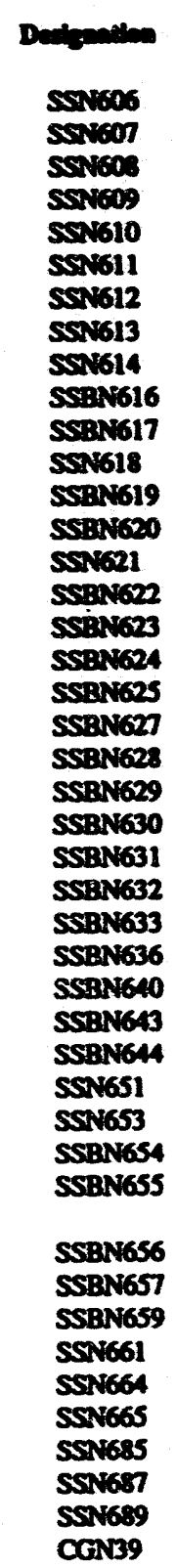

Sipponerere

Pendonowich

Inentin

Bectic Bom (Growa)

Nowpont New

Bectric Boen (Grom)

Newpen Nems

NYSC

Electric Boes (Growa)

GD (Quincy)

Electric Bom (Groson)

Electric Bon (Growon)

Newpont News

More Island

Porseno

Buglll

Newpon Newa

Blectric Boen (Growan)

Merelainad

Newpont News

Newpor News

Blectric Boen (Grocon)

Mrre Rlend

Newpon News

Electric Boet (Groton)

Nempor News

Electric Boen (Growon)

Portumoseh

Electric Boex (Groecon)

Electric Boes (Groton)

Nemport News

Newant News

Nempor News

Newpon News

Electric Boat (Growa)

Sut.

Comonent

$6600 \quad 9200$

- The defueled reactor comparimena has beea removed and pleced in a govemaneat burid grouad. 


\section{DEVELOPMENTAL POWER}

A. Electric-Power Experiments and Prototypes

\begin{tabular}{|c|c|c|c|c|c|c|}
\hline $\begin{array}{l}\text { Nane (owmer). } \\
\text { Location }\end{array}$ & Declanation & $\begin{array}{l}\text { Power } \\
\text { capacity } \\
\text { net \&W(e) }\end{array}$ & $\begin{array}{l}\text { Avthortied } \\
\text { power } \\
\text { kW(0) }\end{array}$ & $\begin{array}{l}\text { Intial } \\
\text { criticallity } \\
\text { (or mo) }\end{array}$ & $\begin{array}{l}\text { Shat } \\
\text { down } \\
\text { (ormo) }\end{array}$ & $\begin{array}{l}\text { Reactor type } \\
\text { Priactpol } \\
\text { nuclear } \\
\text { contractor }\end{array}$ \\
\hline $\begin{array}{l}\text { Gas Cocled Reactor Experiment } \\
\text { (DOE). INEL Site, ID }\end{array}$ & GCRE & & $2,200.0$ & 6000 & 6200 & $\begin{array}{l}\text { Gas cooled, light } \\
\text { water moderntod. } \\
\text { AGN }\end{array}$ \\
\hline $\begin{array}{l}\text { Mobile Low Power Plant No. } 1 \\
\text { (DOE). NEL Site, ID }\end{array}$ & ML-1 & 300.0 & $3,300.0$ & 6100 & 6500 & $\begin{array}{l}\text { Gas cocled, light } \\
\text { water moderated. } \\
\text { AGN }\end{array}$ \\
\hline $\begin{array}{l}\text { Sutationary Low Power Plant No. } 1 \\
\text { (DOE). NELL Site, ID }\end{array}$ & SL-1 & 300.0 & $2,200.0$ & 5800 & 6100 & Boiling water. ANL \\
\hline \multicolumn{7}{|c|}{ B. Propulsion Experiments and Prototypes } \\
\hline $\begin{array}{l}\text { Aircraft Reactor Experiment (DOE) } \\
\text { Ouk Ridge, TN }\end{array}$ & ARE & & $1,500.0$ & 5400 & 5400 & Molten salk ORNL \\
\hline $\begin{array}{l}\text { Experimerud Propulsion Test Resctor } \\
\text { (DOE). NTS, NV }\end{array}$ & TORY IIA & & 150,0000 & 6000 & 6100 & Air cooled. LLNL \\
\hline $\begin{array}{l}\text { Experimental Propulsion Test Reactor } \\
\text { (DOE) NTS, NV }\end{array}$ & TORY IC & & $600,000.0$ & 6400 & 6400 & Air cooled. LINL \\
\hline $\begin{array}{l}\text { Heat Tramefer Reactor Experiment } \\
\text { No. } 1 \text { (DOE). INEL Site, ID }\end{array}$ & HTRE-1 & & $20,000.0$ & 5600 & 5700 & Air cooled. ANPD \\
\hline $\begin{array}{l}\text { Heat Truafer Reactor Experiment } \\
\text { Na. } 2 \text { (DOE) INEL Site, ID }\end{array}$ & HTRE-2 & & $14,000.0$ & 5700 & 6100 & Air cooled. ANPD \\
\hline $\begin{array}{l}\text { Heat Trusfer Reactor Experiment } \\
\text { Na. } 3 \text { (DOE). NEL Site, ID }\end{array}$ & HTRE-3 & & $32,000.0$ & 5800 & 6100 & Air cooled. ANPD \\
\hline $\begin{array}{l}\text { Sinall Submarine Reactor } \\
\text { Prototype (DOE), Windsor, CT }\end{array}$ & Sic & & & 5900 & 9300 & $\begin{array}{l}\text { Pressurized water. } \\
\text { GE }\end{array}$ \\
\hline $\begin{array}{l}\text { Submarime Advanced Reactor } \\
\text { Protoxype (DOE). West Milion, NY }\end{array}$ & S3G & & & 5800 & 9100 & $\begin{array}{l}\text { Pressurized water. } \\
\text { GE }\end{array}$ \\
\hline $\begin{array}{l}\text { Submarine Intermediate Reactor } \\
\text { Mark A (DOE). West Milton, NY }\end{array}$ & SIG & & & 5500 & 5700 & Sodium. GE \\
\hline $\begin{array}{l}\text { Submarine Themal Reactor Facility } \\
\text { (DOE), NEL Site, ID }\end{array}$ & siw & & & 5300 & 8910 & $\begin{array}{l}\text { Pressurized water. } \\
\text { West. }\end{array}$ \\
\hline
\end{tabular}

\section{TEST AND RESEARCH}

\section{A. Test}

Aerospece Systems Test Reactor

(USAF), Fon Worth, TX

Ground Tea Reactor (USAF)

Fon Worth, TX

Nuclear Engineering Test Reactor

(USAF, Deyroa, OH

$\begin{array}{lllll}\text { ASTR } & 10,000.0 & 5400 & 7100 & \text { LWR. Convair } \\ \text { GTR } & 10,000.0 & 5300 & 7300 & \text { Pool. Convair } \\ \text { NETR } & 10,000.0 & 6500 & 7000 & \text { Tank Maxon-AC }\end{array}$

The TORY IC was successfully reated at full design power dering 5/64. Subsequent to crecellation of the Plue program 7/164, the reactor was placed in the Pluto disassembly building at NST for storage In 1974 the reactor was

transferred to the NERVA disascembly area for disassembly. 
3. TEST AND RESEARCH

PART II MUTARY REACTORS

\section{B. Research}

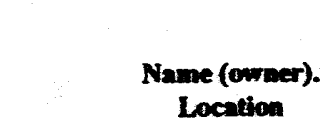

Amny Materials Reseanch Reactor

(USA). Regulated by NRC

Watenown, MA

Diamond Orduance Radiation Faciliry

(USA). Forest Glen, MD

Navil Research Reactor (USN).

Regulnod by NRC. Washington, DC

Nuclear Effects Reactor (DOE).

NTS, NV
Thermil Test Reactor No. 1 (DOE).

Schenectady, NY

Walter Reed Research Reactor (USA)

Regulated by NRC. Washington, DC

Designation
AMRR
DORF
NRR
Super KUKLA
TTR-1
WRRR

\section{POWER REACTORS}

\section{A. Central-Station Electric Power Plants}

Reactor Name (Owaer). Location
France (Franco-Belgian Society for Nuclear
Energy of Ardennes, SENA). Chooz
Germany, Kahl Nuclear Power Station
(Rhine-Westphadia Power Co., RWE).
Kahl-am-Main
Gernany (Kernkraftwert-RWE-Bayernwerk,
KRB1). Gundremmingen (near Gunzturg)
Italy. Garigliano Nuclear Power Statioa
(Project ENEL of SENN).
Punta Fiume (on Garigliano River)
Japan, Japan Power Demonstration
Reactor (IAERI). Tokai-Mura.
Ibaraki Pref.

NRC
export
license No.
rad date
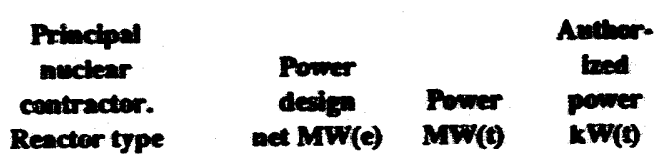

Initin

Simes

$\begin{array}{ll} & \text { West/fram, ACEC. } \\ & \text { Pressurized water } \\ \text { XR-031 } & \text { GE. } \\ \text { 0973059 } & \text { Boiting water }\end{array}$

$305.0 \quad 1,040.0$

0930/59

Boiling water

XR-052

05/28/64

XR-043

$08 / 16 \% 1$

GE

Boiling water

15.6

60.0

6700

9110

6100

8500

XR-045

GE

Boiling water

03/16/62

GE

Boiling water

$237.0 \quad 801.0$

$6700 \quad 8000$

64007800

$6300 \quad 8300$

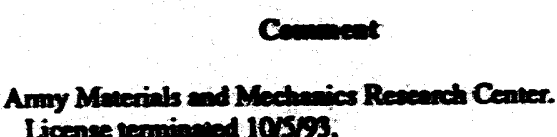

License terminend 105 ss.

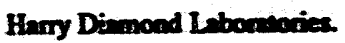

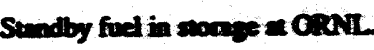

Whiver Reed Amy heximue of Recearch.

\section{B. Propulsion}




\section{TEST, RESEARCH, AMD TEACHMUG}

\section{A. General trradiation Test}

(No reactors currantly in this cregony)

\section{B. General Pesearch}

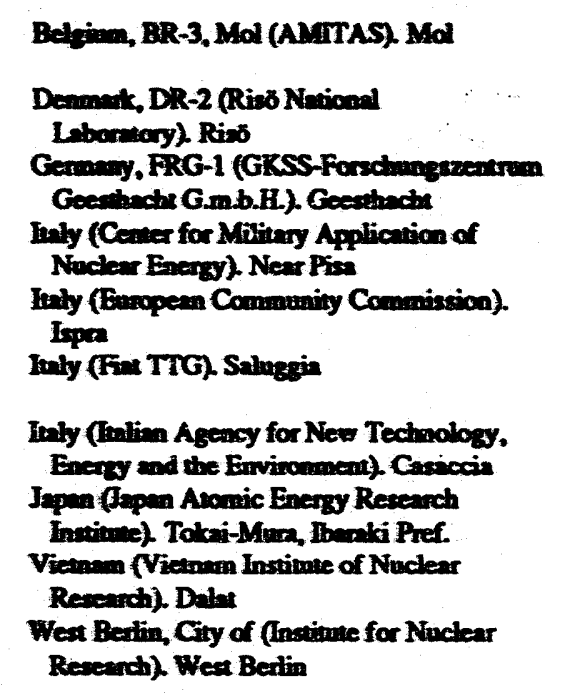

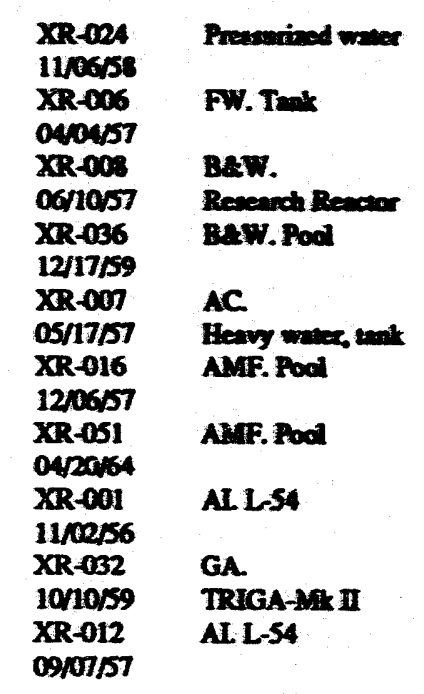

105

\section{Universty Research and Teaching}

$\begin{array}{lll}\text { Germany (Association for Radiation } & \text { XR-075 } & \text { GA. } \\ \text { Research). Munich } & \text { O1/14/71 } & \text { TRIGA-Mi II } \\ \text { Germany (Brown Boveri/Krupp). Jilich } & \text { XR-062 } & \text { AI L-77A }\end{array}$

Germany (Universities of Frenkfun and Damastade. Frankfiur

\section{XR-069}

$05 / 01 / 57$

AL. L-54

XR-022

$08 / 13 / 58$

AI. L-54

AGN.

AGN-201-111

Switzerband (University of Geneva).

Geneva

Unired Kingdom (Qveen Mary College.
London Universiny). London

XR-049

O2/13/64

AS Inc.

AS lnc.
UTR-B

$\begin{array}{rrr}5,0000 & 5800 & 7500 \\ 5,0000 & 5810 & \text { nd } \\ & 6300 & 8000 \\ 5,0000 & 5900 & 8900 \\ 7,0000 & 5900 & 7300 \\ & 7100 & 7900 \\ 500 & 5700 & 6900 \\ 2500 & 6300 & 7300 \\ 500 & 5800 & 7000\end{array}$

The rewetor contains some fiel and is still under

Ih i A juristiction.

Power: Pulaing.

Negligible power.

$\begin{array}{lll}1,0000 & 7200 & 8200 \\ & 6400 & 7700 \\ & & \\ 500 & 5800 & 6800 \\ 50.0 & 5900 & 8600 \\ & 5800 & 8700\end{array}$

This reactor was sold through Gulf Oil licensee, Guectofinengabune Sterterde A.E.

This L-7T reactor was opernied is the commercial exhibit of the 1958 Inemmional Confercace in Genewa mend in the USAEC Aloms for Peace Exhibits in Beinu, Leberaon, 10\%61; in Athens, Greece, 5/62, and in Baregkek. Thailand, 11/62.

Negligible power. The AGN-201-111 was opentod first in the USAEC Aloms for Peace Exhibit in Rome, Itly. 7/58, and later in the commercial exhibit of the 1958 International Conference in Geneva prior to transfer to the University of Geneva. 


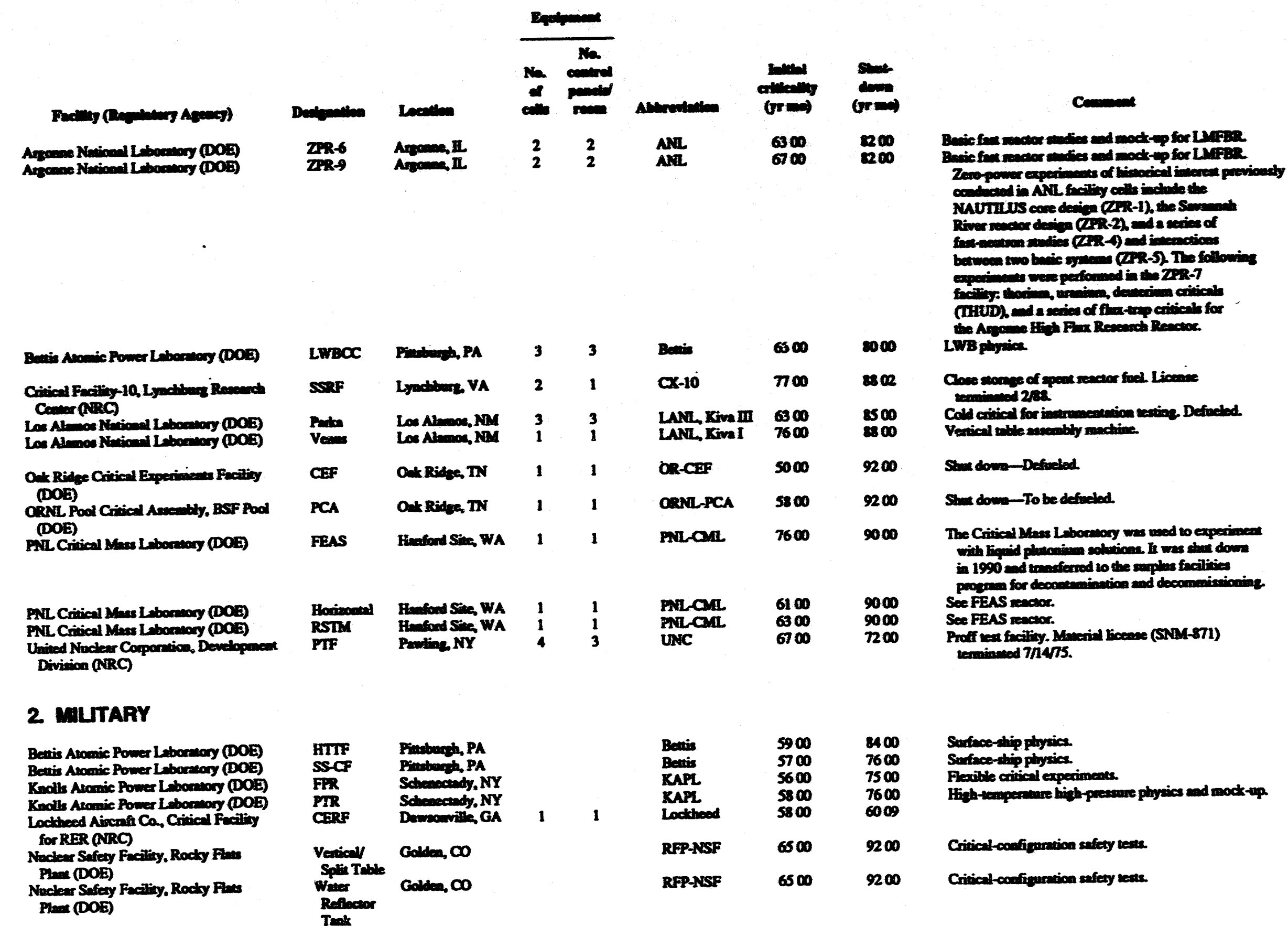


REACTOR INDEX 
Fictor masx

\section{AIw. 15}

ABenaM Lncoln

CVnm (2 reacoun) 14

Concon, 4

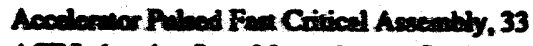

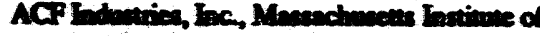

Tochalows: 9

$\operatorname{Aor}, 33$

ACa, 8

Adveced Evergy Revench betivete, Korea

IRTA-M H, 21

TIGA-Mx II, 2

Alvomed Newaicen Source Reactor, \&

Adrunced Reactivity Meamuemen Fecility 8

Advenod Tea Reactor, 7

Adveced Text Rescaor Critical Feciliny, 23

Aloviat-Genenal Conpontion

Geomad Experimeatel Engine Experiment XE-Bactrop, 30

XEPrive 30

Nuclear Rocked Engine Renctor Expeciment (NERVA)

NRX-A2, 30

$\operatorname{Nex}-13,30$

Tim -150

NoX-16-30

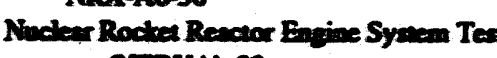
(NERYA) 30

\section{Aceoju-Ceneal Nideonio}

Areane National Labormory, 33

C.lifomin Polynechnic Sune Univexiny, 37

Cumclic Univesxidy of America, 37

Colondo Sune Univenity, 37

Delownes Univexiny of, 37

Ges Cocled Reacior Experimence, 43

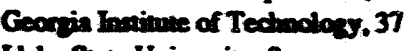

Who Sume University, 9

mally. 23

Kowea, 23

Memphis Sunce Univering, 38

Mabile Low Power Plasa No. 1, 43

New Merion, Univereity of, 10

Othboene, Universiny of, 38

Oregon Strme Univernity, 38

Polywochaic Inxituse of New Yot, 38

Swisteriond

Besel, Univervity of, 23

Geneva, Univexity of, 45

Texes ARM Univerity, 10

Twakegee Instione, 39
Unth Univexing of 39

Wea Vinimin Uhiverion. 3

Wilien Mand Rice Utivexioy, 39

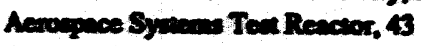

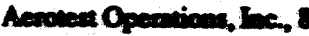

AFROI, 16

AFs, 34

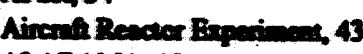

ALABAMA, 13

ALASKA, 13

Albain, 22

ALBANY, 14

AlBuQUEDQUE, 13

Aloo Protuces, Ine.

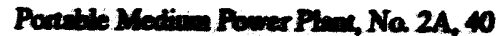

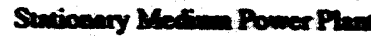
Na. 1,41 No. $1 A, 4$

ALEXANDER HAMIITTCN, 4

ALEXANDDIA, 14

Allis-Colbues Mis Co

Experimental Cos Coolad Reccetor, 28 Inly. 45

La Crose (Genen) Nuclear Generetion

$$
\text { Simion, } 25
$$

Nehartads, 20

Noclear Engimacing Teat Reactor, 43

Pubinder Alesic Fin 25

Souh Afrion, Sutani-1, 20

Swoden, 20

Almare

Unit 1, 18

Unit 2, 18

ALRR, 32

Alvin W. Vogle Noclear Ptum

Unit 1. 1

Unir 2,1

American Radimed

Andratin, Momen, 20

Japean, 23

Americen Simded the

American Stmeded Ince, 33

Iown Sime Univexing, 9

United Kingdemen 45

Virginin Palynechnic Inoimea, 39

Ames Laborwary Resenrch Reacior, 32

AMF Alomics, he.

Ames Leborntory Rescench Reactor, 32

Anseria, Asen, 20

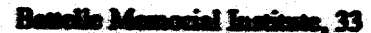

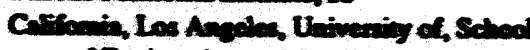

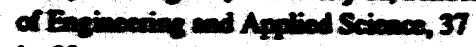

Comin, 2

Cimidion be Rewer, 32

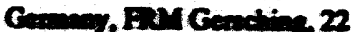

Cresce, Demecritar, 21

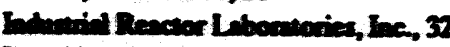

$\operatorname{lom}, 23$

Ianel 24

inly

Cuscocin, 45

Silnain, 45

I. In-2,20

Monmecollepes

Nebeinen, 23

$\mathrm{New}$ Yate, Sunc Univening of, 10

Nonh Conclim Stue Univerien, 10

Putionar, PNRR, 21

Penger, DP1, 21

Tudrey. 21

Wabtingen, Univenioy of, 39

AMTAS, Betyine, Bie-3, Mol, is

Ama 44

ANDEEW IACESON, 4

Angen 1, Cencel Electicia de Fuman, 16

ANNAPOLI, 14

Anomler Cone Pulad Reacior, 33

ANs, 8

APFA-III,33

APRF, 16

ARCHEDFEH, 12

ARE, 43

Arpanour (CP-11) 33

Arponec CP-3, newiin as CP-3. 33

Argone Low Power Reseanch Reacuor, 33

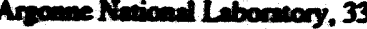

Agrane Low Power Recench Reacex, 33

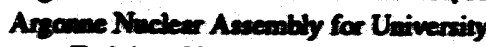
Tniniog 33

Ageme Research Reactor, 32

Argane Treand Senpe Reactor, 33

Biclogional Resenuch Reacior, 33

Boitine Rewesor Experimean No. 1, 27

Boiling Renctor Experiment No. 5, 27

Bolling Reactor Experiments, 27

Experimentel Breeder Reactor No. 1, 18

Experimeanel Breeder Reactor II. 6

Experimened Boiling Water Reactor, 27
Fom Somon Reneres 34

Vlob Dinition, 2PSh, 23

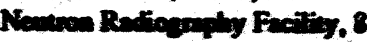

Somionny Low Power Fen Na 1, 4

Trumien Beactor Tex 7

T28, 46

Z2R-9, 4

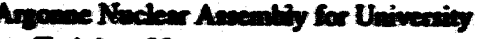

Triming 33

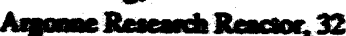

Apome Theosed Sowce Reactor, 33

Arinose, Univering of, 9

Aribena Public Senvice Co.

Plo Verde Nrateor Cenernies Sumion

Unim 1 3

Unie 2,3

Unie 3,3

ADKANASAS (2 remons) 14

Alomean Nuctear One

Unin 1, 1

Uni 2,1

Atreanes Tech Univening, 11

Aned Forces Radiobiolosy Reseanch loxtinue

DNA, 16

ARMF, 8

Anny Merviels and Mechanics Reseanch Cener.

Anny Mererinds Reweanch Reactor, 44

Army Pule Radinion Faility, 16

Amold, Dusme Eserny Center, Unia 1.2

ARR (L-54), 35

Asco

Unit 1, 18

Unin 2, 18

ASHEVILE, 14

ASPRO, 12

Ascocined Universinies, Inc.

Broolthaven Graphie Research Reacior, 33

Brocldonven Newtron Source Reacao Na. 1, 34 No. 2, 34

ASTR, 43

Antan, 20

Aveliers de Conotnetions Electriques, de Charleroi S.A.

Belojiem, Thanese, Unit 1,16

France, 44

ATLANTA, 13

Alomics Intemational, 33

AIR, 7 


\section{PEAGTOR MBSX (Contmuad)}

Arco 2

ATs:33

AUCOSTA, 13

Ansmis

$\operatorname{Man} 20$

Amain

Ano, 20

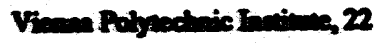

\section{B Reacos, 39}

Babcoct \& Wrout Ca

Atomen Iteder One, Unit 1,

Bellefones Nucleor Fino

Unit 1,5

Unit 2,5

Benet22

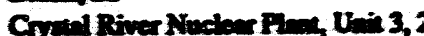

Drvid-Beace Nodber Power Sunion, Uhin 1,2

Cocmany, F20-1, 45

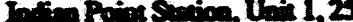

ming, 45

Michion, Uhivening of foud Rivelen Beactorit, 9

Comen Necleur Simin

Unit 1,3

Unin 2,3

Unie 3,3

These Milie Xlend Nocien Sumion

Unit 1.5

Uni 2,26

Vinginin, Univeniny of, 10

Wabingea Nuclaer Project, Unit 1,5

Baboock \& Wiloen

Lynchiburs Pool Reacter, 33

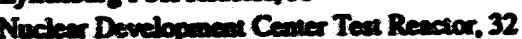

BAPBRIDCE (2 reactoxi), 14

BALTM $O R E, 13$

Berimore Ges A Electic Co.

Calven Crifis Nuclear Power Plon

Unit 1, 1

Unit 21

Bendedech, 20

BARB, 41

Baed, Univerity of, Switratend, 23

BATFEH, 12

BATON ROUGE, 1

Bmelle Memorial lextimes 33

BAwTR, 32

Beaver Valley Power Sumion
Das, Mal, is

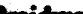

Unis 1,1

$\operatorname{lon} 21$

Bient

Amere 1, Cond Decticie de Rum, 16

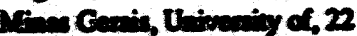

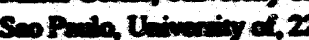

Buangion, 13

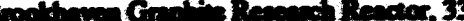

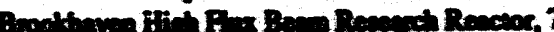

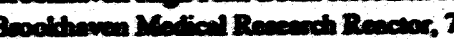

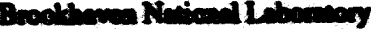

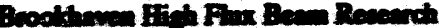
Revecor, 7

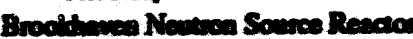

No. 1,34

Na. 2, 34

Fon Vhmar Somed Rencor, 3

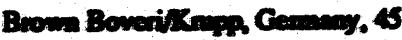

Boowm Fery Nocleor Power Sinio

Unit 1. 1

Unit 2, 1

Unie 3,1

BDR, 33

Brmaswict Sten Electic Phat Unit 1, 1

Unin 2,1

BSe, 34

BUFFALO, 13

Buffilo Mentinls Revench Cener, Sume

Unives of New Yout 10

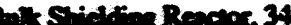

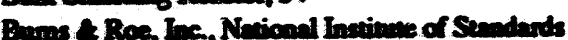
a Technolosy. 7

Brace Sutica

Unie 1, 1

unin 2

CReactor

$$
\text { Aiven, } 39
$$

Bichlond, 39

Crlhoon, Fon, Sunion, Unit 1, 2

CALIFOENIA (2 reactore), 14

Colifomia

Berteloy, Univerity of, 37

Irvine, Univening of, 9

Las Anedes, Univesing of, School of

Enginceain and Applied Scioncen, 37
Colonkin IMNE1, 20

Colondo Sum Univention 37

colusme, is

Colmovin Univesing, 37

Counnus, 14

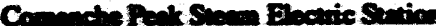

Vuin 1,2

$\operatorname{Unin} 22$

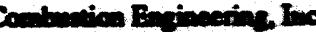

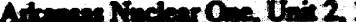

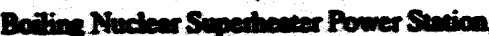
25

Colven Crefis Nindeer Power Plum Unis 1,1

Unie 2,1

Fon Collow Sento

Uhin 1.2

seane

Uldiin 3, 19

Ulahin 4.19

Yomenwane 4, 19

Yomeswent-3, 1

Mrine Y ahee Alowic Power P inn

Metratone Nuclear Power Stration, Unit 2, 3

Polienden Nucloen Ptom Unit 1,3

Palo Vende Nuclear Genereting Sinio Unit 1,3

Unin 2,3

Unit 3, 3

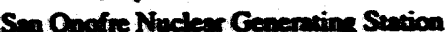

Unie 2.4

Unir 3,4

St Lucie Pinat

Unit 1.4

Unim 2.4

Wathingen Nuclear Projoct, Unit 3,5

Weterfod Cenenting Sinion, Unit 3,5

Comes, 23

Commenventh Edisea Ca.

Brnidwood Sintion

Unit 1, 1

Unie 2, 1

Byron Simion

Unin 1,1

Unin 2, 1

Dredea Nuclear Power Station

Unit 1.25

Unit 2,2

Unit 3,2
Cons. 4

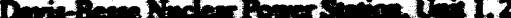

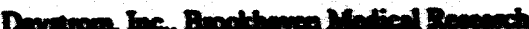
Reacher, 7

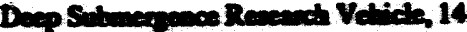

Delowere Univenioy of, 37

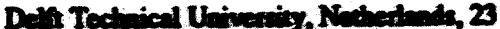

Demonention Reacter, 34

Demorition, 21

Denont, D2-1.20

Deamenc, DR-2, 4

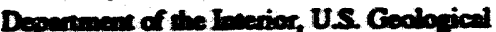

Servey L Lbentosy. 8

Demoyer Reador Prowspes is

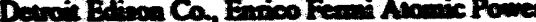
PInt, Uni 2,2

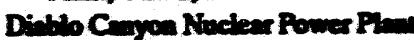

Unit 1,2 Unie 2,2

Dirmond Ondanese Radimion Fecitiny. 44

DNA Depnoment of Deforen (DOD). Amed

Forces Rediobiolosy Resench botimite, 16

Dodewand, 17

DOE Demonetrotica Reactor, 34

Doel

Unit 1, 16

Unit 2,16

Unin 4, 16

Dorald C. Cook Nuclent Power Plone

Uni 1,2

Unir 2,2

DORF, 4

Dow Cremical Co, 8

DR Reacter, 39

DR-1, 20

DR-2, 4

DREADNOUGHT, 44

Dreadea Nuclear Power Sintion

Unit 1.25

Unin 2,2

Unit 3,2

DRUM, 12

Du Pone de Nemours \& Company, Inc., EI.

B Reacior

C Resceor, 39

D Reactor, 11

Experiment Cosmplex, 40

F Reactor, 39

Heafoed 305 Tear Reactor, 40 
Unis 1,1
Unit 2,1

Belaiven

BR-3, Mal, 45

Doel

Unia 1, 16

Unin 2, 16

Unit 4, 16

Tribenge

Unit 1.16

Unit 3, 16

Bellefonie Nuclear Phane

Unit 1,5

Unit 25

Bendix Avintion Conp.

Amy Mmerinls Resenrch Reactor, 44

Kenses, Univertity of, 38

BENIAMIN FRANKIN,

BERGALL, 12

Bemieche Knfrwerke AG, Switzertend,

Mubleberg. 18

Bethleben Steel Co., Shipbeilding Division

USS BAINBRIDGE (2 reactors), 14

USS LONG BEACH (2 reacion), 14

$$
\text { HTTF, } 46
$$

LWBCC, 46

SS-CF, 46

Bernm

Unit 1, 18

Unit 2, 18

BGRR, 33

Bis Rock Point Nucles PImet, 1

Big Ten, 23

BILLFSH, 12

Biological Resentch Reacior, 33

BIRMINGHAM, 13

Bhw-Knox Co., Meterials Tenieg Renctor, 31

BLUEFISH, 12

BMRR, 7

Boilieg Nuclear Supentedter Power Sunion, 25

Boiling Reactor Experimend

No. 1, 27

No. 5,27

Boiling Renctor Experiments, 27

BOISE, 14

BORAX reactors, 27

BOSTON, 13

Bowan Edison Co. Plarim Nuclen Power Sution, Unit 1, 4

Semen Brten, Universiay of, 37

Califorain Polytechnic Stute Universiny, 37

Callmay Plen, Unis 1, 1

Cilven Clifis Nuclear Power Plane

Unit 1, 1

Unit 2, 1

Cranda, MeMater University, 22

Cocreo Nuclear Station, 17

CARL VINSON (2 reactors). 14

Carolima Power a Light Co.

Brunswict Stent Electric Plent

Unit 1.1

Unit 2, 1

H.B. Robiascon Plana

Unit 2,2

Shearon Harris Nuclear Power Plunh, Unin 1,4

Carolinas-Vinginin Tube Reactor, 25

CASTMIR PULASKI, 42

Catambe Nuclear Sumion

Unit 1,1

Unit 22

Crubolic University of America, 37

CAVALIER, 39

CaYalla, 13

CEF, 46

C.EN., Manmore., Monocco, 22

CERF, 46

CFRMF, 8

CHARLOTTE, 15

CHEYENNE, 15

CHCAGO, 13

Chicago Pile 1, rebuilt as CP-2, 34

China, Republic of, 22

Chinatum

Unia 1,18

Unit 2,18

CINCMNATI, 13

CINR, 32

Cintichem, Inc. Reacear, 32

CTYY OF CORPUS CHRISTI, 13

Cevelend Electric Iluminatias $\mathrm{Co}_{0}$

Perny Nuclear Power Plan

Unit 1.4

Unit 2,5

Cinton Leboratory, Oak Ridge Graphite

Reacior, 36

Chimen Power Simion, Unit 1.2

Cofremes, Unin 1, 18
La Solle Comary Sumion

Unit 1.3

Unit 2,3

Quad Covies Suction

Unit 1,4

Unie 24

Zion Nuciear PInat

Unin 1,5

Unit 25

CONNECTCUT, 15

Connecticut Yankee Avemic Pomer Ca, Haddem Nect Plimen, 2

Conatidned Ediven Ca of New Yoak, lne

Indien Poin Semion, Unih 1.25

Indian Point Sution, Uain 2,2

Consumen Power Co.

Bie Rock Point Nuclear Fl-1 1

Palisades Nocleer Ptex, Unit 1,3

Covenumess Public Pomer Dimsict, Hellom

Neclear Power Feciliny, Strebion Suntion, 25

Cook. Dowald C. Nuclear Power Pint

Unit 1,2

Unit 2, 2

Cook Electric Compray, North Camalina Stute

University, 38

Cocper Nuclear Simion, 2

Comell Universing, 9

Comell Universiny Zexo Power Reactor, 9

Coupled Fast Reactivity Measurement Facility, 8

CP-2,34

CP-3, 33

CP-5, 32

Cyyud River Nuclear Fines, Unit 3,2

Curtiss-Wrigh Cosportion, Missouri at Rollh, University of, 9

Cunias-Wright Noclear Recearch Lobordory of

the Commonwealth of Penosylvenin, 34

CWRR, 34

CX, 23

DRenctor, 39

DIG, 15

DACE, 42

Dairyland Power Cocperenive, La Crosece (Gemon)

Nuclear Gesenting Suction, 25

DALLAS, 13

DANIEL BOONE, 12

DANIEL WEBSTER, 12

Darmesad, Universities of Fronkfiun and.

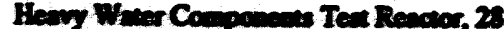

KReacter, 39

LReacor, 39

Levice Tear Remelor, 40

P Rewerer

Procens Dovelonent Pile, of

R Reactor, 39

SR 306-M Tea Prie, 40

Standad Files burciticel

Drume Arsoid Exent Coner, Unit 1.2

Duke Power Co.

Crumbe Naclem Sution, Unit 2,2

Oconse Nucleer Sumien

Unil 1,3

Unit 2,3

Unit 3.3

Williz B. MoGnire Neclear Sutio

Uni 1.5

Unit 2,5

Durneane

Bemer Valley Power Sution, Unin 2, 1

Ohio Edisca Co., Benver Valley Power Stration, Unit 1, 1

Shippingpon Atomic Power Stmica, 26 DWIGHT D. ETSENHOWER (2 remerom) 14

EAEP. 34

EBOR, 27

EBR-1.28

EBR-II, 6

EBWR, 27

Etucaner

Celifomin, Los Angeles, Univeriity of. School of Engimoering and Applied Science, 37

Wathingen, University of, 39

Edwin I. Hach Nuclem PI

Unit 1,2

Unit 2,2

ECreG ldabo, lace.

Advancod Reactivity Measurement Fucility, 8

Adruaced Teat Reactor, 7

Coupled Fasa Reactiviny Meanemen Facility. 8

Loes of Fmid Tert 32

Power-Bunt Facility. 32

ECCR, 28

Ell Rive Reacsor, 25

ENEI, haly 
Caorso Nuclear Sintion, 17

Trimo Vercellese, 17

Enerny Center, Nethertands, 20

Engineerine Tear Reactor, 31

Englind

Imperial Chemical Industries, 20

Sizewell B, 19

ENHER, Spain, Vandellos, Unit 2, 18

Enrico Fermi Alomic Power Plant

Unit 1,25

Unit 2,2

Entergy Operations Inc.

Arkansas Nuclear One

Unit 1, 1

Unit 2,1

Grand Gulf Nuclear Station, Unit 1,2

Waterfond Generating Station, Unit 3,5

ENTERPRISE (8 reactors), 14

EOCR, 28

ESADA Vallecitos Experimentul Superheat Reactor, 27

ETHAN ALLEN, 42

ETR, 31

4. European Commusity Commission, Italy, 45

European-Asian Exhibit Program, 34

EVESR, 27

Experimental Beryilium Oxide Reactor, 27

Experimental Boiling Water Reactor, 27

Experimental Breeder Reactor No. 1,28

Experimental Breeder Resctor II, 6

Experimental Gas Cooled Reactor, 28

Experimental Organic Cooled Reactor, 28

Experimental Propulsion Test Reactor

TORY IIA, 43

TORY IIC, 43

F Reacior, 39

Friley, Joseph M., Nuclear Plant, Unit 1, 3

Unit 2, 3

Fast Burst Reactor Facility, 16

Fast Flux Test Facility, 31

Fast Neutron Source Reactor, 34

Fast Source Reactor, 34

FBRF, 16

FCPE, 24

FECSA, Spain, Asco

Unit 1,18

Unit 2, 18

Fermi, Enrioo, Atomic Power Plant
Gariglimo Nuclear Power Station, 44

Gas Cooled Reactor Experiment, 43

GATO, 12

GCRE, 43

General Alomics Technologies, 34

General Aromics Technologies

Accelertor Pulsed Fast Critical Assembly, 33

Advanced TRIGA-Mk F Prototype Reactor, 8

Aeroless Operations, Inc., 8

Albania, 22

Annular Core Pulsed Reactor, 33

Arizona, University of, 9

Arkansas Tech University, 11

Amed Forces Radiobiology Research

Instimite, DNA, 16

Austria, 22

Bangladesh, 20

Bravil, 22

California

Berikeley, University of, 37

Irvine, University of, 9

Columbia University, 37

Comell University, 9

Diamond Ordnance Radiation Facility, 44

Dow Chemical Co., 8

England, 20

Experimental Beryllium Oxide Reactor, 27

Finland, 22

Fon St. Vrain Nuclear Generating Station, 25

Cermany

Hanover, Medical College of, 22

Mainz, Johannes Gutenberg University of, 22

Nuclear Medicine, Institure for, 22

Rediation Research, Association for, 45

Mlincis, University of, 9

Indonesia

$$
\text { Bandung. } 21
$$

$$
\text { Yogyakarta, } 21
$$

Inly

IRIGA-Mk II, XR-026, 21

TRIGA-Mk II, XR-056, 23

Japan

NSRR, 20

TRIGA-Mk II, XR-037, 23

TRIGA-Mk II, XR-038, 23

Kenens State University. 9

Korea

TRIGA-Mk II, XR-027, 21
BFRGAU 12

BILLFISH, 12

BLUEFTSH, 12

BOSTON, 13

BREMERTON, 13

CASMAIR PULASTK, 42

CaVALLA, 13

CITY OF CORPUS CHRISTI, 13

COLUMBLA, 15

CCLUMBUS, 14

CONNECTICUT, 15

DALLAS, 13

DANIEL WEBSTER, 12

Deep Submergence Recearch Vehicle, 14

ETHAN ALLEN, 42

FLASHER, 42

FLORIDA, 13

FLYNG FISH, 12

FRANCIS SCOTT KEY, 42

GEORGE BANCROFT, 42

GEORGE WASHWNGTON, 41

GEORGIA, 13

GLENARD P. LAPSCOMB, 42

GROTON, 13

HARTFORD, 15

HELENA, 13

HENRY L. STIMSON, 4

HENRY M. JACKSON, 13

HYMAN G. RICKOVER, 13

ENDIANAPOLIS, 13

JACKSONVILLE, 13

JAMES K. POLK, 12

KENTUCKY, 13

LA JOLLA, 13

LAFAYETTE, 42

LOUISIANA, 15

LOUISVILLE, 13

MAINE, 15

MARYLAND, 13

MIAMI, 14

MICHIGAN, 13

MINNEAPOUS-SANT PAUL, 13

NARWHAL, 12

NATHAN HALE, 42

NEBRASKA, 13

NEVADA, 13

NEW YORK CITY, 13

OHIO, 13

OMAHA, 13
Unit 1,1

Unit 2, 1

CRewer, 39

China, Republic of, 22

Chimen Power Sintion Unit 1,2

Cocper Nuclear Station, 2

Destroyer Reacuce Prosotype, 15

DR Remetor, 39

Dresdin Nuclear Power Station

Unit 1, 25

Unit 2, 2

Unit 3,2

Drane Amold Enerry Center, Uniz 1,2

Edwin I. Histch Nuclem Piant

Unit 1,2

Unit 2,2

Enfincering Tea Reactor, 31

Enrico Fermi Alomic Power Pland Unil 2, 2

ESADA Vallecilos Experimental Superteat Resctor, 27

General Electric Nuclear Ten Reactor, 8

Germany

Kahl Nuclear Power Station, 44

Kemknefiwerk-RWE-Beyemwerk, KRBI, 44

Grand Gulf Nuclear Sintion, Unil 1, 2

H Reactor, 39

Hope Creek Nuclear Generating Sution,

Unit 1,2

Humbolds Bay Power Plant, Unia 3, 25

India

Tarapur Nuclear Power Sontion

Unit 1, 17

Unit 2, 17

Italy

Coorso Nuclear Suntion, 17

Garigliano Nuclear Power Station, 44

James A. FitzPatrick Noclear Power Plant, 3

Jopan

Fukushims Dai-ichi Power Sation

Unit 1, 17

Unit 2, 17

Unit 6, 17

Japin Power Demonstration Renctor, 44

Kashiwazaki-Kariwa

Unit 6, 19

Unit 7, 19

Tokai No. 2 Power Station, 17

Tsuruga Power Sestion, Unit 1, 17 


\section{Unit 1,25}

Unit 2,2

FFTF, 31

Fine TTG, Inly, 45

FIBACK, 12

Finderd lantimue of Technolosy, 22

Fin Pariat, James A., Nuclear Power Plane, 3

FLASHER, 42

Flanop, 23

FLORIDA, 13

Florich, University of, 9

Foridh Power \& Lights Co.

S. Lucie Pinnt

Unil 1,4

Unit 2,4

Turkey Point Plant

Unis 3,5

Unit 4,5

Forids Power Copp, Crystal River Nuclear Plant, Unit 3, 2

Furor Corportion, LLd, Experimentel Organic Cooled Reactor, 28

FLYING FSH, 12

FNR, 9

$\because \quad$ Fond Nuclear Renctor, Michigm,

$$
\text { University of, } 9
$$

For Calboun Sution, Unit 1, 2

Fon SR. Vrain Nuclear Generating Stution, 25

Fonter Wheeler Corp.

Denmart, DR-2, 45

Livemore Pool Type Reactur, 35

FPR, 46

Framatome

Tihange, Unit 1, 16

Funce, 44

FRAN, 35

Frence, Franco-Belgien Society for Nuclear

Energy of Ardennes, SENA, 44

FRANCIS SCOTT KEY, 42

Frankfun and Darmstudt, Universities of.

$$
\text { Germany, } 45
$$

$$
\text { FRG-1, } 45
$$

FRM Gersching, 22

Fual Element Tea Bed, 30

Fuel Element Test Reactor

$$
\text { Pewee-1, } 30
$$$$
\text { Pewee-2, } 30
$$

Fukushima Dai-ichi Power Suation

Unit 1, 17

Unit 2, 17

Unit 6, 17

\section{TRIGA-Mi II, XR-073, 21}

Malayaia, 21

Marylad, Univenity of, 9

Mexico, 21

Mictigan State Univeriny, 38

Morocesa, 22

Northrop Corporate Luboratories, 35

Omath Veterms Adninistration Hospital, 8

Oregon Stuce Univerrity, 10

Peach Bonom Alomic Power Stution, Unit 1. 25

Pean Stute TRIGA Rewator, 10

Philippines, Republic of the, PRR-1, 21

Puerto Rico Nuclear Ceater, 38

Reed College, 10

Romania

TRIGA (MPR 10), 20

TRYGA-ACPR, 20

Slovenia, 21

Sintionary Newuron Radiography System, 16

Teras as Austin, University of

TRIGA-MK I, 39

TRIGA-MR II, 10

Thailend, TRR-1, 21

TRIGA-MKI Protoispe Reactor, 8

Tustey, 21

US. Geological Survey Laboratory, 8

Unh, University of, 10

Vietnam, 45

Washington State Utriversiry, 1

Wisconsin, Univensity of, 11

Zaire, 22

Genesl Dynamics Corp.

Convair Divisio

Aerospace Systems Test Reactor, 43

Ground Teat Reactor, 43

Electric Bont Division

ABRAHAM LINCOLN, 41

ALABAMA, 13

ALASKA, 13

ALBUQUERQUE, 13

ALEXANDER HAMILTON, 42

ALEXANDRIA, 14

ANNAPOUIS, 14

ARCHERFISH, 12

AUGUSTA, 13

BALTIMORE, 13

BATFISH, 12

BENJAMIN FRANKLIN, 42
PARCO, 12

PASADENA, 14

PATRICK HENRY, 41

PENASYVANI, 13

PLIILADELPHIA, 13

PHOEND, 13

PITTSBURGH, 13

PORTSMOUTH, 13

PROVIDENCE, 13

RHODE ISLAND, 15

SAN JUAN, 14

SANTA FE, 14

SCORPION, 41

SEA HORSE, 12

SEAWOLF PWR, 41

SEAWOLF Sodimm Reaceor, 4

SEAWOLF, 15

SILVERSTDES, 12

SKATE, 41

SXPIACK, 41

SPRINGFIELD, 14

STURGEON, 12

TECUMSEH, 42

TENNESSEE, 13

THOMAS A. EDISON, 42

TOPEXA, 14

TREPANG, 12.

TRITON (2 Reactors), 41

TULLIBEE, 41

ULYSSES S. GRANT, 42

WEST VIRGINIA, 13

WYOMING, 15

Quingy Division

GATO, 12

GREENLING, 42

SUNFIS, 12

WHALE, 12

General Electric Company

ANPD

Heat Transfer Reactor Experiment

No. 1,43

No. 2, 43

No. 3, 43

Bis Rock Point Nuclear Pleat, 1

Browns Ferny Nuclear Power Station

Unit 1. 1

Unit 2,1

Unit 3.1

Brunswick Steam Electric Plant
KB Reactor, 39

La Sulle County Stution

Unit 1,3

Unit 2, 3

Lelend Sinford University, 38

Limedick Generning Sution

Unit 1,3

Unit 2,3

Messechosens, University of, 9

Mexico, Lopuna Verde Strion

Unit 1, 17

Unit 2,19

Millutane Nuclear Power Station, Unit 1, 3

Modificmions and Additions to Reactor Fecility, 15

Monticello Nuclear Genernting Plant, 3

Neaheriands, Dodewand, 17

Nine Mile Point Nuclear Station

Unit 1, 3

Unit 2, 3

Oysuer Creek Nuclear Power Plant, Unit 1, 3

Peach Bottom Atomic Power Station

Unit 2,3

Unit 3,3

Perry Nuclear Power Plent

Unit 1, 4

Unit 2,5

Pilgrim Nuclear Power Simtion, Unit 1, 4

Quad-Cities Station

Unit 1, 4

Unit 2,4

River Bend Station, Unit 1 , 4

Shoreham Nuclear Power Siation, 26

Small Submarine Reactor Protokype, 43

Southwest Experimental Fast Oxide Reactor, 29

Spain, 21

Cofrentes, Unit 1, 18

Santa Maria de Garona, 18

Valdecaballeros

Unit 1,19

Unit 2, 19

Submarine Advanced Reactor Prototype, 43

Submarine Intermediate Reactor Mark A. 43

Susquehanna Steam Electric Station,

Unit 1.5

Unit 2,5

Switzerland

Leibstudt, 18 


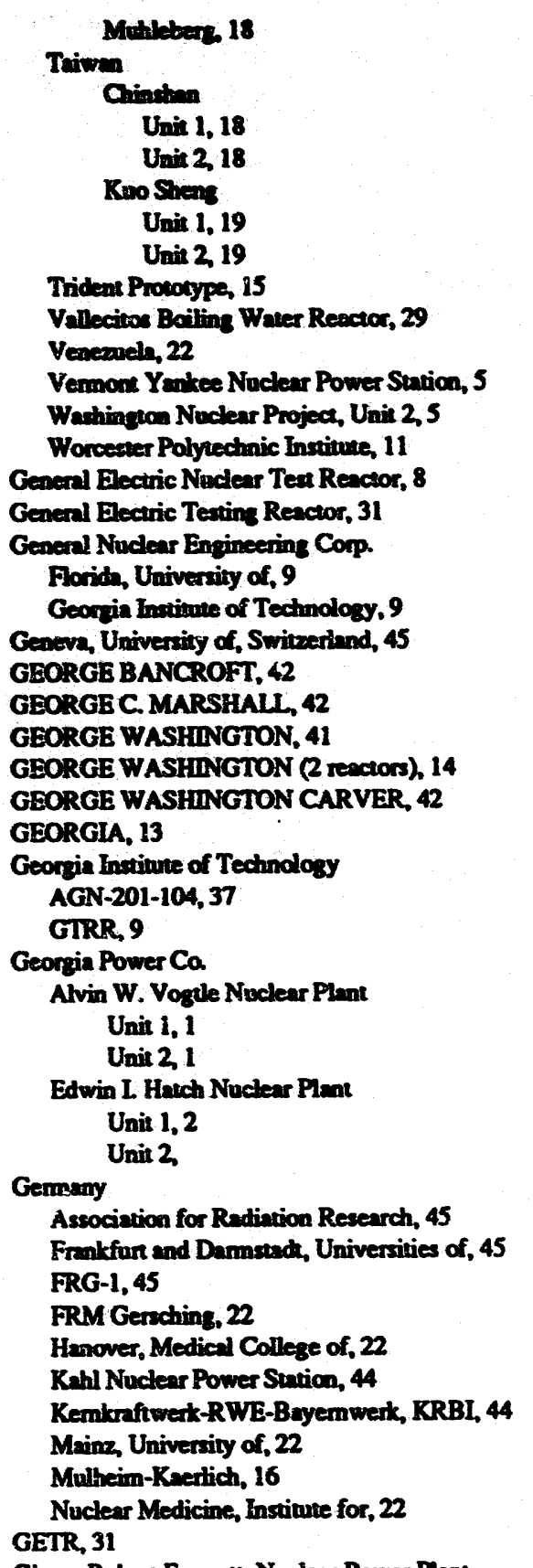

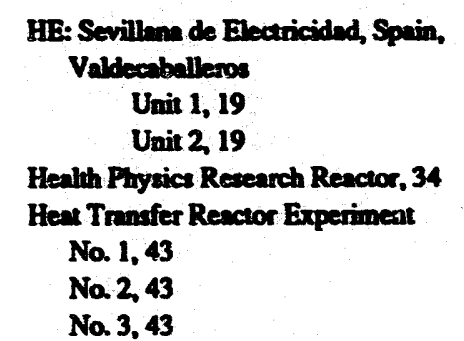

Heavy Water Componemes Text Reactor, 28

HELENA, 13

HENRY CLAY, 42

HENRY L STIMSON, 42

HENRY M. JACKSON, 13

HFBR, 7

HFIR, 7

Hidroelecerica Espanola S.A., Spain, Cofrentes, Unit 1, 18

High Tempermure Latice Test Reactor, 34

Hitachi, Japan

Kashiwazaki-Kariwa, Unit 7, 19

Tokai No. 2 Power Sution, 17

Homogeneous Renctor Experiment

No. 1,28

No. 2,28

Honeycomb, 23

HONOLULU, 13

Hope Creek Nuclear Genenting Station, Unit 1, 2

Horizontal, 46

Horizontal/Split Table, 24

HOUSTON, 13

Houston Lighting \& Power Co., South Texas Project

$$
\text { Unit 1,4 }
$$

Unit 2, 4

HPRR, 34

HRE-1, 28

HRE-2, 28

HTLTR, 34

HTR, 40

HTRE-1, 43

HTRE-2, 43

HTRE-3, 43

HTTF, 46

Humboldt Bay Power Plant, Unit 3, 25

HWCTR, 28
Irm, University of Tehron, 23

IRL, 32

Invel, Atomic Energy Commission, 21

Inily

Cacro Nudear Station, 17

Center for Military Application of Nuclear Enerzy, 45

Enropen Community Commiasion, 45

Fint TTG, 45

Garigliano Nuclear Power Sution, 44

Inlien Agency for New Technology. Energy and the Environmen$$
\text { Pool, } 45
$$

TRIGA-Mk II, 21

Milan, University of, 45

Palermo, University of, 23

Pavia, University of, 23

Trino Vercellese, 17

JACK, 41

JACKSONVILLE, 13

JAERI, Japen, Japen Power Demonstration Reactor, 44

Jamaica, Kingston, 21

James A. FitzPatrick Nuclear Power Plant, 3

JAMES K. POLK, 12

JAMES MADISON, 42

JAMES MONROE, 42

JANUS, 33

Japan

Fukushima Dai-ichi Power Sution Unit 1, 17 Unit 2, 17

Unit 6, 17

Japen Atcmic Energy Research Institute, 45 Japen Power Demonstration Reactor, 44

JRR-2, 20

Kashiwazaki-Kariwa

$$
\text { Unit 6, } 19
$$$$
\text { Unit 7, } 19
$$

Kinki University, 23

Mihsma Power Station, Unit 1, 17

Musashi College of Technology, 23

NSRR, 20

Ohi Power Station

$$
\begin{aligned}
& \text { Unit 1, } 17 \\
& \text { Unit 2,17 }
\end{aligned}
$$

Rikkyo University, 23
Kivi-B1A, 31

Kiwi-B1B, 31

Kiwi-BAA, 31

Kivi-BAD, 31

Xiwi-BAE, 31

Kiwi-Tnnsient Teat Reactor, 32

Kiwi-TTR, 32

Knolls Atomic Power Labormory

FCPE, 24

FPR, 46

PTR, 46

Thermal Tea Reactor No. 1, 4

Korea

Advanced Energy Resenrch Institute TRIGA-Mk II, 21 TRIGA-Mk II, 21

Kori-1, 17

Kori-2, 17

Kori-3, 17

Kori-4, 17

Kyong Hee, University of, 23

Ulchin 3, 19

Ulchin 4, 19

Yonggwang-1, 17

Yongewang-2, 17

Yonggwang-3, 19

Yongswang $-4,19$

Koni-1, 17

Kori-2, 17

Kori-3, 17

Kori-4, 17

Krsko, 18

KUKLA, 35

Kuo Sheng

Unit 1, 19

Unir 2, 19

KW Reactor, 39

Kyung Hee, University of, Korea, 23

L Reactor, 39

L MENDELL RIVERS, 13

La Crosse (Genoa) Nuclear Genernting Sution, 25

LA JOLLA, 13

La Salle County Station

Unit 1,3

Unit 2,3

LAFAYETTE, 42 
CXN, Netherlands, Dodewand, 17

one Forschungezentrum Geesthacht G.m.b.H., Yermany, FRG-1, 45

GLENARD P. LIPSCOMB, 42

Godiva-IV, 23

GPU Nuclear Conp

Oyster Creek Nuclear Power Plant, Unit 1,3

Three Mile Island Nuclear Station

Unit 1,5

Unit 2,26

Grand Gulf Nuclear Station, Unit 1,2

GRAYLNG, 12

Great Britain, SSW for HMS DREADNOUGHT, 44

Greece, Democritos, 2

GREENLING, 42

GREENVILLE, 15

GROTON, 13

Ground Experimental Engine Experiment

XE-Burkup, 30

XE-Prime, 30

Ground Test Reactor, 43

GTR 43

॥ GTRR, 9

GUARDFISH, 42

GUTTARRO, 42

Gulf States Utilities Co., River Bend Station,

Unit 1,4

GURNARD, 12

H Reactor, 39

H.B. Robinson Plant, Unit 2.2

Heddam Neck Plent, 2

HADDO 41

HADDOCK, 42

HALIBUT, 41

Hallam Nuclear Power Facility, Sheldon Station,

\section{HAMMERHEAD, 12}

HAMPTON, 14

Hanford 305 Test Reactor, 40

Hanover, Medical College of, Germany, 22

Herris, Shearon, Nuclear Power Plane, Unit 1, 4

Harry Diamend Laboratories, USA, Diamond

Ordnance Radiacion Facility, 44

HARTFORD, 15

Hatch, Edwin L., Noclear Plant

Unit 1,2

Unit 22

HAWKBIL, 12
HYMAN G. RICKOVER, 13

HYPO, 35

IAN-R1, 20

Idaho Nuclear Corporation, Special Power

Excursion Reactor Test No. 4, 33

Idabo Stale University, 9

Ilinois, University of,

LOPRA, 9

TRIGA-Mk II, 9

minois Institure of Technology Research Institute, 35

minois Power Co., Cinton Power Station, Unit 1,2

Imperial Chemical Industries, England, 20

India, Tarapur Nuclear Power Station, Unit 1, 17 Unit 2,17

Indian Point Station

Unit 1, 25

Unit 2, 2

Unit 3,3

Indiana and Michigan Eloctric Co., Donald C. Cook Nuclear Power Plant

Unit 1,2

Unit 2, 2

INDIANAPOLIS, 13

Indonesia

Bandung. 21

Yogyakania, 21

Industrial Reactor Laboratories, Inc., 32

Ingalls Shipbuilding Corp.

ASPRO, 12

BARB, 41

DACE, 42

HADDOCK, 42

PARCHE, 13

POGY, 12

PUFFER, 12

SCULPIN, 41

SNOOK, 41

TAUTOG, 12

TUNNY, 13

WILLAM H. BATES, 12

Internuclear Ca, Missouri, University of, 9

Iowa Electric Light \& Power Co., Duane Amold

Energy Center, Unia 1,2

Iowa Sinte University, 9
Taknhama Power Station, Unit 1, 17

Tokai Na. 2 Power Stution, 17

Tsuruga Power Stution, Unit 1, 17

Japen Power Demonstrution Reactor, 44

JEFFERSON CITY, 14

JOHN ADAMS, 42

JOHN C. CALHOUN, 42

JOHN C. STENNIS, 15

JOHN MARSHALL, 42

Joseph M. Farley Nuclear Plant

Unit 1,3

Unis 23

Jose Cabren, 18

JRR-2, 20

Juggemaur, 33

K Reactor, 11

Kahl Nuclear Power Station, 44

Kaiser Engineers

Engineering Test Reactor, 31

Experimental Gas Cooled Reactor, 28

Kaman Nuclear, Fast Burst Reactor Facility, 16

KAMEHAMEHA, 12

Kansai Electric Power $\mathrm{Co}$

Mithama Power Station, Unit 1, 17

Ohi Power Station

Unit 1, 17

Unit 2, 17

Takahama Power Station, Unit 1, 17

Kansas, University of, 38

Kansas State University, 9

Kashiwazaki-Kariwa

Unit 6, 19

Unit 7,19

KE Reacior, 39

Kemknftwerk Leibstad, Switzerland, Leibstadh,

Kemknfiwerk-RWE-B yemwert, KRBI 44

Kewaunee Nuclear Power Plant, 3

KEWB, 35

KEY WEST, 13

Kinetic Experiment on Water Boilers, 35

King Intense Nextron Generetor 32

Kingles, 32

Kinki University, 23

Kiwi-A, 30

Kiwi-A Prime, 30

Kiwi-A3, 30
Laguna Vende Station

Unit 1, 17

Unit 2, 19

LAMPRE-1, 28

LAPON, 42

LAPRE-1, 28

LAPRE-2, 28

Large Ship Reactor Prototype (2 reactors), 15

Lanice Test Reactor, 40

Lawrence Livemore National Laboratory

Experimental Propulsion Test Reactor, TORY II A, 43 TORY II C. 43

Nuclear Effects Reacto

KUKLA, 35

FRAN, 35

Super KUKLA, 44

Leibstadt, 18

Leland Simfond University, 38

Lemoniz, Unit 1, 19

Lemoniz, Unit 2,19

LEWIS AND CLARK, 42

Limerick Genernting Stution

Unil 1,3

Unit 23

LTRR, 35

Livermore Pool Type Reactor, 35

Livermore Water Boiler, 35

LWWB, 35

Loctheed Aircraft Co., Critical Facility for RER, 46

Lockheed Aircraft Corp

Colombia, IAN-R1, 20

DOE Demonstration Reactor, 34

European-Asian Exhibit Program, 34

Lockheed Aircraft Corp., 35

NASA Mock-Up Reactor, 35

Ohio State University, 10

Purdue University, 10

Radiation Effects Reactor, 36

LOFT, 32

London University, Queen Mary College, United Kingdom, 45

LONG BEACH (2 reactors), 14

Long Island Lighting Co., Shoreham Nuclear

Power Station, 26

LOPO, 35

LOPRA, 9 
Los Almos Fest Reacior, 35

Los Almas LOPO Reactor, 35

Los Almos Mohen Phutonivm Reactor

Experimen, 28

Los Alamos National Laboratory

Bis Ten, 23

Comet, 23

Flunop, 23

Fuel Element Test Bed, 30

Fuel Element Test Renctor

Pewee-1, 30

Pewee-2, 30

Godiva-IV, 23

Honeycomb, 23

King Intense Neutron Generator, 32

Kiwi-Transient Test Reactor, 32

Los Alsmos Fast Reactor, 35

Los Almos LOPO Reactor, 3 F

Los Alamos Molven Pluecnium ieactor Experiment, 28

Los Alamos National Laborniony [Planned], 7

Los Alamos Power Reactor Experiment

No. 1,28

No. 228

Los Alrmos Water Boiler

HYPO, 35 SUPO, 35

Mars. 23

Nuclear Rocket Reactor Experiment

Kiwi-A Prime, 30

Kiwi-A3, 30

Kiwi-B1A, 31

Kiwi-B1B, 31

Kiwi-B4A, 31

Kiwi-B4D, 31

Kiwi-B4E, 31

Phoebus 1A, 31

Phoebus IB, 31

Phoebus 2A, 31

Omega West Reactor, 7

Parka, 46

Plinet, 23

SHEBA, 23

SKUA, 23

Uhre High Tempersture Rewctor Experiment,

Venus, 46

LOS ANGELES, 13

Loss of Fluid Test, 32

I MITSIANA 15
Masechusetts, University of, 9

Muerials Teains Reactor, 31

Mroon Construction Ca. Nuclear Engineering Test Reador, 43

MoGuire, Williem B., Nuclear Suntion

Unit 1,5

Unit 2,5

McMaster University, Canada, 22

MCZPR, 9

MEMPHIS, 13

Memphis Stute University, 38

Measllurgical Laboretory

Argonne CP-3, rebuih as CP-3', 33

Chicago Pile 1, rebuilt as CP-2, 34 Mexico

Laguna Verde Station
Unit 1,17

$$
\begin{aligned}
& \text { Unit 1, } 17 \\
& \text { Unit 2, } 19
\end{aligned}
$$

National Commission for Nuclear Energy, 21

MH-1A, 41

MIAMI, 14

MICHGAN, 13

Michigan, University of (Ford Nuclear Reactor), 9

Michigan State University, 38

Mithma Power Station, Unit 1, 17

Milm University of, 45

Millstone Nuclear Power Station

Unit 1,3

Unit 23

Unit 3,3

Minas Gernis, University of, Brazil, 22

MINNEAPOLIS-SAINT PAUL, 13

MISSISSIPPI (2 reactors), 14

Mississippi Stule University, 38

Missouri, University of, 9

Missouri a Rolla, University of, 9

MTR-II. 9

Mitsubishi

Mihama Power Station, Unit 1, 17

Ohi Power Sinion

Unit 1, 17

Unit 2, 17

Takahuma Power Sintion, Unit 1, 17

ML-1, 43

Mont 20

Mobile Low Power Plant No. 1, 43

Motificutions and Additions to Reactor Facility,

Mohen Seh Resctor Experiment, 28
POLLACK, 41

TRUXTUN (2 reactors), 14

NEWPORT NEWS, 13

Newpon News Shipbrilding \& Dy Dock Co

ABRAHAM LINCOLN (2 reactors), 14

ALBANY, 14

ARRANSAS (2 reactors), 14

ASHEVILE, 14

ATLANTA, 13

BATON ROUGE, 42

BIRMINGHAM, 13

BOISE, 14

BUFFALO, 13

CALIFORNIA (2 reaciors), 14

CARL VINSON (2 reactors), 14

CHARLOTTE, 15

CHEYENNE, 15

CHICAGO, 13

CINCINNATI, 13

DWIGHT D. EISENHOWER (2 reactors), 14

ENTERPRISE (8 reactors), 14

FINBACK, 12

GEORGE C. MARSHALL, 42

GEORGE WASHINGTON (2 reactors). 14

GEORGE WASHINGTON CARVER, 42

GREENVILLE, 15

HAMMERHEAD, 12

HAMPTON, 14

HENRY CLAY, 42

HONOLULU, 13

HOUSTON, 13

JAMES MADISON, 42

JAMES MONROE, 42

JEFFERSON CTTY, 14

JOHN C. CALHOUN, 42

JOHN C. STENNIS, 15

JOHN MARSHALI 42

KEY WEST, 13

L. MENDELL RIVERS, 13

LAPON, 42

LFWIS AND CLARK, 42

LOS ANGELES, 13

MEMPHS, 13

MISSISSIPPI (2 reactors), 14

MONTPEIIER, 14

NEWPORT NEWS, 13

NIMITZ (2 reaciors), 14

NORFOLK, 13

OKLAHOMA CTTY, 13
Unir 2,3

Unit 3,3

Northen Stetes Power Co.

Monticello Nuclear Genention Plart 3

Perhfinder Alomic Plant 25

Prisie Island Nuclem Genereting Plan Unie 1.4

Unia 24

Northrop Componte Labomaries, 35

NR-1, 14

NRAD, 8

NRF, 35

NRX-A2, 30

NRX-A3, 30

NRX-A4EST, 30

NRX-A5, 30

NRX-A6, 30

NSRR, 20

Nuclen Effects Reactor, 35

KUKLA, 35

Super KUXILA, 49

Nucterr Enginescins Teet Reactor, 43

Nuclear Examination Reactor, 36

Nuclear Rocket Engine Reactor Experiment (NERVA)

NRX-A2, 30

NRX-A3, 30

NRX-A5, 30

Nuclear Rocket Reactor Engine System Test (NERVA)

NRX-A 4EST, 30

Kiwi-A, 30

Kiwi-A Prime, 30

Kiwi-A3, 30

Kiwi-B1A, 31

Kiwi-B1B, 31

Kiwi-B4A, 31

Kiwi-BAD, 31

Kiwi-B4E, 31

Kivi-A, 30

Phoctus 1A, 31

Phoebus 1B, 31

Phoetons 2A, 31

Nuclear Safecy Fecility, Rocky Fius Plant

HorizonenlSplit Table, 24

Solution Bese, 24

Vericalsplit Table, 46 
Lonivima Sume Univerrity Nedear Science Ceneer, 35

LOUISVILLE, 13

Low Inemsity Tex Reactor, 35

Low Tempereture Neutroa Irmdimion Facility, 35

LPIR, 35

LRP, 33

LTII, 35

LTR, 40

LWBCC, 46

Lynchbers Resench Center, Critical Fecility-10, 46

Memashen

Unit 1, 19

Unit 2,19

MANE, 15

Maine Yastree Alomic Power Co. Maine Yankee Alomic Power Plone, 3

Mina, University of, Germany, 22

Malnysin, Tum Ismail Alomic Research Cestre,

Menbetuan Collese, 9

Mare Island Naval Shipyard

ANBREW JACKSON, 42

DANIEL BOONE, 42

DRUM, 12

GUTMARRO, 12

GURNARD, 12

HALBBUT, 41

HAWKBIL, 12

KAMELHAMEHA, 12

MARIANOG. VALLEJO, 12

PERMTr, 41

PINTADO, 12

PLUNGER, 41

SARGO, 41

SCAMP, 41

STONEWAL JACKSON, 12

THEODORE ROOSEVELT, 41

WOODROW WII SON 42

MARF, 15

MARIANO G. VALLEJO, 12

Mars, 23

Marin Marietra Corp.

Pontable Medium Power Plant

$$
\text { No. } 1,40
$$

Na. $3 \mathrm{~A}, 41$

STURGS Flowing Nuclear Power Plant, 41

MARYLAND, 13

Maryland, Universiny of, 9
Monticello Naclear Genercine Prang 3

MONTPEITER, 14

Morocoo, C.EN., Momiocen, 22

MSRE, 28

MTR 31

Mulleim-Kertich, 16

Monich, Technical University of, Germany, FRM Gerectines 22

MUR, 35

MURR 9

Musahi College of Tectundogy, 23

Muhleber. 18

NReactor, 26

NARWHAL, 12

NASA Mock-Up Reactor, 35

NASA-TR, 31

NATHAN HALE, 4

NATHANAEI GREENE, 42

Netiond Aeronantics and Spece Adminimaterion

Plezin Brook Reactor Freility, 31

National Instivuce of Simadard \& Technology, ?

Natural Circulation Test Plent, 15

NAUTILUS, 41

Naval Research Laboretory, Naval Research

Reaceor, 44

NEBRASKA, 13

Nebraska Public Power District, Cooper Nuclear

Station, 2

Netherlands,

Dodewanad, 17

Energy Center, 20

NETR, 43

Neutron Radiography Facility

NRAD, 8

NRF, 35

NEVADA 13

Nevada, Universiry of, 38

New Mexico, University of, 10

NEW YORX CTTY, 13

New York Power Authority

Indim Point Surion, Unit 3,3

Inmes A. Filz Parick Nuclear Power Piant; 3

New York Shipbuilding Corp.

GUARDFISH, 42

HADDO, 41

Nuclear Ship SAVANNAH, 27

POGY, 12
Ornta 13

QUEENFEYH, 42

RAY, 42

ICHARD B. RUSSEI 42

ROBERT E LEE, 41

SALT LAKE CTYY, 13

SAM HOUSTON, 42

SAM RAYBURN, 12

SAN FRANCISCO, 13

SCRANTON, 14

SEA DEVI, 42

SHARK, 41

SIMON BOLIVAR, 12

SOUTH CAROLNA (2 Teacers) 14

SPADEFFH, 12

TEXAS (2 reactor), 42

THEODORE ROOSEVELT (2 resctors), 14

THOMAS JEFFERSON, 42

TOLEDO, 15

TUCSON 15

UNITED STATES, 15

VIRGIIA (2 reactors), 14

VON STEUBEN, 42

NF-1, 30

Ningan Mohawt Power Corp.

Nine Mile Point Nuclear Station

Unit 1,3

NIMTTZ (2 reactors) 14

Nine Mile Point Nuclear Stnion

Unit 1,3

NIST, 7

Nordostschweizerische Kraftwerke AG,

Switzerdand, Beman

Unit 1, 18

Unit 2, 18

NORFOLK, 13

North Anna Power Station

Unin 1,3

Unit 23

North Atimtic Energy Service Corp. Seabrook Nuclear Station, Unit 1, 4

Nonh Carolina State Universiry

Graphine/water, 38

PULSTAR, 10

Northesst Nuctear Energy Ca.

Millstone Nuclear Power Sumion Unin 1,3
Wenerect The 46

Nocier Suip SAVANNAH, 27

Nucheer Symems Ascociutes, Misciscippi Stute

University, 38

Onk Ridge Critical Experiments Fuciliny, 4

Onk Ridge Grophiev Reactor, 36

Ont Ridge Resench Reactor, 32

Onk Ridge National Labormary

Advanced Neution Source Reactor, 8

Aircraft Reactor Experimeat, 43

Balk Strielding Reactor, 34

Healh Phyrics Research Reactor, 34

High Finx Isotope Reector, 7

Homogeneous Reactor Experiment No. 1, 28

Homogeneous Reactor Experiment No. 2, 28

Low Intensity Test Reactor, 35

Low Tempereture Neutron Imadiation Facility, 35

Miverials Testing Reactor, 31

Molven Sult Reactor Experiment, 28

Oak Ridge Research Reactor, 32

SNAP-02/10A TSF Shielding Experiment, 29

Switzentand 21

Tower Shielding Reactor, 36

Tower Shielding Reactor No. II, 36

Oconee Nuclear Stution

Unit 1,3

Unit 2,3

Unit 3,3

Ohi Power Sintion

Unit 1,17

Unit 2,17

OHO, 13

Ohio Edison Co., Duquesse Light Co.,

Beaver Valley Power Station, Unit 1,

Ohio Stute University, 10

Othahoma, University of, 38

OKLAHOMA CTTY, 13

OLYMPIA, 13

OMAHA, 13

Oraha Public Power Distric

Fon Cillhoun Station, Unit 1,2

Omahe Veterans Administration Hospitnl, 8

Omega West Reactor, 7

OMRE 29

Oregon Strte University

AGN-201-114, 38

OSTR, 10 


\section{REAcTOA nDEX (Contumied)}

Cica, 36

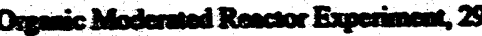

CBNL Pool Citical Aswembly, 46

osis3 3

$\cos 10$

costr, 10

OWR, ?

Oyder Croek Noclear Power Phat, Unit 1, 3

P Rowavo, 39

Pacife Cos 1 Eoduic Co.

Dinblo Crayon Neclear Power Flant, Unit 1.

Funbelit Boy Power Fland, Unit 3,25

Peific Northwe Labocesory

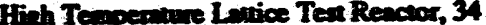

Phycienl Consts Tea Reacur, 36

Then Tee Reacor No. 2, 36

Puliem, PARR, 21

Pilesmo, Universing of, 23

Prinden Nodear Prot Unit 1,3

Puto Verde Neclear Cenerening Surion

Unit 1,3

Unit 23

Unit 3,3

PARCHE, 13

PARCO, 12

Puiton, 46

PARR, 21

PACADENA, 14

Pet.finder Alomic Fent 2

PATRFCK HENRY, 41

Puvia, University of, 23

Pawling Research Reactor, 36

PBF, 32

PCA, 46

PCT, 36

PDP. 40

Peach Bowon Alcuic Power Stution

Unit 1,25

Unit 2,3

Unin 3,3

Pens Stute TRIGA Reactor, 10

PENASYLVANL, 13

Penaryivania Power \& Light Co, Susquehanm Siem Electric Simion

Unit 1,5

Unie 2 ,

Penonglvenia Sote Univessiny, Pena State TRIGA Reacior, 10
POLACK, 41

Clynochic lanimite of New Yats, 38

Poruble Nodion Power Plan

No. 1.40

Na. 21,40

No. 31,41

Puthad Electic Co, Trojon Nuctear Plact, Unis 1, 26

PCRTSMOUTH, 13

Pontomouth Nowel Shipgend

GRAYLNG, 12

JACK, 41

JOMN ADAMS, 42

NATHANAEL GREENE 42

SAND LANCE, 12

SEADRAGON, 41

SWORDFSH, 41

THRESHER, 41

TiNos, 42

Pormon, RP-1. 21

Power Reactor Dovelopmene Compeny, Enrico

Feni Avomic Power Plont, Unit 1.25

Power-Bean Fecility, 32

Privie Illand Noclem Genereing Plant Unit 1, 4

Unit 2,4

Process Development Pite, 40

PROVIDENCE, 13

PRR, 36

PRR-1, 21

PRTR, 29

PSTR, 10

PTF, 46

PTR 45

Public Service Co. of Colorudo, Fon SL Vrain Nucben Genersuing Sution, 25

Public Service Electric \& Gas Co

Hope Creek Nuclear Genernting Simion Unin 1.2

Salem Nuctear Generning Sution

Unil 1,4

Unit 24

Prento Rico Nuclear Censer

$L-7,38$

TRIGA-FLP, 38

Prenso Rico Water Resources Avthority, Boilin

Nuclear Superhener Power Simion, 25

PUFFER, 12

PUISTAR
Expesinent Orgmic Cocled Rences, 2

Germery, Brom BoverilKropp, 45

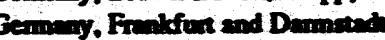
Univenities of, 45

Hellon Nuclear Power Fecility, Sheldon

$$
\text { Simion, } 25
$$

Mincis Instiune of Techaolony Reacanch

$$
\text { Intinese, 35, } 45
$$

Kinetic Experineme on Water Boilen, 35

Livemore Wree Boiler, 35

Neved Univering of 38

Nuclear Exemination Renctor, 36

Onzmic Modereted Renctor Experineses, 20

Piqua Nuclear Pomer Fecility, 26

Preso Rion Nuclear Certer, 38

Rockwell Intemenional, 36

Shield Tea and Irradietion: Reactor, 36

SNAP-Q2 Developuental Symeen, 29

SNAP-C2 Experimental Reacer, 29

SNAP-08 Developmentel Reaceor, 29

SNAP-OS Experiunemel Reactor, 29

SNAP-1OA Flight Sytem Ground Test Na 1

SNAP-10A Finghe System Ground Test Na. 3.

SNAP-10A Flight System, S10FS-4, 29

SNAP-10A Flighe System, S10FS-5, 29

SNAP-10A Trensient Tea No. 2, 32

SNAP-10A Trmesient Tear No. 3, 33

SNAP-02nOA TSF Shielding Experiment, 29

Sodivem Reactor Experimenc, 29

Walter Reed Research Renctor, 44

West Bertin, City of, 45

Wyoming Universiny of 39

Rockwell Intemationil, Rhode Idland Nuclear

$$
\text { Science Center, } 8
$$

Romaria

TRIGA (MPR 16), 20

TRIGA-ACPR, 20

RP-1,2

RRR, 38

RSTM, 46

Rural Coopernive Power Associntion, Ekt River Reactor, 25

SiC, 43

SIG, 43

S2DS, 29

S3G, 43
SEAWOLF, 15

SEAWOLF PWR 4

SEAwOL F Sotimen Reacor, 41

SEROR, 29

Ceibeadof Resench Center, Amorie, Atth, 20

Sequoysh Nedear Plane

Unit 1.4

Uni: 24

SER, Sendia Engineerieg Reactor, 32

SER, SNAP-12 Experimental Reador, 29

$\sin 14$

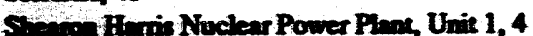

SHEBA, 2

S.icid Teat and Irradintion Reactor, 36

Shippungpeat Alomic Power Stution, 26

Shorehno Nodeer Power Staican, 26

MVVESTDES, 12

STMON BOUVAR, 12

SRATE 41

SKIPJACK, 41

SKUA, 23

SL-1, 43

Slovenia, Joeef Svefm Nuclear Inximue, 21

Slovenia, Krsko, 18

$S M-1,41$

SM-1A, 41

Small Submarine Reactor Prototype, 43

SNAP-O2 Developmentil System, 29

SNAP-02 Experimental Reactor, 29

SNAP-02nOA TSF Shielding Experiment 29

SNAP-08 Developmental Reactor, 29

SNAP-08 Experimental Reactor, 29

SNAP-10A Fight System

S10FS-4, 29

S10P5 $-5,20$

SNAP-10A Fight System Ground Test No. 1,29

No. 3,29

SNAP-10A Tranient Test

No. 2,32

No. 3,33

SNAP-TSF, 29

SNAPTRAN-2, 32

SNAPTRAN-3, 33

SNARE, 35

SNL Ammolar Core Research Reactor, 8

SNL Critionl Assembly, 23

SNOOK, 41

SNRS-1, 16 
PERMT, 41

Peny Ninclear Power Plea

Uni 1,4

Unir 2,5

Pewiet-1,30

Pewee-2, 30

PHILADEI PFIK, 13

Phitedelphin Electric Co.

Limerick Generning Simion

Unit 1,3

Unit 2, 3

Peach Bonom Alonic Power Suntion

Unit 1,25

Unit 2,3

Unit 3, 3

Philippines, Republic of the

Philippine Neclear Power PImat, Unin 1, 19 PRR-1, 21

Phillips Petrolean Ca

Nuclear Effects Reacroc, 35

SNAP-10A Transient Tea No. 3, 33

Special Power Excursion Renctor

Ten Na. 1, 33

Teat Na. 2, 33

\section{Phoebos 1A, 31}

Phoebus 1B, 31

Phocten 2A, 31

PHOENDX, 13

PHRENIC, 34

Physical Cosstmest Tex Reactor, 36

Pilgrim Nuclear Power Sintion, Uhit 1, 4

PINTADO, 12

Piqm, Ciry of, Piqua Nuclear Power Fecility, 26

Piqua Nuclear Power Facility, 26

PITTSBURGH, 13

Pinnet, 23

Plum Brock Reactor Facility, 31

PLUNGER, 41

Phucaium Recycle Test Renctor, 29

PM-1, 40

PM-3A 41

PNL Critical Mass Laboratory

FEAS, 46

Horizonal, 46

RSTM, 46

POGY, 12

Point Beach Nuclear Plan

Unit 1,4

Unie 2.4

New Yock, Sune Universiny of, 10

North Carolim Sume Universiny, 10

PUR-1, 10

Purdue Universiny, 10

Unin 1,4

Unir 2, 4

QUEENFSH, 42

R Reactor, 39

Radiacion Effects Reactor, 36

Radiation Research, Association for, Germany.

Rancho Seco Nuclear Generuting Sention, Unit 1

$$
26
$$

RAY, 42

Reed College, 10

Regional Center for Nuclear Studies, Zrire, 22

Rensselaer Polyrectnic Inatime, Troy, NY, 23

RER, 36

Rheinesch-Westfelisches Elektrizitnetswerk AG, Germany, Mulheim-Kretich, 16

Rhine-Westphalia Power Co., RWE, Germany,

Kahl Nuclear Power Sintion, 44

RHODE ISLAND, 15

RICHARD B. RUSSEL, 42

Rikkyo University, 23

Ringhals

Unit 2, 18

Unit 3, 18

Unit 4, 18

RINSC, 8

Riso National Laboratory

Denmank

DR-1, 20

$$
\text { DR-2, } 45
$$

River Bend Stution, Unir 1,4

ROBERT E. LEE, 41

Roben Emmen Ginna Nuclear Power Plane,

Unit 1,4

Robinson, H.B., Plant, Unir 2, 2

Rochester Gas \& Electric Corp., Robent Emment Ginna Nuclear Power Plane, Unit 1,4

Rockwell Intemational, 36

Rockwell Imemntional, A

Alomics International, 33

Brigham Young University, 3

Califomia, Santa Barbara, University of, 37

Denmark, DR-1, 20
SSG, 15

SSW, 44

SSW for HMS DREADNOUGHT, 44

S8DR, 29

SBER, 29

SeG, 1

S1OFS-1, 29

S1OFS-3, 29

S10FS-4, 29

S10PS-5, 29

Secremento Municipdl Utility Districa, Rencho

Seco Naclear Generating Station, Unil 1, 26

Sefari-1, 20

Salem Nuclear Genernting Station

Unit 1,4

Unit 24

SALT LAKE CTTY, 13

SAM HOUSTON, 42

SAM RAYBURN, 12

SAN FRANCISCO, 13

SAN JUAN, 14

Sen Onofre Nuclear Genernting Strion

Unit 1,26

Unit 2,4

Unit 3,4

SAND LANCE, 12

Sendia Engineering Reactor, 32

Sandia Nationel L tomeries

Louisiana Sinte University Nuctear Science Cemer, 35

Sandia Engineering Reacior, 32

Sandia Pulsed Reactor II. 8

Sendia Prined Reaceor III 8

SNL Amnular Core Research Reactor, 8

SANTA FE, 14

Senta Maria de Garoan, 18

Soo Paulo, University of, Brail, 22

SARGO, 41

Saxton Nuctear Experimental Reactor Project, 29

SCAMP, 41

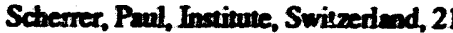

SCHTro, 34

SCORPION, 41

SCRANTON, 1

SCULPD, $4 !$

SEA DEVIL, 42

SEA HORSE, 12

Seabrock Nuclear Station, Uni 1,4

SEADRAGON, 41
Sodven Rencer Experinent 20

Solution Base, 24

Souch Africa, Safmi-1, 20

SOUTH CAROLNA (2 reactors), 14

South Carotim Electic \& Ges Co

Vingil C. Summer Nuclear Semion, Unit 1,5

Soun Teras Project

Unit 1,4

Socthem Crifomin Ediec

Sen Onofic Nuctear Genemting Sinio

Unil 1,26

Unit 24

Unit 3,4

Southem Nuclear Opernting CO, Joseph M. Failey Nuclear Pinat

Southwea Experimental Faxt Oxide Reaceor, 29

SPSE, 40

SPADEFSH, 12

Spain

Almane

Unit 1, 18

Unit 2, 18

Ascos

Uni 1,18

Unit 2, 18

Cofremes

Unin 1, 18

Jose Cabreto, 18

Lemoniz

Unit 1, 19

Unir 2, 19

Nuclear Energy Bond-JEN, 21

Sinta Maria de Garome, 18

Valdecaballeros

Unit 1, 19

Unis 2,19

Vandellow

Unit 2,18

Special Power Excursion Reactor Tes

No. 1.33

No. 2, 33

$\mathrm{Na} 3,33$

No. 4,33

SPERT-1,33

SPERT-2, 33

SPERT-3, 33 


\section{REACTOR WDEX (Continued)}

SFERT-4,33

SPR, 36

SPR-II, 8

SPRNGFIELD, 14

SR 305-M Teat Pile, 40

SRE, 29

St. Lucie Plant

Unit 1,4

Unit 24

Si. Vrin, For, Nuclear Generning Stution, 25

Sundand Pile/Subcritical Experimental Complex,

40

State Universing of New Yotk, 10

Suticnary Low Power Pland No. 1, 43

Stutionary Medium Power Plant

No. 1,41

No. 1A, 41

Stationary Neurron Radiogmphy Sysem, 16

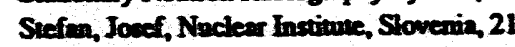

STIR, 36

STONEWALLJACKSON, 12

Sudsvik Enorritelerrik, Sweden, 20

STURGEON, 12

¿ STURGIS Fouting Neclear Power Pling, 41

Submarine A duanced Reactor Prototype, 43

Submarine Intermediete Reactor Mart A, 43

Submarine Thermal Reactor Fecility, 43

Summer, Vizgil C., Nuclear Sution, Unit 1, 5

SUNFISH, 12

Super KUKIA, 44

SUPO, 35

Surry Power Sintion

Unil 1.5

Unit 2.5

Susquehanna Steam Electric Stution

Unit 1.5

Uniz 2,5

Sweden, Ringhals

Unit 2, 18

Unit 3, 18

Unit 4, 18

Sweden, Sewdsvik Energinctonit, 20

Switzertand

Basel, University of, 23

Bernan

Unir 1, 18

Unin 2,18

Geneva, University of, 45

Leibstach, 18
TRIGA-Mar II, 10

Texas Utulhies Generting Co., Comanche Peat Sven Electric Station

Unit 1,2

Unir 2,2

Thailand, TRR-1, 21

THEODORE ROOSEVELT, 41

THEODORE ROOSEVELT (2 rescans), 14

Thermal Tea Reactor

No. 1,44

No. 2,36

THOMAS A. EDISON, 42

THOMAS JEFFERSON, 42

Three Mile Istand Nuclear Sution

Unit 1,5

Unit 2,26

THRESHER, 41

Thenge

Unit 1.16

Unit 3,16

TINOSA, 42

Tokri Na. 2 Power Strion, 17

Tokyo Electric Power Co.

Fukushima Dai-ichi Power Station

$$
\text { Unit 1. } 17
$$

Unit 2, 17

Unit 6, 17

Kashiwanki-Kariwe

Unit 6, 19 Unit 7,19

TOLEDO, 15

Toledo Edison Co., Devis-Besse Nuclear Ponver Station, Unit 1.2

TOPEKA, 14

Tonrey Pines, TRIGA-M III Reacaor, 36

TORY IIA, 43

TORY IC, 43

Toshibe

Fukushime Dai-ichi Power Suntion Unin 2, 17 Unit 6, 17

Kashiwazaki-Kariwa Unil 6, 19

Tower Shielding Rewaror, 36

Tower Shielding Reactor Na II, 36

Transient Reactor Tex, 7

TREAT, 7

TREPANG, 12
TRIGA-Min, 10

UTR Tear Reactor, 36

UTR-1, 33

UTR-10

lown Sime University, 9

Virginin Pobrochmic Lativese 39

UVAR, 10

Valdecabellexu

Unie 1, 19

Unit 2, 19

Vallecitos Boiling Water Rencer, 29

Vendelloce, Unie 2, 18

Vawenfill AB, Sweden, Ringhuls

Unie 2.18

Unit 3,18

Unit 4,18

VBWR, 29

Venemela, hative for Scientific Recench, 22

Venen, 46

Vermos Y unbee Noclear Power Corp, Vemont Yankee Neclear Power Stution, 5

Vericalveplie Trables 46

Vienm Pobyoctaic Intiomer, Ansuin, 22

Vietuan, Viennen Inatime of Nuclear Research, 45

Virgil C. Sumener Noclear Station, Unit 1.5

VRCINIA (2 reactoon). 14

Virginia, University of

CAYALIER, 39

UVAR, 10

Virginin Electric \& Power Co.

North Arma Poner Suntion

Unit 1.3

Surny Power Simica

Unit 1,5

Unin 2,5

Virginia Polynechaic linatituse, 39

Vitro Corpontion of Americen, Comell Universiny

Zeso Power Reacect, 9

Voptle, Alvin W., Noclear Plane

Unit 1. 1

Unit 2,1

VON STEUBEN, 42

Waher Reed Arny Instivuce of Resenuch, Waber Reed Research Reacior, 44

Wethinglos, Univerning of, 39

Weahinetan Nuclear Project

Unit 1.5

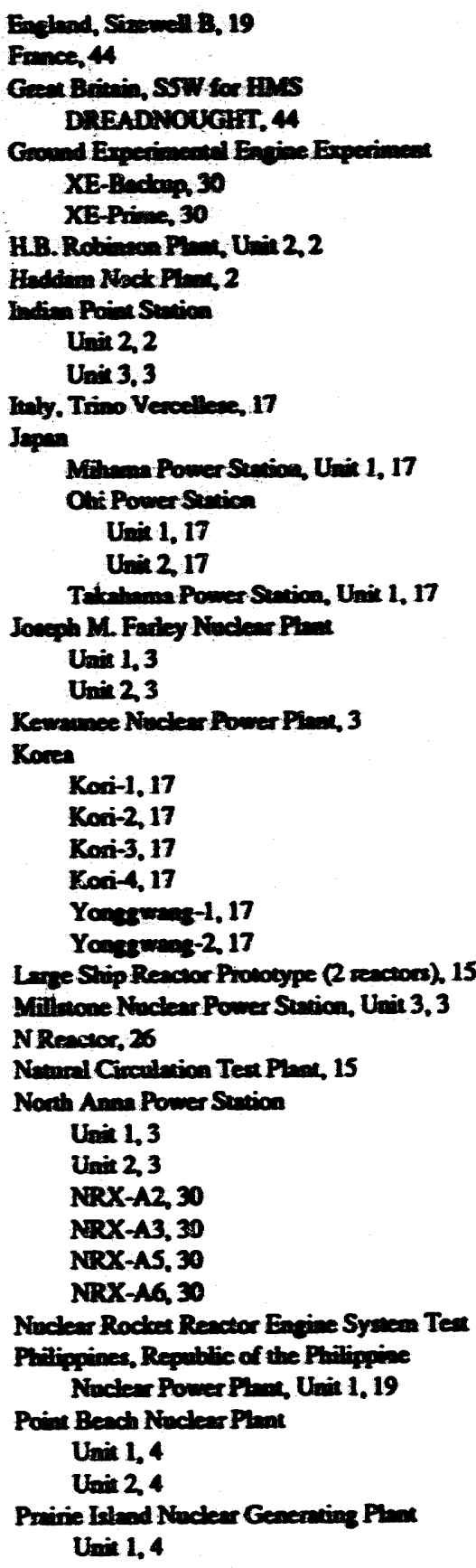


Nalebers, 18

Puil Scherver Inoimen 21 SWORDESH, 41

\begin{tabular}{|c|c|}
\hline Trime & $x_{0}$ \\
\hline & $\begin{array}{l}U_{\min 1,}^{18} \\
u_{\text {nit }} 2,18\end{array}$ \\
\hline$K=0$ & Shem 19 \\
\hline & Unit 2, 19 \\
\hline Lone & men \\
\hline & $\begin{array}{l}\text { Unit 7, } 19 \\
\text { Uni } 8,19\end{array}$ \\
\hline Meax & nethen \\
\hline & $\begin{array}{l}U \min 1,19 \\
U \min 2119\end{array}$ \\
\hline
\end{tabular}

Trkahuma Power Sunion, Unis 1, 17

Tarpour Nuclear Power Sunion

Unit 1, 17

Unit 2, 17

TAUTOG, 12

TECUNMSER, 42

Tehrem, University of, Iren, 23

TENRESSEE, 13

Tenneseee Valley Ambority

Bellefoute Nractear Ptant

Unit 1,5

Unie 2,5

Browns Fenty Nuciear Power Strion

Unit 1, 1

Unit 2, 1

Unit 3.1

Sequogath Noclear Plent

Unit 1,4

Unit 2, 4

Watus Ber Noclear Plent

Unim 1.5

TEXAS (2 reactor), 42

Teras

Sonth Teran Projed

Unit 1.4

Unit 2,4

Texas AdM Univeriily

AGN-201M-106, 10

TRIGA, 10

Texas a Ausin, University of,

TRIGA-MK I. 39
Tideat Prowype, 15

Trino Vercellese, 17

TRTON (2 Reacion), 41

Trojen Nuclear Plex, Unin 1, 2

IRR-1, 21

TRUXTUN (2 reatoon) 14

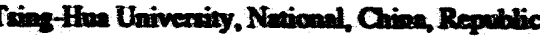
of, 22

TSR, 36

TSR-2, 36

Taurnge Power Stution, Unit 1, 17

TTR-1, 44

TIR-2,36

TUCSON, 15

TULUIBEE, 41

Tm Imail Alomic Reseanch Centre, Malaysia.

TUNNY, 13

Tuatey

Alomic Energy Comminsion, 21

Intubel, Technical University of, 21

Turkey Point Pline

Unit 3,5

Unit 4,5

Tuskegee Institute, 39

UFTR, 9

UHTREX, 29

Ulchin 3,19

Ulchin 4, 19

ULR, 9

Ulum High Temperiune Reactor Experimeon, 29

ULYSSES S. GRANT, 42

UMR-R, 9

Union Electric Co, Calloway Plone Uni 1 ,

Union Electrica, S.A, Spain, Almanaz.

Unit 1,18

Unit 2, 18

Jose Cabren, 18

United Kingdom, Queen Mary College, London University, 45

Unined Nuclear Corpontion

Anny Pulse Redimion Facility, 16

Developinent Division, 46

Pawling Reseanch Reactor, 36

UNITED STATES, 15

Unth, Univexiity of

AGN-201-107, 39
Uain 25

Unie 3,5

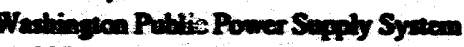
N Reacour, 26

Welingen Nodien Projed

$$
\text { Unie 1,5 }
$$

Unie 25

Unit 3,5

Wadhingen Sare Univewity, 11

Wher Reflector Tmat, 46

Wrets Bar Nocleas Plon

Unit 1,5

Unien 25

Weat Bexlin, City of, 45

WEST VROTNA, 13

Wen Vimini Univexing, 39

Westinghane Electric Coopontion

Alvin W. Vegale Nuclear Flan Unin 1.1

Unit 2,1

Beaver Valloy Power Sintion

Unis 1,

Unia 2, 1

Bnidwood Sumion

Unit 1.

Unie 2, 1

Banit, Angen 1, Centenl Electricia de Fumas 16

Byron Semian

Unit 1, 1

Unie 21

Collowny P Unit 1,

Curclines-Vinginia Tube Reactor, 25

Conmba Nuclem Station.

Unit 1, 1

Unit 22

Comenche Penk Stem Electric Stution Uni 1,2

Unit 2,2

Dirbb Canyon Neclear Power Plane

Unit 1.2

Doel

Unit 1, 16

Unit 2,16

Unit 4, 16

Donild C. Cook Nuclear Power Phan

Unin 1.2

Unit 2,2
Unis 24

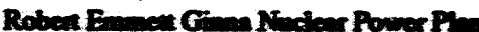

Unin 1.4

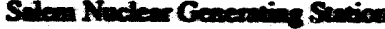

Unin 1,4

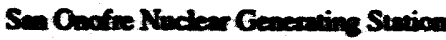
Unit 1.26

Servon Nuclear Expeninenell Reaceor Project,

Sechroat Noclear Sumion, Unit 1.4

Sequoyah Nudear Pina

Unit 1,4

Unin 2

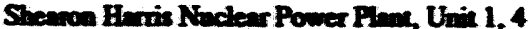

Shippinpont Atomic Power Sintion, 26

Slovenit, Rriko, 18

South Terex Projoe

Unit 1.4

Uni 2.4

Spen

Almanos

Unie 1,18

Unin 2, 18

Asco

Unie 1. 18

Unit 2, 18

Jose Cabreen, 18

Lemonix

Unit 1, 19

Uni 2, 19

Vandellos, Unit 2, 18

Submaine Thermal Reactor Facility, 43

Sumy Power Sumion

Unin 1.5

Unin 2,5

Swoden

Ringhal:

Unix 2, 18

Unit 3,18

Unit 4, 18

Switzeriond

Beanien

Unit 1, 18

Triwa

Unin 2, 18

Manestran

Unin 1, 19

Unit 2, 19 


\section{REAGTOR MDEX (Comumued)}

Theme

Un: 1, 16

Uni 3, 16

Troje Niclear Plan, Unil 1,26

They Poine PI

Un: 3,5

Unit 4,5

Vingl C. Smer Nuclear Suction, Unie 1,5

When Bar Nolear Plan

Unic 1,5

Unie 25

Wesingluane Nuclener Trinim, Center, 36

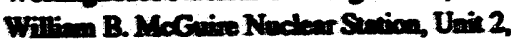

Worf Cuek Generuing Surion, 5

Yankee Nuclear Power Stmion, 20

Tion Neclear Plan

Uni 1,5

Unie 25
Wexinghome If wofoed Ca

Fen Fun Tea Fuciting, 31

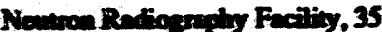

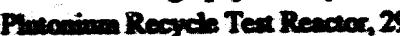

Weatingluene Nucloer Triming Cester, 36

Weringhomse Texine Reactor, 31

WHALE, 12

WIL ROCER, 12

Willin B. MoGuire Nocleer Sutio

Unit 1,5

Unit 2,5

WIULMH. BATES, 12

Willien March Rice Univeniny, 39

Wiscondin, Univernity of, 11

Wisconain Electric Power Ca,

Poine Beach Noclear Pline

Unit 1,4

Unie 2,4

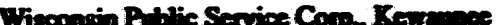
Nucicar Power Ption, 3

WNIR, 36

Wolf Croek Generating Srmion, S

Wolf Creek Nodear Operning Corp.

Wolf Creck Geverwing Stution, 5

woODROW WISON, 42

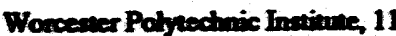

WRRR, 4

WSTR, 11

WTR, 31

WYOMMNG, 15

Wyoming Universiny of, 39

XE-Badtop, 30

XE-Prime, 30

Yankee Aromic Electric Ca, Yankee Nuclear Power Strition, 26
Yombe Nedeor Power Sunion 26

Yoneswane-1, 17

Yanewane-2,17

Yon: 2 wane-3, 19

Yonsenese-4, 19

Znire, 22

Zon Nuclear Pren

Uni 1.5

Unit 2,5

ZFFR, 23

ZPR, 9

ZPR-6, 46

ZPR-9, 16 

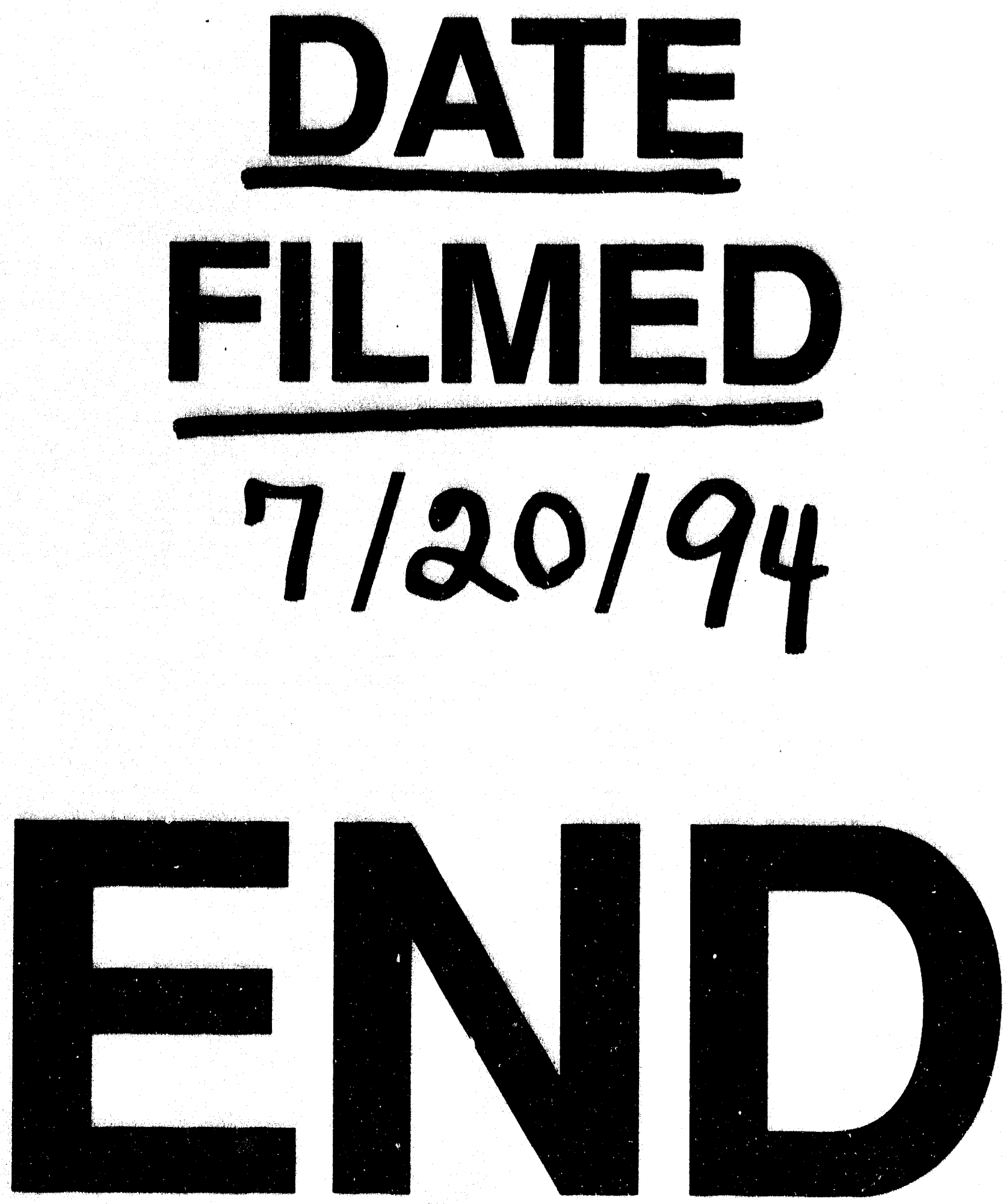
